\title{
UNCONVENTIONAL \\ ELECTROCHEMISTRY \\ IN NANOGAP \\ TRANSDUCERS
}

SAHANA SARKAR 


\section{UNCONVENTIONAL ELECTROCHEMISTRY IN NANOGAP TRANSDUCERS}




\section{The graduation committee consists of:}

\section{Chairman and Secretary}

prof.dr.ir. J.W.M. Hilgenkamp University of Twente, the Netherlands

\section{Promotor}

prof.dr.ir. S.J.G. Lemay

University of Twente, the Netherlands

\section{Members}

prof.dr.ir. J. Huskens

Universiteit Twente, the Netherlands

prof.dr. J.G.E. Gardeniers

Universiteit Twente, the Netherlands

prof. dr. A. Ewing

University of Gothenburg, Sweden

dr. K.H. Mathwig

Rijksuniversiteit Groningen, the Netherlands

dr.ir W. Olthuis

Universiteit Twente, the Netherlands

This research was financially supported by the National Institutes of Health (NIH, USA) and was carried out in the Nanoionics group, MESA+ Institute for Nanotechnology, Faculty of Science and Technology, University of Twente, The Netherlands.

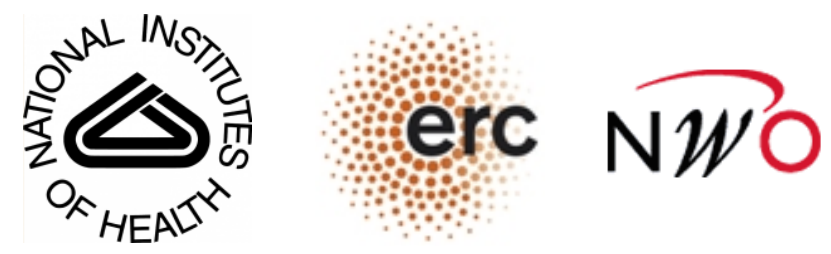

Title: Unconventional electrochemistry in nanogap transducers Author: Sahana Sarkar

ISBN: 978-90-365-4241-8

DOI: $10.3990 / 1.9789036542418$

The cover of this thesis was designed by Zinaida Kostiuchenko and Sahana Sarkar 


\title{
UNCONVENTIONAL ELECTROCHEMISTRY IN NANOGAP TRANSDUCERS
}

\author{
DISSERTATION \\ to obtain \\ the degree of doctor at the University of Twente, \\ on the authority of the rector magnificus \\ Prof. Dr. H. Brinksma \\ on account of the decision of the graduation committee, \\ to be publicly defended \\ on Wednesday 2 November 2016 at 14.45
}

by

Sahana Sarkar

born on 3 August 1987

at Purulia, India 
This dissertation is approved by :

Prof. Dr. S. G. Lemay (Promotor) 
"DNA neither cares nor knows. DNA just is. And we dance to its music."

- Richard Dawkins 



\section{Contents}

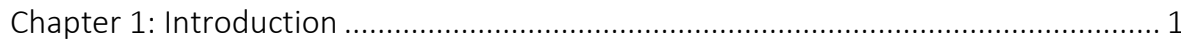

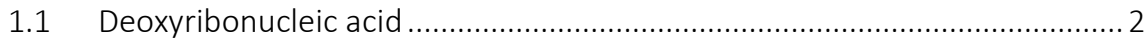

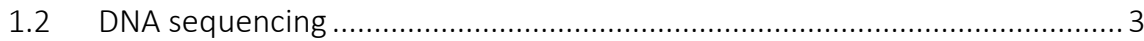

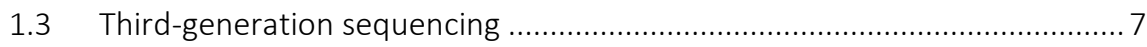

1.4 Single molecule real-time electronic sequencing .................................... 9

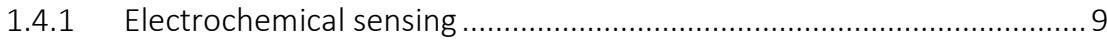

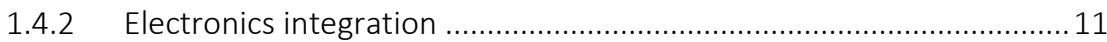

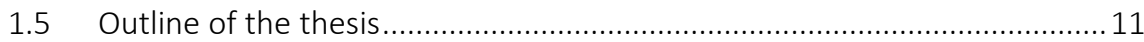

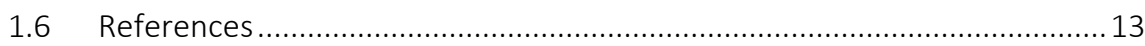

Chapter 2: Unconventional electrochemistry in micro/nanofluidic systems ........... 17

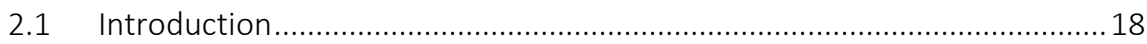

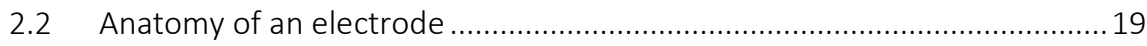

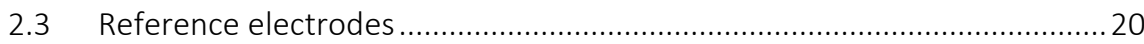

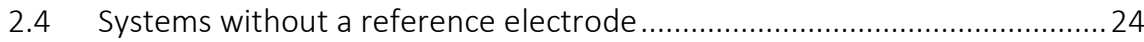

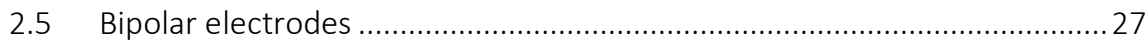

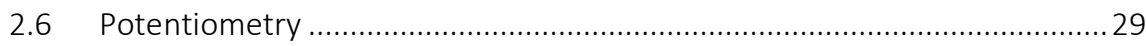

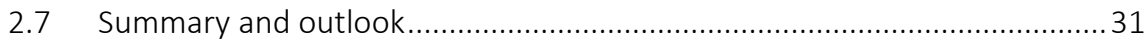

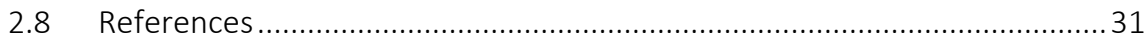

Chapter 3: Redox cycling without reference electrode ...................................... 37

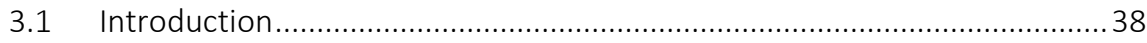




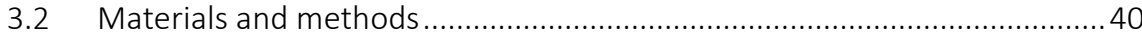

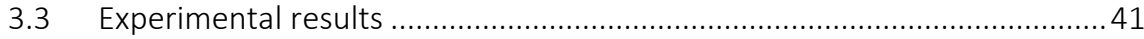

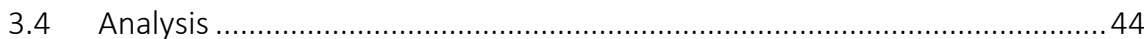

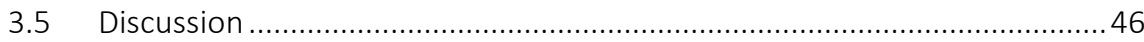

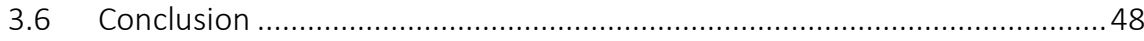

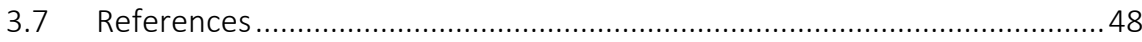

Chapter 4: Potentiometry at nanogaps for ultra-low concentration detection .........51

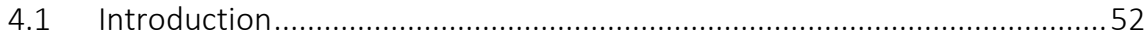

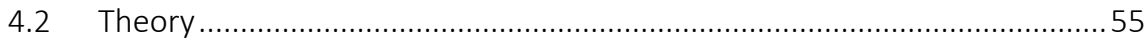

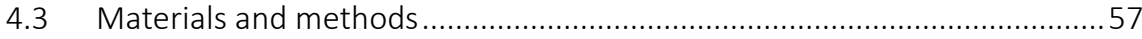

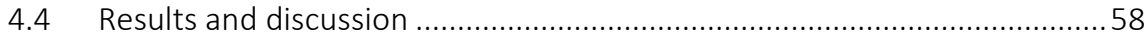

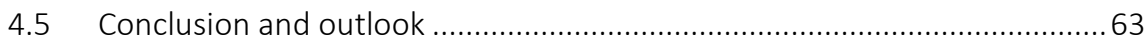

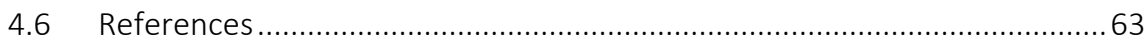

Chapter 5: Integrated glass microfluidics with electrochemical nanogap sensors ... 67

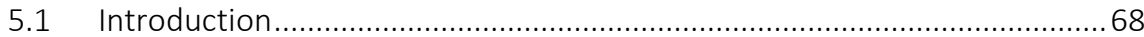

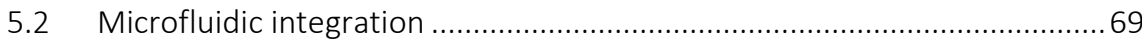

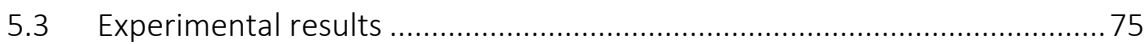

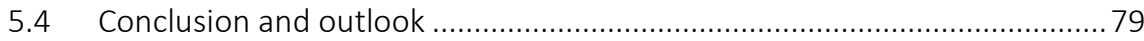

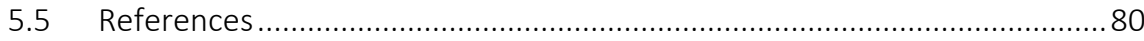

Chapter 6: Electron transfer mediated by surface-tethered redox groups in nanogaps

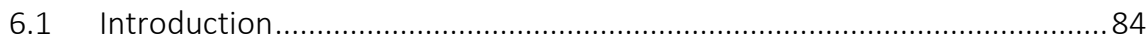

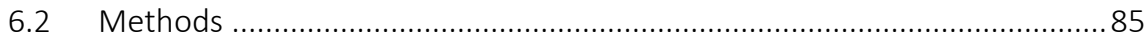

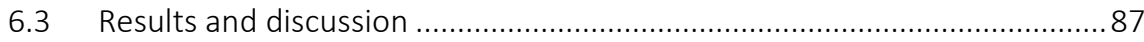

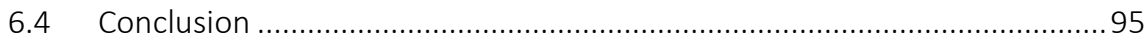

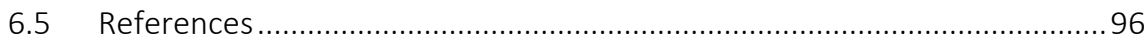




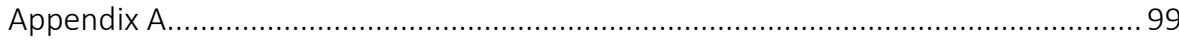

A.1 Fabrication of nanogap electrodes ……................................................ 99

A.2 Characterization of solution after exposure to device................................99

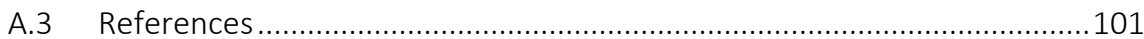

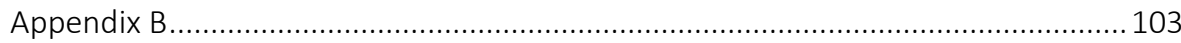

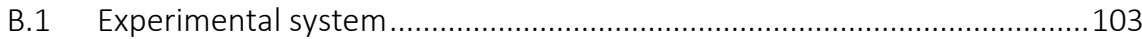

B.2 Estimation of the electrode capacitance ...............................................104

B.4 Determination of root-mean-square potential ........................................ 105

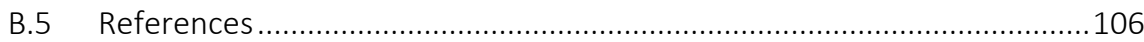

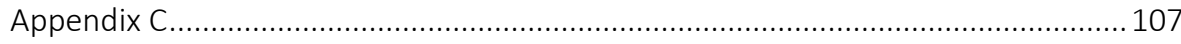

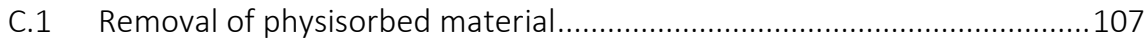

C.2 Assessment of electrode surface roughness ............................................108

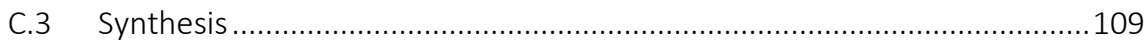

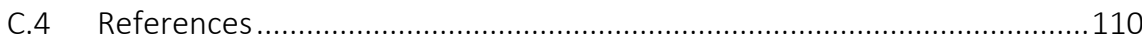

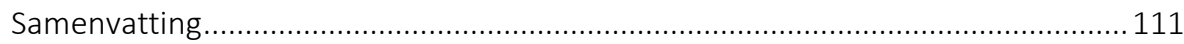

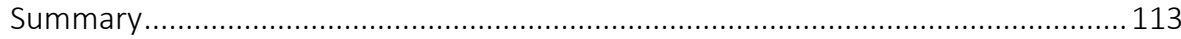

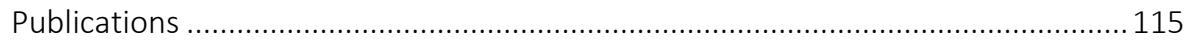

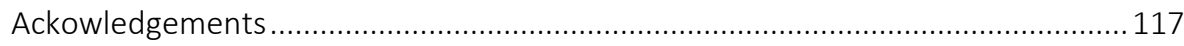

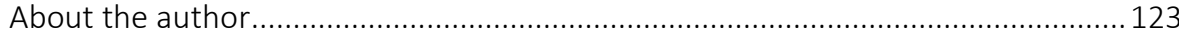





\section{Introduction}

This chapter provides a general introduction to the field of DNA sequencing techniques. It briefly describes the present-day detection methods, their challenges and future outlook on sequencing methods. A third-generation DNA sequencing technique based on electrochemical sensors, is presented that provides the overall motivation of the thesis. 


\subsection{Deoxyribonucleic acid}

In 1953, Watson and Crick solved the three-dimensional structure of Deoxyribonucleic acid (DNA) as consisting of a long chain of sugar and phosphate to which nucleotides are attached as side rungs. ${ }^{1}$ A DNA molecule consists of two strands that wind around each other like a twisted ladder (Figure 1.1a). The two strands are held together by bonds between the bases, adenine (A) forming a base pair with thymine $(T)$, and cytosine (C) forming a base pair with guanine $(G)$. The order in which the nucleotides (or bases) are arranged - their sequence-constitutes the biological information that defines every living organism in the form of a long onedimensional digital code.

(a)

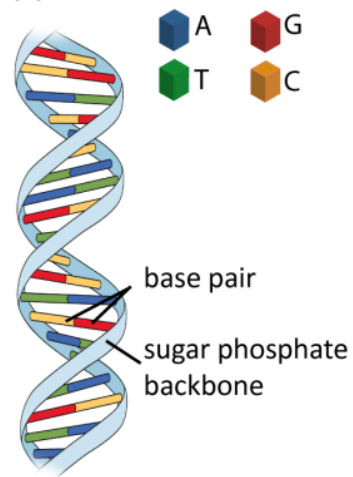

(b)

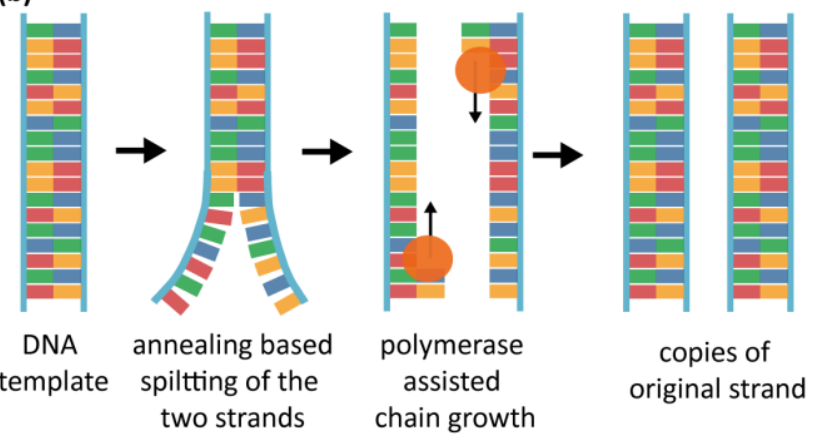

Figure 1.1. (a) Schematic of double helical structure of Deoxyribonucleic acid (DNA) consisting of a long chain of phosphate and sugar that are wound around each other due to base pair bonding. (b) Schematic of the various steps involved in polymerase chain reaction (PCR) for the amplification of DNA. Initially DNA is split into two strands. Thereafter an enzyme assisted reaction leads to the growth of the complementary strands in the presence of excess nucleotides, giving rise to two copies of the DNA.

In order to analyze DNA, it must usually be amplified, i.e. replicated into several copies. As illustrated in Figure 1.1b, this involves "unzipping" the molecule and splitting the two individual strands so that they can act as templates for new copies. In the presence of excess nucleotides, these individual strands can be grown via an enzyme-assisted reaction to form two DNA molecules consisting of one new and one old chain of nucleotides wound into a double helix once again. This forms the basic principle of the so-called polymerase chain reaction (PCR) that is used to amplify DNA and is a recurring theme in this chapter. 
(i)

input DNA

GGACGTAAGGACATCACATTCACGATCATT GGACGTAAGGACATCACATTCACGATCATT GGACGTAAGGACATCACATTCACGATCATT (ii)

$\begin{array}{llr}\text { GGACGTAA GGACATCAC ATTCACGATCAT } \\ \text { GGACGTAAGGACA TCACATTCAC } & \text { GATCAT } \\ \text { GGAC GTAAGGACATCACATTCA } & \text { CGATCAT }\end{array}$

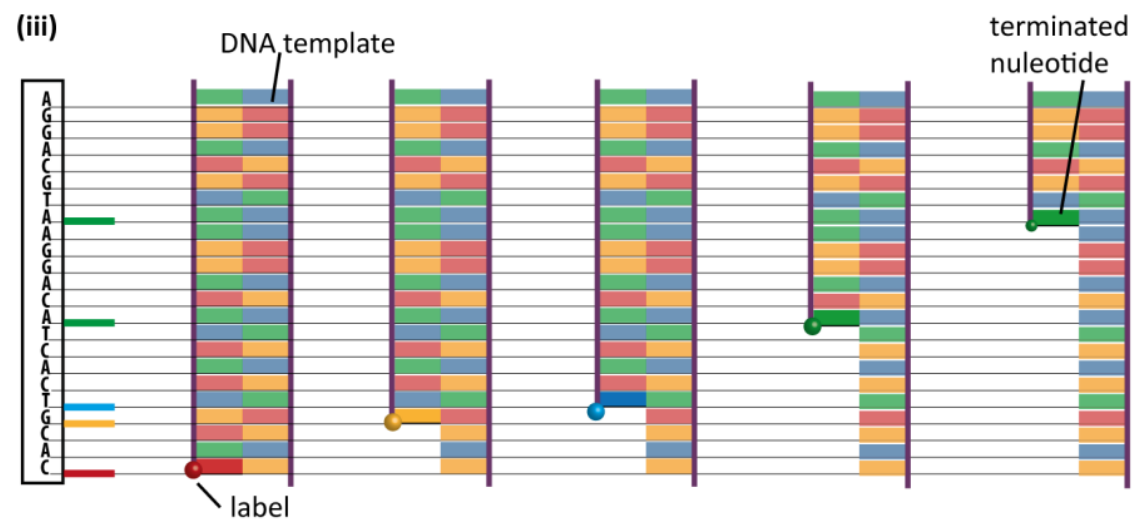

Figure 1.2. (i) Example of the sequence of an input DNA. (ii) The input DNA is split into several strands of various lengths. (iii) Schematic diagram illustrating a modern implementation of the classic first generation sequencing technique that shows the differential labelling and the use of terminators for the termination at various chain lengths. The labelled terminated ends reveal the specific nucleotides and their position in the sequence. The sequence of the strand can be determined by combining the information from the identical strands that are terminated at different locations. Image reproduced from Schadt et al. ${ }^{3}$

\subsection{DNA sequencing}

In 1977, Sanger's discovery of chain termination led to a breakthrough and marked the beginning of DNA sequencing. ${ }^{2}$ In brief, the method comprises three steps and its modern implementation is described in Figure 1.2. The first step, sample preparation, involves slicing multiple copies of the target DNA into smaller fragments. These individual fragments, which consist of a mixture of different lengths wherein the same location on the DNA appears multiple times, are once again amplified by making multiple copies. In the second step, the fragments are transferred to a solution containing, on the one hand, nucleotides and the associated enzymes, enabling chain growth, and, on the other hand, modified nucleotides with terminated ends, which serve to halt the growth upon incorporation into the growing double- 
stranded DNA. The point of termination is labelled (eg. fluorescent) which is base specific. Thereafter, all of the fragments are sorted in the order of their length using gel electrophoresis. Each different length allows for the identification of one base, namely, the terminal one, since that base is marked by the terminator label. Once the sequences of the strands are determined, the overall sequence can be reconstructed using the overlapping regions from different fragments. This was the basic principle of the first-generation sequencing (FGS) techniques. Due to its high accuracy and simplicity, the Sanger method provided the mainstay of DNA sequencing for decades.

By the 1980's, semi-automated platforms incorporating robotics, advanced imaging technologies and computer analysis programs surfaced for the purpose of improving throughputs. In 1986, Applied Biosystems released the 370A, its first commercially available four-color fluorescence automated DNA sequencer. Although fully automated, it was a slow process and allowed the sequencing of only a few hundred nucleotides at a time. In the 1990's, the Human Genome Project fueled further research towards the development of fully automated sequencing technology. The first complete human genome was sequenced in 2003. ${ }^{3-7}$ While successful, methods were still limited to read lengths of about 800 bases at a time; the project took about 10 years to complete and cost 3 billion US Dollars. ${ }^{3-7}$ These high costs and low

throughput motivated a turning point in the field of DNA sequencing and led to the development of the so-called "second-generation sequencing" (SGS) techniques.

By 2005, several SGS techniques were developed ${ }^{3-7}$ and a variety of modifications were made to the classic method of detection. A common theme of SGS techniques was achieving higher throughput by massive parallelization (reading multiple strands at a time). During sampling, arrays (or libraries) of DNA fragments were used for this purpose. Advanced detection techniques ${ }^{8-10}$ (such as pyrosequencing) were introduced and corresponding readout mechanisms were developed. Figure 1.3a sketches a "wash-and-scan" mechanism illustrating the general idea of this approach. First, massive libraries of DNA fragments are made using amplification. Thereafter, the fragments are washed with nucleotides that incorporate into the strands. Thereafter, the tags are scanned optically to determine the nucleotide they are attached to. An additional step of cleaving the tag away is also introduced which allows repeating the cycle several times; this process can be continued until the strand is completely read. This approach not only allows parallelization but also reduces the overall readout time significantly. Furthermore, with advances in 
(a)

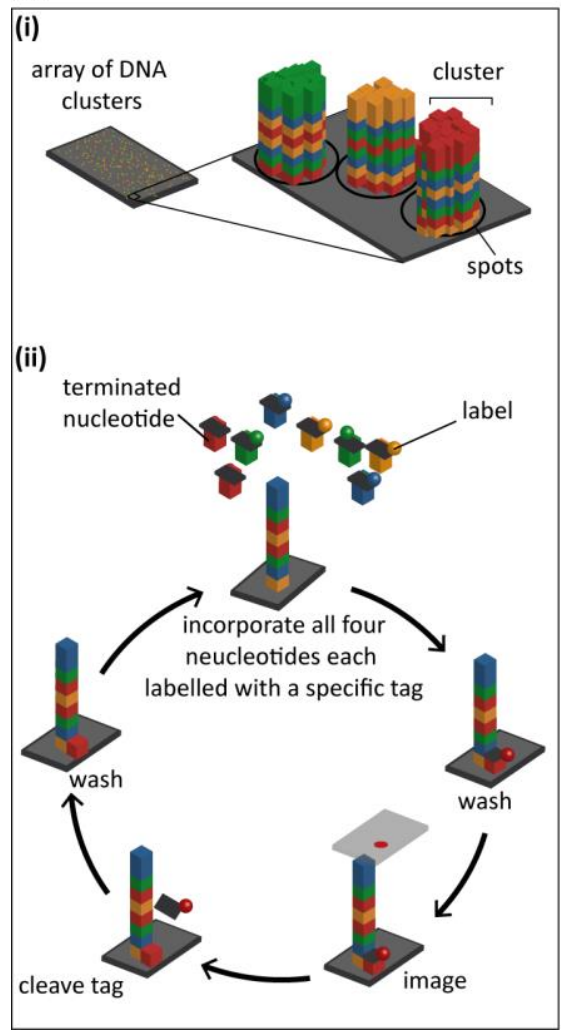

(b)

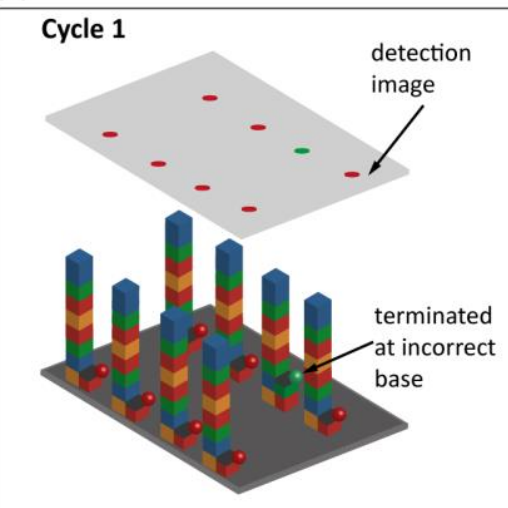

Cycle 8

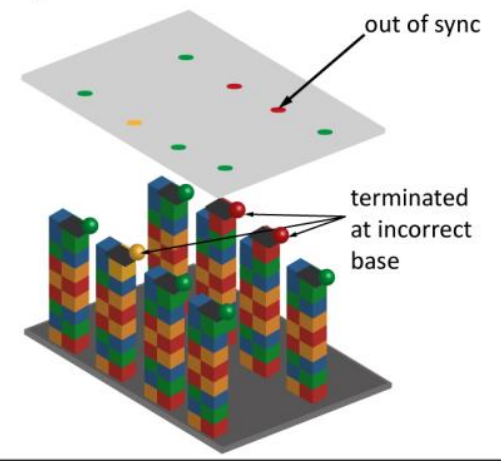

Figure 1.3. (a) Schematic diagram illustrating the wash and cycle method of a leading second generation sequencing technique (Illumina sequencing process). (i) The input DNA is split into several strands of varied lengths. These strands are amplified and attached on a substrate consisting of an array of spots. Each spots consists of a cluster of identical fragments. (ii) Second generation "wash-and-scan" method involves sequentially flooding the arrays with terminated nucleotides. Thereafter, the nucleotides get incorporated onto the strands one at a time as further growth is not possible owing to the terminators. The array is then washed to eliminate the background nucleotides. The nucleotides are labelled and their tags are color coded; identification of the individual nucleotide is possible via a unique optical signature. Finally, the terminating ends are cleaved away and washed again. This marks the end of a cycle. Scanning continues until the entire strand grows and each nucleotide incorporation is detected at every step. (b) Schematic showing an example of incorrect base incorporation within a cluster of fragments undergoing wash-and-scan cycle. Such an error may occur in one of more cycles at multiple strands. Error in any cycle gets propagated to following cycles and leads to error in base determination.

microfabrication processes, it was possible to condense the size of array-based 
design dramatically, thereby reducing the dosage of reagent from the scale of microliters to pico- or femtoliters. Thus, there was a significant reduction in costs of the reagents and the time of sequencing could be reduced from days to hours compared to FGS methods when using similar read lengths. SGS technology can now be used to sequence an entire human genome in a matter of days and at a cost of less than $\$ 10,000$. SGS methods are still commonly used and continue to be developed, having gained commercial success as well. ${ }^{3-4,6-7}$ They have outperformed FGS by orders of magnitude in throughput and reduced the cost of sequencing to between $4 \%$ and $0.1 \%$ of the original costs. ${ }^{3}$

Despite the progress in SGS techniques, accuracy is still a limitation. ${ }^{5,11-16}$ As discussed earlier, the input DNA is fragmented into several snippets and the sequence of these individual pieces is identified. The overall sequence can be reconstructed by assembling the individual pieces. However, errors are unavoidable during both these processes. For example, when a cluster of identical fragments are scanned simultaneously, ideally they must all produce the same signal. In practice, however, if for a particular strand at any given cycle, the base does not get terminated and leads to an additional incorporation of nucleotide, then the signal obtained from all fragments would be identical except one (as shown in Figure 1.3b). If the nucleotide incorporation occurs normally in the following cycles, then the position of the remaining nucleotides would skip a position in the readout (or be outof-sync) after the point of incorrect-base termination. However, this aberration may occur over many cycles and in multiple strands. Furthermore, the nature of the anomaly differs between strands (deletion, insertion, etc.). The error due to PCR increases with increasing lengths and thus SGS technologies usually apply to small read lengths (a few hundred bases per read length). Multiple fragments can be read simultaneously and their readouts can be combined for the reconstruction of the sequence. Although this reduces the error significantly, shorter read lengths lead to huge volumes of data. Complex and time consuming assembly algorithms and post processing are used to integrate data from individual fragments to derive a meaningful overall sequence. While it has been possible to minimize the errors, algorithms for reconstruction of error-free sequencing, de novo sequencing (in which the genome sequence of an organism is discovered for the first time), in particular, continue to be researched and refined. ${ }^{14-16}$ 


\subsection{Third-generation sequencing}

With the rapid pace of development it is no surprise that third generation sequencing (TGS) techniques are already on the horizon. Although there is no general consensus on the absolute best sequencing technique at this time. ${ }^{3,17-18}$ TGS aims at improving two main aspects of present-day technology ${ }^{3,18}$ : minimizing error and sequencing time. Both goals are achieved by obviating DNA amplification altogether and introducing single-molecule sequencing techniques with very long read lengths (thousands of bases per read length) in which readout occurs during nucleotide incorporation. A notable technique is that of Pacific Biosciences, ${ }^{12-13}$ a commercially available TGS sequencer at present. It introduced the single-molecule real-time sequencing (SMRT) approach that enables direct observation of single nucleotides as they incorporate to the DNA strand by the polymerase enzyme. It works on the principle of so-called zero-mode wave guides consisting of wells a few tens of zeptolitres in volume. At this exceedingly small scale, a single molecule can be illuminated while excluding the labelled nucleotides in the background, thereby eliminating the need for wash steps. The longest read length reported at present employing this approach is more than $10 \mathrm{kbp}$ (kilo base pairs). ${ }^{3,18-19}$

DNA sequencing with nanopores is another technology that has been researched and was first suggested even before the SGS techniques had surfaced. It was demonstrated that a single stranded DNA could be driven across a lipid bilayer through ion channels by electrophoresis. ${ }^{18}$ Current through the channel was observed and the passage of the strand blocked the ion flow thereby decreasing the current for a length of time that was proportional to the length of the strand. ${ }^{20}$ Oxford Nanopore Technology is the first company that offer nanopore sequencers; ${ }^{16,21}$ here a DNA strand is passed through a nanometer-scale aperture in a thin membrane. As the bases pass through the pore, the ionic flow decreases thereby reducing the current distinctly based on the nucleotides passing through. This was further expanded to create solid-state technology to recreate non-biological nanopores for the purpose of monitoring ion currents. ${ }^{22}$ Such methods provide the opportunity to fully exploit the high catalytic rates of DNA polymerases, ${ }^{3}$ unlike the SGS, where the strands are amplified into clusters and detected in a phased approach. Yet another method of direct imaging of DNA using local-probe imaging techniques such as scanning tunneling microscopy is being explored, ${ }^{23}$ but no practical implementation of sequencing using this approach exists at present. 
The long read lengths of TGS ease the process of reconstruction of the overall sequence relative to smaller fragments for several reasons. As the overall genome sequence consists of repetitive and non-repetitive sequences, it is a tedious problem to reconstruct the original sequence with short repetitive fragments. However, longer fragments anchor several repetitive sequences to their surrounding nonrepetitive sequences. If the reads are long enough, then the overall sequence can be reconstructed unambiguously by overlapping non-repetitive sequences. Thus, this generation of sequencing techniques is superior to the previous ensemble-based

(a)
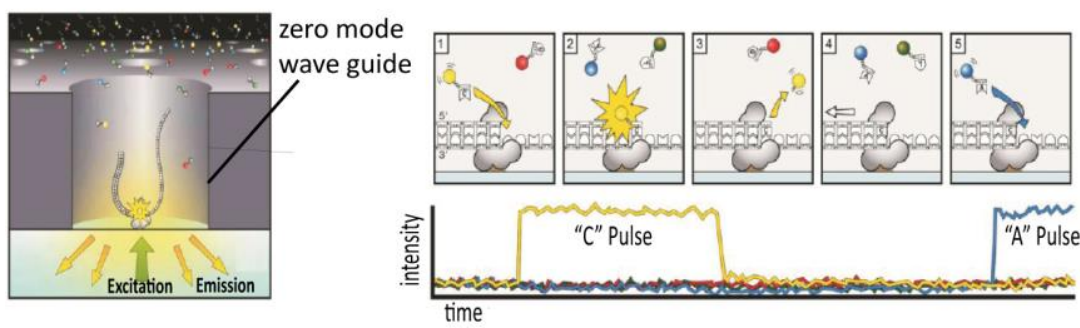

(b)

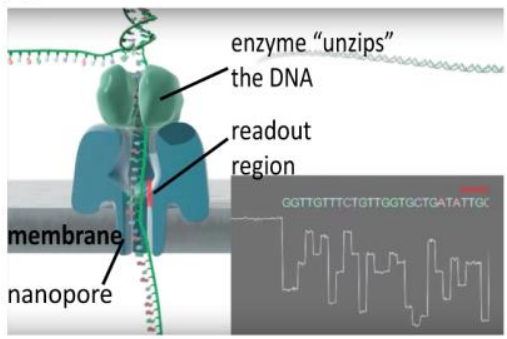

(c)

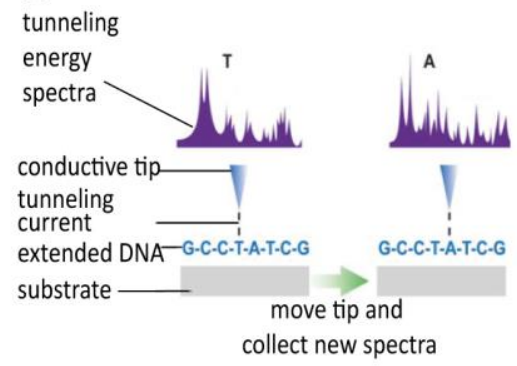

Figure 1.4. Schematic of the third generation DNA-sequencing techniques. (a) Pacific Biosciences method using zero mode wave guide wherein addition of fluorescent labelled nucleotides are detected. Reproduced from Eid et al. ${ }^{24}$ (b) Oxford Nanopores Technology using a nanopore inserted in a thin membrane (lipid bilayer) across which a voltage is applied. The ionic current through the nanopore is monitored. As the DNA strand passes through the nanopore, different bases restrict the flow of current in distinct manners and this allows a fingerprint of the sequence of the molecule to be extracted. Reproduced from Oxford Nanopore Technology. ${ }^{21}$ (c) A proposed DNA sequencing technique by direct inspection using scanning tunneling microscopy (STM). The DNA is first stretched and thereafter inspected at the atomic scale by STM. Reproduced from Schadt et al. ${ }^{3}$ 
technologies. This opens avenues for de novo sequencing as well. The reagents used are also much lower than the SGS methods and leads to a reduction of costs as well. However, TGS introduces a different nature of errors. As each base is monitored in real-time, raw read errors omissions (that is, missed bases) are more likely. Although the error profile is more uniform in TGS compared to the previous techniques, new analysis tools are being developed to derive high accuracies during real-time detection. ${ }^{3}$ While there is scope for development in detection methods and data analysis, the overwhelming advantages of TGS have stirred the research community to develop technologies that can ultimately be used in ultra-portable and low-cost full genome sequencing systems. Fast and low-cost full genome DNA sequencers have the potential to be a part of affordable healthcare, environment, food and livestock analysis.

\subsection{Single molecule real-time electronic sequencing}

In this thesis, we concentrate on the development of a single-molecule detection technique that has the potential to be used as a tool for third generation sequencing. This work was inspired by a program of the National Human Genome Research Institute (NHGRI), part of the National Institutes of Health (NIH, USA), which aims at building an all electrical sequencing platform.

\subsubsection{Electrochemical sensing}

This method relies on electrochemical reactions within nanogaps or nanofluidic channels for sensing. The nanogaps consist of two parallel electrodes separated by a few tens of nanometers. ${ }^{25-28}$ Their use for single-molecule detection has been demonstrated in the past. ${ }^{29}$ This method relies on charge amplification from electroactive molecules due to successive oxidation and reduction. DNA sequencing can in principle be achieved using specifically engineered tags that are electroactive in nature. Thus electrochemical identification of the tag can be representative of a specific nucleotide. Two schemes have been proposed to achieve this.

Scheme 1. It is proposed that the DNA strand being sequenced is loaded with a polymerase consisting of nucleotides that are labelled with four tags corresponding to the four base pairs. During the polymerase reaction, as the nucleotide is incorporated into the strand, a specific tag is released into the sensor. The tags become electroactive only after incorporation of the nucleotide. Figure 1.5a 
illustrates the detection scheme of such a platform that delivers an electrical (current) signal as the output.

(a) Scheme 1

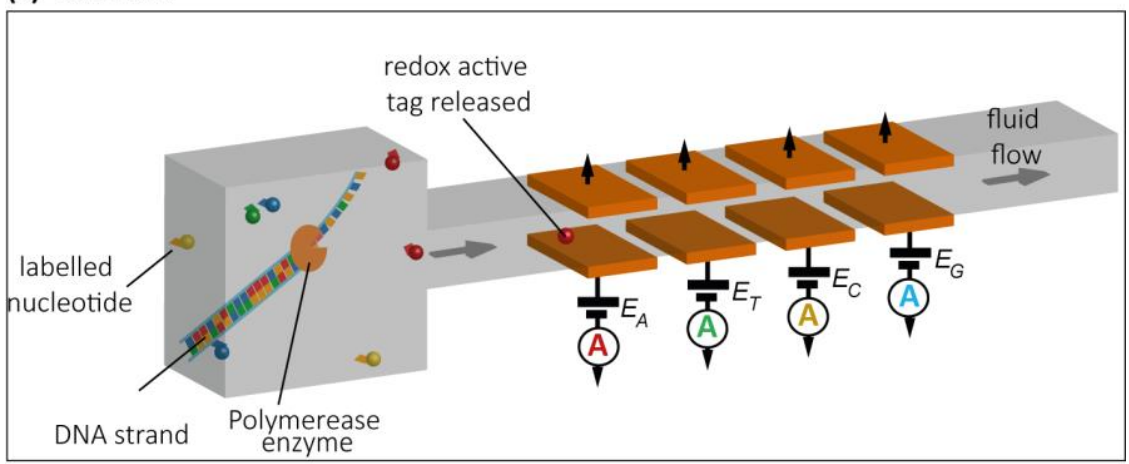

(b) Scheme 2

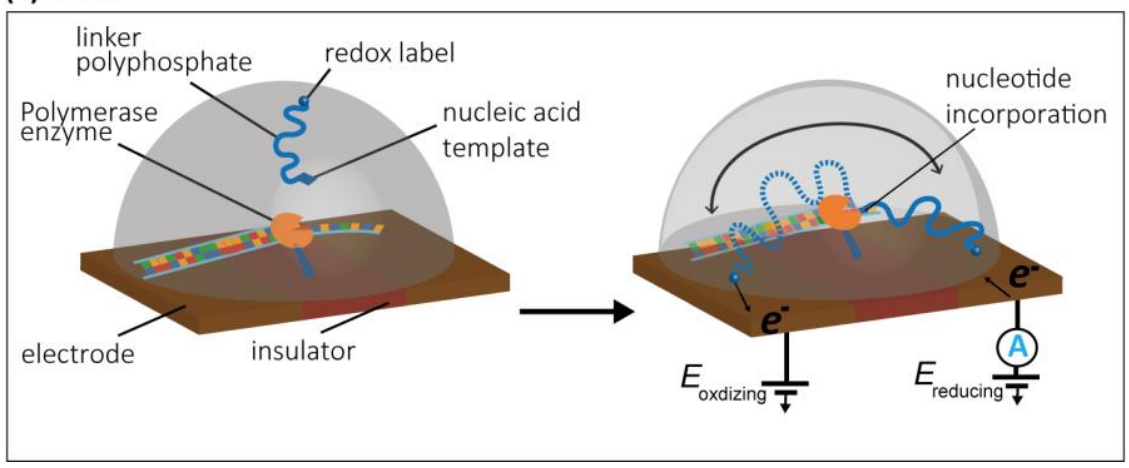

Figure 1.5. (a) Scheme 1: schematic diagram for a DNA sequencing platform based on electrochemical sensing of unique electroactive tags for the four nucleotides. When complementary labelled nucleotides are incorporated into the polymerase, the tags are released into the sensor. Detection is based on either amperometric or potentiometric methods to deliver a readout signal in terms of current or voltage, respectively. The nucleotides can be separated based on the potential at which the tag is activated. (b) Scheme 2: schematic diagram for a DNA sequencing platform based on electrochemical detection of electroactive tags attached to the four nucleotides. DNA polymerase is attached to an insulating surface. When a complementary nucleotide is incorporated into the polymerase, the redox tag oscillates between the two electrodes and provides an amplified signal. Upon base incorporation the phosphate is cleaved off and the process is repeated for the next nucleotide. ${ }^{30}$ 
Scheme 2. The DNA strand to be sequenced is attached to an insulating substrate within a planar sensor consisting of two closely spaced electrodes, as shown in Figure 1.5b. Artificial nucleotides incorporate a redox tag attached to a flexible polymer. Upon incorporation of a nucleotide to the DNA strand attached on the surface via a polymerase enzyme, the redox tag can oscillate multiple times between the two electrodes before the phosphate cleaves and diffuses away, yielding an electrical signal.

\subsubsection{Electronics integration}

Because DNA sequencing requires generating large quantities of data in a parallelized manner, it is natural to consider implementing the readout mechanism using integrated circuits. Complementary metal-oxide-semiconductor (CMOS) technology, which forms the basis for the vast majority of modern digital electronics, is well suited to applying desired voltages at electrodes and reading out the resulting currents or potentials. ${ }^{31}$ Signal processing, analog-to-digital conversion and data storage can all be incorporated into the system as well. This makes it possible to consider a highly multiplexed and parallelized sequencing platform. Care must however be taken to keep the fabrication processes for electronic and electrochemical devices compatible.

\subsection{Outline of the thesis}

The scope of this thesis far exceeds DNA sequencing as the techniques developed here are applicable to a wide range of situations. Electrochemical sensors have proven to be a powerful tool in analytical chemistry and have been a mainstay for decades owing to their remarkable detectability and experimental simplicity. ${ }^{32}$ Thus such sensors, either standalone or integrated with fluidics, have found a leading position among presently available commercial sensors in such fields as clinical, industrial and environmental analysis. ${ }^{33-35}$ While there are innumerable examples wherein such sensors can be particularly useful for commercialization, in this thesis we address several challenges specific to scaling such sensors down to micro/nanoscales.

One such aspect is detection at low limits of detection (LOD), especially at trace levels. For instance, in point-of-care (PoC) diagnostics, ${ }^{36-38}$ detection of trace levels 
of protein biomarkers in serum is crucial for early diagnosis of diseases such as cancer. This approach can further be expanded to offer personalized drugs that can be monitored also using biomarker-based monitoring to improve therapeutic outcome for patients. Pollution control is another such example, as there is a need for technologies that are capable of monitoring, recognizing and, ideally, removing small amount of contaminants present in air, water and soil. ${ }^{39}$ Detection at microscales is gaining much importance owing to their ability to perform very sensitive detection over conventional detection methods. ${ }^{40-43}$

In this thesis, we explore the individual ingredients for a fully integrated electrochemical biosensing platform with ultra-low LOD such as the one described in section 1.4 above. For this we tackle some of the practical challenges for realizing systems and propose and demonstrate alternative solutions.

Chapter 2 introduces electrochemistry in the context of micro/nanofluidic devices for miniaturized sensors. Core concepts are introduced and the non-idealities that one encounters upon scaling down (some of which are not noticeable in the macro domain) are highlighted.

Chapter 3 addresses the significance of a reference electrode in electrochemical measurements. As it remains a challenge to implement a reliable reference electrode in miniaturized systems, this chapter introduces an alternative technique: a reference-free system.

Chapter 4 discusses a potentiometric method, introduced as an alternative to amperometric method, for the electrochemical detection of redox molecules. The method is introduced on a proof-of-concept level and experimental results are presented.

Chapter 5 introduces a new generation of nanogap sensors that are integrated with microfluidic channels based on SU-8 and glass wafer bonding. The incorporation of flow is a prerequisite for the sequential detection of multiple analytes at the singlemolecule level (scheme 1 in Figure 1.5a), and the use of glass has the further benefit of minimizing contamination. In order to demonstrate the functioning of flowincorporated devices, dopamine measurements are described.

Chapter 6 demonstrates the detection of flexible, linear poly(ethylene glycol) polymers which are functionalized with redox-active moieties inside the nanogap devices. This represents the key building block of scheme 2 discussed above (Figure $1.5 \mathrm{~b})$. Depending on the length of the polymer, the end groups allow the transfer of 
electrons between the two electrodes which can be detected from the resulting cycling current.

\subsection{References}

1. Watson, J.D.; Crick, F.H.C., The Structure of DNA. Cold Spring Harb Sym 1953, 18, 123-131.

2. Air, G.M.; Sanger, F.; Barrell, B.G.; Brown, N.L.; Coulson, A.R.; Fiddes, J.C.; Hutchison, C.A.; Slocombe, P.M.; Smith, M., Nucleotide-Sequence of DNA of Bacteriophage-Phix174. P Aust Biochem Soc 1977, 10, 60-60.

3. Schadt, E.E.; Turner, S.; Kasarskis, A., A window into third-generation sequencing. Hum Mol Genet 2010, 19, R227-R240.

4. Lander, E.S., et al., Initial sequencing and analysis of the human genome. Nature 2001, 409, 860-921.

5. Hiatt, J.; Turner, E.; Patwardhan, R.; Lee, C.; Shendure, J., Next-Generation DNA Sequencing for De Novo Genome Assembly. J Invest Med 2009, 57, 99-100.

6. Mardis, E.R., Next-generation DNA sequencing methods. Annu Rev Genom Hum G 2008, 9, 387-402.

7. Shendure, J.; Ji, H.L., Next-generation DNA sequencing. Nat Biotechno/ 2008, 26, 1135-1145.

8. Brenner, S., et al., Gene expression analysis by massively parallel signature sequencing (MPSS) on microbead arrays. Nat Biotechnol 2000, 18, 630-634.

9. A.H. van Vliet, A.H.M., Next generation sequencing of microbial transcriptomes: challenges and opportunities. Fems Microbiol Lett 2010, 302, 1-7.

10. Ronaghi, M.; Karamohamed, S.; Pettersson, B.; Uhlen, M.; Nyren, P., Real-time DNA sequencing using detection of pyrophosphate release. Anal Biochem 1996, 242, 84-89.

11. Sakai, H., et al., The power of single molecule real-time sequencing technology in the de novo assembly of a eukaryotic genome. Sci Rep-Uk 2015, 5.

12. Korlach, J.; Levene, M.; Foquet, M.; Turner, S.W.; Craighead, H.G.; Webb, W.W., Single molecule DNA sequence profiling in zero-mode waveguides using gammaphosphate linked nucleotide analogs. Biophys J 2003, 84, 141a-141a.

13. Korlach, J.; Levene, M.; Turner, S.W.; Craighead, H.G.; Webb, W.W., Single molecule analysis of DNA polymerase activity using zero-mode waveguides. Biophys J 2002, 82, 507a-507a.

14. Bayley, H., Nanotechnology Holes with an edge. Nature 2010, 467, 164-165.

15. Liu, H.T., et al., Translocation of Single-Stranded DNA Through Single-Walled Carbon Nanotubes. Science 2010, 327, 64-67.

16. Clarke, J.; Wu, H.C.; Jayasinghe, L.; Patel, A.; Reid, S.; Bayley, H., Continuous base identification for single-molecule nanopore DNA sequencing. Nat Nanotechnol 2009, 4, 265-270. 
17. Niedringhaus, T.P.; Milanova, D.; Kerby, M.B.; Snyder, M.P.; Barron, A.E., Landscape of Next-Generation Sequencing Technologies. Anal Chem 2011, 83, 4327-4341.

18. Heather, J.M.; Chain, B., The sequence of sequencers: The history of sequencing DNA. Genomics 2016, 107, 1-8.

19. van Dijk, E.L.; Auger, H.; Jaszczyszyn, Y.; Thermes, C., Ten years of nextgeneration sequencing technology. Trends Genet 2014, 30, 418-426.

20. Kasianowicz, J.J.; Brandin, E.; Branton, D.; Deamer, D.W., Characterization of individual polynucleotide molecules using a membrane channel. P Natl Acad Sci USA 1996, 93, 13770-13773.

21. Oxford Nanopore Technology. https://nanoporetech.com/sciencetechnology/how-it-works (accessed 20th July).

22. Dekker, C., Solid-state nanopores. Nat Nanotechnol 2007, 2, 209-215.

23. Blow, N., DNA sequencing: generation next-next. Nat Methods 2008, 5, 267-274.

24. Eid, J., et al., Real-Time DNA Sequencing from Single Polymerase Molecules. Science 2009, 323, 133-138.

25. Lemay, S.G.; Kang, S.; Mathwig, K.; Singh, P.S., Single-Molecule Electrochemistry: Present Status and Outlook. Accounts Chem Res 2013, 46, 369-377.

26. Lemay, S.K.a.S.G., Nanoelectrochemical Methods. In Nanoelectrochemistry, CRC Press: 2015; pp 573-600.

27. Zevenbergen, M.A.G.; Wolfrum, B.L.; Goluch, E.D.; Singh, P.S.; Lemay, S.G., Fast Electron-Transfer Kinetics Probed in Nanofluidic Channels. J Am Chem Soc 2009, 131, 11471-11477.

28. Kang, S.; Mathwig, K.; Lemay, S.G., Response time of nanofluidic electrochemical sensors. Lab Chip 2012, 12, 1262-1267.

29. Kang, S.; Nieuwenhuis, A.; Mathwig, K.; Mampallil, D.; Lemay, S.G., Electrochemical Single-Molecule Detection in Aqueous Solution Using SelfAligned Nanogap Transducers. Acs Nano 2013, 7, 10931-10937.

30. Korlach, J.; Turner, S.; Sun, L., Real-time redox sequencing. Google Patents: 2013.

31. Singh, P.S., From Sensors to Systems: CMOS-Integrated Electrocheimcal Biosensors. IEEE Access 2015, 3, 249-259.

32. Wang, J., Electrochemical detection for capillary electrophoresis microchips: A review. Electroanal 2005, 17, 1133-1140.

33. Kraly, J.R.; Holcomb, R.E.; Guan, Q.; Henry, C.S., Review: Microfluidic applications in metabolomics and metabolic profiling. Anal Chim Acta 2009, 653, 23-35.

34. Fragoso, A., et al., Integrated microfluidic platform for the electrochemical detection of breast cancer markers in patient serum samples. Lab Chip 2011, 11, 625-631.

35. Lin, Y.H.; Matson, D.W.; Bennett, W.D.; Thrall, K.D.; Timchalk, C., Integrated microfluidics/electrochemical sensor system for field-monitoring of toxic metals. Microreaction Technology: Industrial Prospects 2000, 588-596. 
36. Wan, Y.; Su, Y.; Zhu, X.H.; Liu, G.; Fan, C.H., Development of electrochemical immunosensors towards point of care diagnostics. Biosens Bioelectron 2013, 47, 1-11.

37. Chan, C.P.Y.; Mak, W.C.; Cheung, K.Y.; Sin, K.K.; Yu, C.M.; Rainer, T.H.; Renneberg, R., Evidence-Based Point-of-Care Diagnostics: Current Status and Emerging Technologies. Annu Rev Anal Chem 2013, 6, 191-211.

38. Rusling, J.F.; Kumar, C.V.; Gutkind, J.S.; Patel, V., Measurement of biomarker proteins for point-of-care early detection and monitoring of cancer. Analyst 2010, 135, 2496-2511.

39. Hanrahan, G.; Patil, D.G.; Wang, J., Electrochemical sensors for environmental monitoring: design, development and applications. J Environ Monitor 2004, 6, 657-664.

40. Jimenez-Jorquera, C.; Orozco, J.; Baldi, A., ISFET Based Microsensors for Environmental Monitoring. Sensors-Basel 2010, 10, 61-83.

41. Namour, P.; Lepot, M.; Jaffrezic-Renault, N., Recent Trends in Monitoring of European Water Framework Directive Priority Substances Using Micro-Sensors: A 2007-2009 Review. Sensors-Basel 2010, 10, 7947-7978.

42. Rassaei, L.; Amiri, M.; Cirtiu, C.M.; Sillanpaa, M.; Marken, F.; Sillanpaa, M., Nanoparticles in electrochemical sensors for environmental monitoring. TracTrend Anal Chem 2011, 30, 1704-1715.

43. Krystofova, O.; Trnkova, L.; Adam, V.; Zehnalek, J.; Hubalek, J.; Babula, P.; Kizek, R., Electrochemical Microsensors for the Detection of Cadmium(II) and Lead(II) lons in Plants. Sensors-Basel 2010, 10, 5308-5328. 



\section{Unconventional electrochemistry in micro/nanofluidic systems}

Electrochemistry is ideally suited to serve as a detection mechanism in miniaturized analysis systems. A significant hurdle can, however, be the implementation of reliable micrometer-scale reference electrodes. In this chapter, we introduce the principal challenges and discuss the approaches that have been employed to build suitable references. We then discuss several alternative strategies aimed at eliminating the reference electrode altogether, in particular two-electrode electrochemical cells, bipolar electrodes and chronopotentiometry.

The contents of this chapter are published as Sahana Sarkar, Stanley C. S. Lai and Serge G. Lemay, Unconventional electrochemistry in micro-/nano fluidic systems, Micromachines, 2016, 7(5), 81. 


\subsection{Introduction}

One of the main challenges in creating micro- and nanodevices for chemical analysis is downscaling the measurement system that is ultimately used for readout. Several features of electrochemistry render it a desirable mechanism for transducing chemical information into electrical signals. ${ }^{1-15}$ The fabrication of electrodes suitable for electrochemistry is largely compatible with the methods employed for creating micro- and nanofluidic channels, it requires minimal additional (relatively low-cost) equipment, its sensitivity often increases with the downscaling of the electrode dimensions, it directly yields electrical signals without an intermediary transduction step (e.g., light), and it operates at relatively low power. Nonetheless, electrochemical methods can prove challenging to implement in micro- and nanosystems: While the concepts and instrumentation required for such measurements are well developed on the macroscopic scale, subtle, unobvious adjustments and compromises are often necessary upon downscaling. This complexity often goes unrecognized in the design of miniaturized systems, limiting accuracy and performance.

The aim of this review is to introduce the key concepts that influence electrochemical measurements in micro- and nanoscale measurement systems. Our target audience consists of scientists and engineers working on miniaturizing electrochemical measurement systems. We assume that the reader is already familiar with the methods used to fabricate micro-/nanofluidic devices and with basic electrochemical principles, ${ }^{16,17}$ and concentrate on elucidating some of the key factors that influence electrochemical measurements in miniature systems. We pay particular attention to how the electrostatic potentials of electrodes are established, determined, and controlled - or not, as is often the case. We first discuss reference electrodes, a key component of most macroscopic electrochemical measurement systems. This allows introducing the notation used in the reminder of the article as well as some important concepts that are sometimes misunderstood. We then discuss two classes of systems in which the conventional electrode biasing scheme is abandoned, namely, electrochemical cells without a reference electrode and bipolar electrodes. We end with a brief discussion of potentiometric measurements, in which the potential of an electrode is not controlled but is instead employed for detection. Unless stated otherwise, we assume that the test solution consists of water containing both redoxactive analyte molecules as well as a much higher concentration of inert salt ions, the so-called supporting electrolyte. This situation is typical for, e.g., biomedical samples. 
We concentrate on fluidic devices and exclude individual miniature electrodes used in conjunction with macroscopic measurement cells, conventional electrodes modified with nanomaterials, and electrochemical scanning probe techniques, which are reviewed extensively elsewhere. ${ }^{18-21}$

\subsection{Anatomy of an electrode}

Before discussing specific electrochemical systems, we introduce a few key concepts that will recur throughout this review. ${ }^{1}$ The interface between a solution (an ionic conductor) and an electrode (an electronic conductor, typically a metal, but also potentially a semiconductor or a macromolecule) can be represented by a capacitor $C$ and a (nonlinear) resistor $R$ in a parallel configuration, as shown in Figure 2.1. Here, $C$ represents the buildup of charge in the so-called electrical double layer (EDL) that develops at this interface. The EDL consists of electrons (or holes) in the electrode and compensating ions in the solution. These lead to an electric field-and thus an electrostatic potential difference-between the solution and the electrode. The EDL is highly local, for example, extending only on the order of $\sim 1 \mathrm{~nm}$ for water at physiological concentrations. The resistor $R$, on the other hand, represents the transfer of electrons between the electrode and the redox species in solution via electrochemical reactions.

(a)

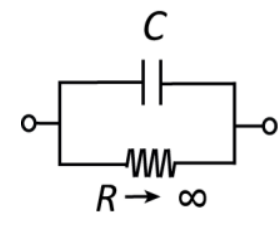

(b)

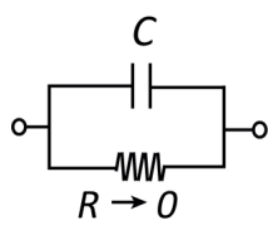

Figure 2.1. Equivalent circuits for (a) a polarizable and (b) a non-polarizable interface.

Electrodes can be qualitatively classified as polarizable or non-polarizable. In the case of a polarizable electrode, $R$ is very high and it is therefore possible to alter the potential difference across the interface without injecting significant current into the measurement cell. On the contrary, if $R$ is very low, changing the potential difference across the capacitor requires the application of very large currents, as charge is "leaked" through the interface. This short-circuit-like behavior is referred to as a nonpolarizable interface. In practice, no electrode is ever fully polarizable or nonpolarizable; whether an electrode represents a good approximation to either 
depends on the magnitude of the voltages and currents that occur in a particular measurement.

\subsection{Reference electrodes}

In macroscopic systems, electrochemical measurements are typically carried out in a three-electrode configuration, ${ }^{16}$ as shown schematically in Figure $2.2 \mathrm{a}$. The working (or indicator) electrode (WE) is the electrode where the analytical measurement takes place: An electrochemical reaction occurs if the potential difference between this electrode and the adjacent solution is such as to favor electron transfer, leading to a current. This electrode is coupled to an electrode of a known, defined potential, called the reference electrode (RE). The (conceptual) circuit diagram of this twoelectrode system is depicted in Figure 2.2b. Importantly, potentials applied to the WE are always with respect to the potential of the RE. Thus, an RE provides a reference point for the potential (similar to the role of ground in electronic circuits). However, it is important to note that the actual electrostatic potential difference between the $\mathrm{RE}$ and the solution may not be (and, in practice, rarely is) zero, and one therefore needs to specify the type of RE when stating the voltage of a WE (e.g., "1 V vs. $\mathrm{Ag} / \mathrm{AgCl}(3 \mathrm{M} \mathrm{KCl})$ " for a silver/silver chloride reference electrode immersed in a $3 \mathrm{M}$ potassium chloride solution). Similarly, an often overlooked nuance is that applying an external potential of $0 \mathrm{~V}$ with respect to the RE does not insure that no potential difference exists between the WE and the adjacent solution.

Any electrode system can serve as an RE as long as it approaches ideal nonpolarizability, meaning that its interfacial potential remains essentially fixed with the passage of currents. ${ }^{16,22}$ The amount of current that can pass depends on the specific RE system and design, but in general non-polarizability breaks down at "high" currents, ${ }^{22}$ and the reference potential will vary (for a commercial, macroscopic RE, this is typically in the order of $\mu \mathrm{A}^{\prime} \mathrm{s}$ ). Consequently, the WE potential is not controlled accurately at high currents, as a (undefined and variable) part of the applied potential between the WE and RE, E, is dropped at the RE-electrolyte interface. To circumvent this issue, one can introduce a third electrode, the counter (or auxiliary) electrode (CE). In this three-electrode setup, the current from the WE is routed through the CE, which acts as the electron source or sink for the reaction at the WE. The terminal controlling the RE has a high input impedance, rendering the current drawn through the RE negligible, and the RE interfacial potential thus remains constant. The technical implementation for potential control and current measurement in a three- 
electrode setup employs a potentiostat. Conceptually, this instrument monitors the potential difference between WE and RE, which is used as a feedback signal to control the current passing through the CE so that the actual potential difference matches the desired (applied) potential difference. A detailed description of the workings of a potentiostat can be found in many textbooks on electrochemistry and electrochemical instrumentation. ${ }^{16,23}$ As a final note, it should be borne in mind that a CE (and potentiostat by extension) is only required if the current in the system is large, and may be bypassed in miniaturized sensors if currents of the order of a few $\mu \mathrm{A}$ are measured that can be directly passed through a RE without significantly affecting its potential. In our experience, this condition is easily satisfied in most micro- and nanoscale systems. This results in compact simplified electronics, shown by the yellow box in Figure 2.2a, which essentially consists of a power source and an ammeter connected in series with the two electrodes.

(a)

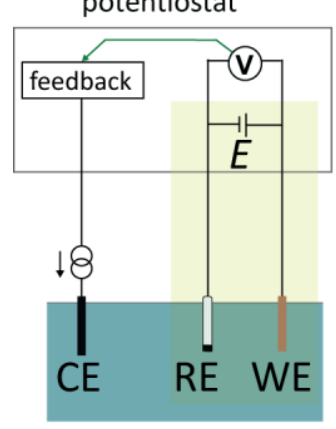

(b)

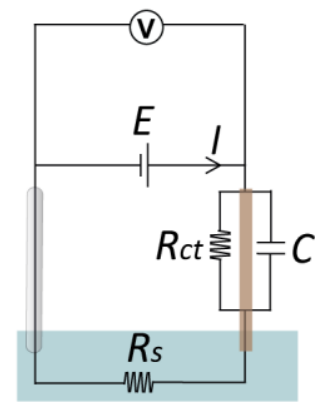

Figure 2.2. (a) Schematic of a conventional electrochemical cell for voltammetric measurement. The cell consists of three electrodes, termed the working (WE), reference (RE), and counter electrode (CE), immersed in the electrolyte solution. A potential, $E$, is applied to the WE with respect to the RE. If the current through the RE would be high enough to cause a potential shift, a CE is introduced to minimize the current through the RE. At low currents, it is instead possible to operate with a twoelectrode configuration and eliminate the CE altogether (highlighted in green), simplifying the detection circuitry. (b) Equivalent circuit diagram of a two-electrode setup. $R_{s}$ : solution resistance; $R_{c t}$ : charge-transfer resistance at the WE; $C$ : electrical double layer capacitance at the WE. This circuit treats the RE as ideally non-polarizable. 
Solution resistance. While in principle the RE only sets the electrostatic potential near its surface, the solutions employed in electrochemical measurements are ionic conductors. As a result, the potential of a solution when no electrical current is flowing through it is uniform throughout its entire bulk volume and is set by the RE. An important exception occurs at the boundaries of the liquid, where EDLs can develop as discussed above. This is particularly relevant near the surface of the WE, where a potential difference is required to drive electrochemical processes. However, if a net current, $I$, is flowing through the solution, an electric field can develop according to Ohm's law ( $E=I R_{s}$, where $R_{s}$ is the solution ionic resistance), and part of the applied voltage is dropped in the solution between the RE and WE. These ohmic voltage drops can be minimalized either by reducing the current (e.g., by decreasing the analyte concentration or reducing the size of the electrode) or by minimizing the electrolyte resistance between the RE and WE (e.g., by increasing the conductivity of the electrolyte solution or placing the RE close to the WE to decrease the length of the resistive path). In most electroanalytical measurements, the analyte concentration is much lower than the electrolytic (salt) concentration; therefore, these ohmic voltage drops may reasonably be neglected. However, if an electrolytic solution of low conductivity (usually due to low ionic strength) is used, $I R_{s}$ may be significant and needs to be taken into account when considering the WE potential $\left(E_{W E}=E-I R_{S}\right)$. This can be particularly significant in fluidic devices where confinement of the liquid easily leads to higher values of $R_{s}$ than is typical in macroscopic experiments.

Requirements. At this point, it is worth discussing the technical requirements of a reference electrode. A RE should have a potential which is stable over time ${ }^{22}$ and which is not significantly altered by small perturbations to the system - in particular, the passage of a small current. Some of the main considerations while designing a RE are discussed in depth by Shinwari et al. ${ }^{22}$ Commercial REs typically employ a macroscopic piece of metal (providing an "infinite" reservoir of redox species) coated with a sparingly soluble metal salt (such that the interfacial concentration is determined by the solubility product of the salt), immersed in a contained reference solution, and the entire system is connected to the test solution by a salt bridge (to prevent composition changes of the reference solution while minimizing the liquid junction potential). ${ }^{16,24}$ While such electrode systems are straightforward to realize on the macroscale, implementing REs in miniaturized systems requires careful considerations in the downscaling of all these components. ${ }^{22,25}$ 
Miniaturized REs. Several analogues to conventional REs have been demonstrated using microfabrication, and several techniques are available for their manufacture such as thin film deposition, ${ }^{26-30}$ electroplating, ${ }^{31,32}$ or screen printing ${ }^{33,34}$ of the metal followed by ion exchange reactions or electrochemical coating. The interface to the test solution and reference solution chamber is typically implemented using gels or nanoporous membranes/glass. For example, an $\mathrm{Ag} / \mathrm{AgCl}$ electrode was replicated by a thin-film deposition of $\mathrm{Ag}$ supported over $\mathrm{Pt}$, after which $\mathrm{AgCl}$ was formed by oxidizing it in a solution containing chloride ions. ${ }^{31}$ In another example, miniaturization of the liquid junction $\mathrm{Ag} / \mathrm{AgCl}$ was demonstrated by covering a deposited thin film of silver with a layer of polyamide. This layer had a slit at the center where $\mathrm{AgCl}$ was grown; the liquid junction was formed with photo-curable hydrophilic polymer. ${ }^{35}$

However, the stability of such miniaturized references electrodes is often limited, and typical problems include limited lifetimes, poor reproducibility, and drifting electrode potentials 22,36 . A common cause is the rapid consumption of the electrode material due to its small size. In general, electrode consumption can be divided into an electrochemical (Equation 2.1) and a chemical (Equation 2.2) pathway.

$$
\begin{gathered}
\mathrm{AgCl}(\mathrm{s})+\mathrm{e}^{-} \rightleftharpoons \mathrm{Ag}(\mathrm{s})+\mathrm{Cl}^{-}(\mathrm{aq}) \text { (electrochemical) } . \\
\left.\mathrm{AgCl}(\mathrm{s})+n \mathrm{Cl}^{-}(\mathrm{aq}) \rightleftharpoons \mathrm{AgCl}_{(n+1)}\right)^{n-}(\mathrm{aq}), \text { where } 0<n<3 \text { (chemical) } .
\end{gathered}
$$

In the electrochemical pathway, the passage of a small current through a miniaturized RE can already be sufficient to induce complete consumption of the electrode material within experimental time scales. For example, a microscopic $\mathrm{Ag} / \mathrm{AgCl} \mathrm{RE}$ of an area of $100 \mathrm{\mu m}^{2}$ (AgCl thickness $100 \mathrm{~nm}$ ) exposed to a current of only $10 \mathrm{pA}$ would be completely consumed within approximately one hour. The chemical pathway relates to the non-zero solubility of the metal salt, where the dissolved and solid species are only in chemical equilibrium as long as the solution is saturated with the metal salt. If the RE is exposed to a non-saturated solution, or the solution is continuously replenished (such as in flow systems), dissolution of the metal salt will occur. This issue is further exacerbated in the case of $\mathrm{Ag} / \mathrm{AgCl}$ electrodes, where there is a non-negligible formation of aqueous $\mathrm{AgCl}_{(n+1)}{ }^{n-}$ ion complexes in chloride-containing solutions. ${ }^{37,38}$ At physiological electrolyte concentrations, this leads to an equilibrium concentration of dissolved $\mathrm{AgCl}$ in the $\mu \mathrm{M}$ range, sufficient to completely dissolve a $100 \mu \mathrm{m}^{2} \times 100 \mathrm{~nm} \mathrm{AgCl}$ layer in $\sim 0.1 \mu \mathrm{L}$ of electrolyte solution. 
Another common cause for the limited stability of miniaturized REs is the possible contamination of the reference solution via non-ideal ("leaky") bridging membranes. This issue can be alleviated by eliminating the salt bridge and reference solutions. Such systems are commonly termed quasi- or pseudo-RE. While the terms are often used interchangeably, there is a subtle but important difference between the two. A quasi-RE simply omits the reference solution and immerses the electrode directly into the test solution. ${ }^{28,29,39-45}$ A clearly defined redox couple, however, sets the electrode potential, and any fluctuations result from changes in the activity coefficients of this couple. For example, a common $\mathrm{Ag} / \mathrm{AgCl}$ quasi-RE consists of a silver electrode coated with silver chloride salt and in contact with the chloridecontaining test solution; here, the $\mathrm{Ag} / \mathrm{Ag}^{+}$couple sets the solution potential. ${ }^{30}$ On the other hand, a pseudo-RE refers to a large surface area electrode (such as a platinum or silver wire) directly exposed to the solution. ${ }^{42,43,45}$ In this case, which redox couple sets the reference potential is undefined, and the reference potential remains reasonably constant by virtue of the large surface area, with even low reactivity being sufficient to take up small currents without significant polarization of the electrode. In both cases, the RE can be calibrated by measuring its potentials relative to a conventional RE. Thus, while miniaturizing REs still present challenges, rational design can provide a microscopic RE which is sufficiently stable given the requirements for a specific measurement.

Finally, it is worthwhile to consider the placement of electrodes in microfabricated systems. In a macroscopic system, the CE is placed far from the WE and RE, such that the substances produced at the CE do not reach the WE surface to interfere with the measurements there. However, in microscopic systems, this might not be possible due to space requirements, and such interference needs to be taken into account in order to avoid undesirable shifts in the reference potential.

\subsection{Systems without a reference electrode}

Considering the difficulties inherent in implementing miniaturized high-quality reference electrodes, it is natural that considerable effort has been devoted to creating analytical systems in which the role of the reference is minimized or omitted altogether. Doing so comes at a price since in such cases the interfacial potentials that drive electron-transfer reactions at the system's electrodes is no longer explicitly controlled. As a result, no universally applicable alternative to the conventional combination of potentiostat and reference electrode has evolved. Nonetheless, 
reliable alternatives can be implemented in some particular geometries and/or when sufficient information about the solution to be analyzed is available.

The basic configuration for a reference-free, two-electrode system is sketched in Figure 2.3. While this represents the simplest case of a system without an RE, the discussion of the solution potential in the following is general, and can be extended to incorporate additional electrode elements. The most important feature of the system of Figure 2.3 is that the interfacial potential differences at the two electrodes is not controlled separately since only the total potential difference between the two electrodes is accessible experimentally. The potential of the bulk electrolyte phase, $E_{s}$, is thus instead free to float to different values. This is in stark contrast with the case where one of the electrodes is an RE; in that case, there is no change in the potential difference at the RE interface, and the potential of the electrolyte is pinned to the RE potential.
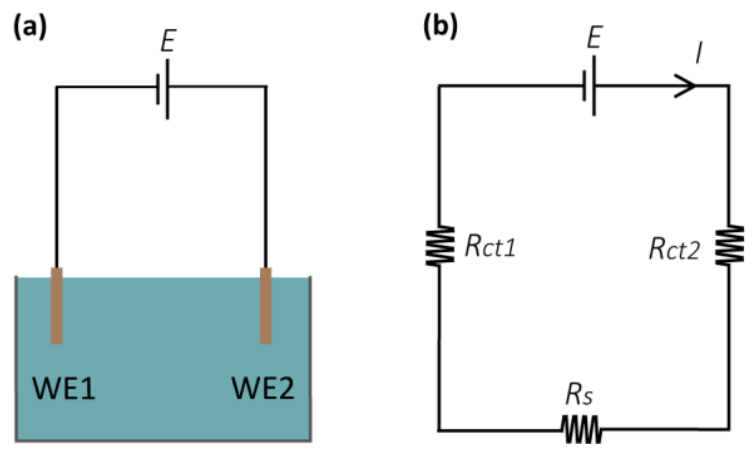

Figure 2.3. (a) Reference-less two-electrode system where $E$ is the applied potential between the two WEs. (b) Corresponding equivalent-circuit diagram. $R_{s}$ : solution resistance; $R_{c t 1,2}$ (charge transfer) resistance at the $W E_{1,2}$.

What sets the potential of the solution in the experiment of Figure 2.3? The passage of a current at one of the electrodes causes charge to be injected in this solution. As discussed above in the context of reference electrodes, this charge accumulates at the boundaries of the bulk phase. For example, an oxidation reaction taking place at an electrode causes the withdrawal of electrons from the solution and the accumulation of positive charge at its boundaries, in turn causing the electrostatic potential of the solution to become more positive. This acts as a negative feedback mechanism, as the shift in solution potential acts to inhibit the electrochemical process that caused it (in our example, the oxidation current decreases by making the solution more positive with respect to the electrode). The solution eventually 
settles to a stationary steady state at a potential such that no net charge injection takes place, that is, the total current being injected into the solution vanishes:

$$
\sum_{j} I_{j}=0, \text { where } I_{j}=I_{j}\left(V_{j}-E_{\mathrm{s}}\right)
$$

Here, $l_{j}$ is the current through the $j$ th electrode, which is a function of its interfacial potential difference $\left(V_{j}-E_{s}\right), V_{j}$ is the potential applied to the electrode, and $E_{s}$ is the solution potential (neglecting ohmic drops for ease of notation) with respect to a common reference point in the circuit such as signal ground. In principle, if the relations between current and interfacial potential at each of the electrodes are known (because, e.g., they can be derived from fundamental electrochemical kinetic theory or they have been experimentally determined), then it is possible to solve for the unique value of $E_{s}$ that satisfies Equation 2.3 and to deduce the current through each of the electrodes. This procedure essentially amounts to solving the equivalent circuit shown in Figure 2.3b, where the electrochemical reactions are represented by (highly nonlinear) resistors $R_{c t 1}$ and $R_{c t 2}$, and Equation 2.3 is the direct application of Kirchhoff's current law.

For the two-electrode system of Figure 2.3a, Equation 2.3 reduces to the statement that the solution potential will shift in such a way that the reduction current at the more negative of the two electrodes is equal in magnitude to the oxidation current at the more positive electrode. This scenario was discussed in detail by Xiong and White, ${ }^{46}$ where it was, for example, shown explicitly that increasing the area of one of the electrodes causes the solution potential to shift closer to that electrode's open-circuit potential because that electrode's effective resistance becomes smaller.

A further consequence of Equation 2.3 is that parasitic pathways for a current-such as may result from a minor leak-can sometimes have a significant influence in a microsystem without a reference electrode. In conventional electrochemical cells, such a parasitic current can be accommodated by the counter electrode (or the reference for low-current systems) without influencing the signal measured at the working electrode. For a floating solution potential, however, even relatively small uncompensated currents can lead to drift. This was illustrated by Sarkar et al., ${ }^{47}$ who showed how the (large) redox-cycling current between two electrodes separated by $65 \mathrm{~nm}$ can be controlled by the (much smaller) current to an additional electrode located outside the nanofluidic device. ${ }^{47}$ 


\subsection{Bipolar electrodes}

A bipolar electrode (BPE) is a floating conductor which facilitates opposing electrochemical reactions (oxidizing and reducing) on spatially separated regions of its surface. Two example systems are shown in Figure 2.4. Figure 2.4a represents the (conceptually) simplest case. Here, two electrolyte solutions are physically separated by a BPE, such that the only current path between them is through the BPE. Since it is a good conductor, the entire BPE is essentially at the same potential, while the relative potential of the two electrolyte solutions can be changed independently. Consequently, the local interfacial potential difference of the BPE with the adjacent solution is different at the two ends. If suitable species are present in the two reservoirs, reduction and oxidation processes may occur at the two ends of a BPE, thereby coupling two, otherwise isolated, electrochemical systems.

Alternatively, a BPE can be located in a single reservoir (Figure 2.4 b). Two additional electrodes are then placed at the ends of the reservoir, and applying a large current between them induces an electric field in the electrolyte due to its finite conductivity (ohmic drop). As shown in Figure 2.4b, this spatially heterogeneous solution potential leads to a gradient of electrostatic potential differences along the length of the BPE (that is, between the electrode and the solution). If a sufficiently large potential difference between the two ends of the bipolar electrode is induced, it becomes possible to drive an oxidation reaction at one end and a reduction at the opposite end of the same electrode, similarly to the case of Figure 2.4a.

(a)

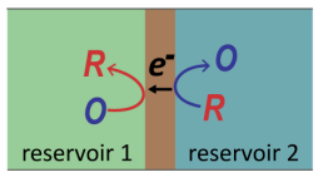

(b)

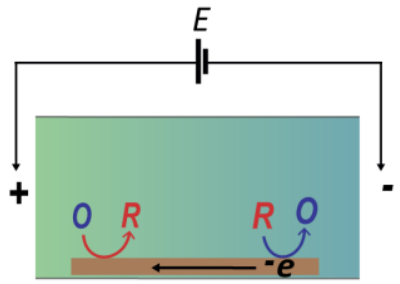

(c)

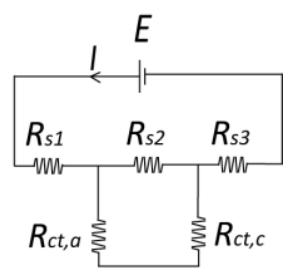

Figure 2.4. (a) Schematic diagram of a bipolar electrode (brown) in contact with two separate reservoirs. (b) Alternative concept of a bipolar electrode in which a uniform electric field is applied along a channel filled with electrolytic solution. A band electrode exposed to this solution exhibits bipolarity at its opposing ends (cathodic at left and anodic on right). (c) Equivalent circuit for panel (b). $E$ is the potential applied across the solution, $R_{s}$ is the resistance of the solution, and $R_{c t}$ is the charge transfer resistance across the anodic/cathodic ends of the bipolar electrode (BPE). 
From a purely conceptual point of view, the scenarios shown in Figures 2.3 a and 2.4 $b$ are very closely related. In each case, one element of the electrochemical circuitthe solution in Figure $2.3 \mathrm{a}$ and the BPEs in Figure 2.4-is free to adjust its electrostatic potential in response to redox reactions taking place at spatially separated regions. It is therefore unsurprising that the same basic principles apply for determining the potential to which the BPE drifts in response to electrochemistry. In fact, Equation 2.3 carries over directly to this case, where now $E_{s}$ represents the potential of the bipolar electrode, and $j$ is an index that runs over the different regions of this electrode (for the case of a continuous gradient as in Figure 2.4b, the sum becomes an integral over the electrode surface, but the underlying principle remains unchanged).

The defining feature of BPEs is that they are floating electrodes, yet can be induced to facilitate electrochemical reactions of choice at their interface. This is particularly attractive for miniaturized systems, as abolishing the need for contacts to solution (i.e. reference electrodes) simplifies fabrication and instrumentation. Furthermore, it enables an arbitrarily large number of BPEs (such as arrays of BPEs imbedded in insulating matrices ${ }^{48}$ ) to be driven simultaneously. The use of BPEs in the micro/nanoscopic domain was pioneered by Bradley et al. ${ }^{49}$ who demonstrated the use of bipolar electrochemistry to create electrical contacts in microcircuits by employing copper electrodeposition as the cathodic reaction. This work was followed by a dramatic increase in the investigation of bipolar electrochemistry-in particular, by the groups of Kuhn ${ }^{50-55}$ and Crooks. ${ }^{56-59}$ A recent review by Sequeira et al. ${ }^{60}$ discusses bipolar electrochemistry and their many varied applications that several contemporary groups are presently exploring. As a particularly striking example, Mallouk, Sen, and colleagues ${ }^{61-63}$ demonstrated a locomotion mechanism for bipolar microswimmers based on electrochemical reactions taking place at both ends of the swimmer. Another intriguing variant is to use the bipolar electrode to couple the reaction of a target analyte to a second, separate reaction that produces an optically active species. Using the latter's fluorescent properties allowed for the demonstration of the highly sensitive, fluorescence-mediated detection of species that are not themselves optically active. ${ }^{48,64}$

Implicit bipolar behavior. Apart from devices that explicitly exploit bipolar electrochemistry as their mode of operation, this effect has an important consequence for the design and validation of electrochemical detection devices. Any conductor in contact with solution has the potential to act as a bipolar electrode if its 
potential is not controlled. This is a very different situation from conventional electronic devices, where leaving a particular component unconnected typically means that it can be safely ignored, at best, or a source of stray capacitance, at worse. A well-documented example of a system where bipolar electrochemistry is implicitly utilized is scanning electrochemical microscopy (SECM in the positive feedback mode), where a conducting sample can be left unbiased but then acts as a bipolar electrode. ${ }^{65,66}$ Similarly, floating electrodes imbedded in nanochannels were shown to act as "short circuits" to a reference located outside the nanofluidic device. ${ }^{47,67}$ Last but not least, it is important to keep in mind that all solvents-especially waterare liable to electrochemical breakdown; if a sufficient potential gradient is applied, any floating metal features in a device can become implicated in reactions involving water, protons, hydroxide, or dissolved oxygen, leading to unintended currents flowing through the system. ${ }^{68,69}$

\subsection{Potentiometry}

The main theme of this review has been the control of potentials in electrochemical systems. For completeness, we discuss here very briefly potentiometry, the branch of analytical chemistry concerned with the measurement of potentials as detection mechanism. It is difficult to understate the importance of potentiometry as it forms the basis for many widely used technologies, starting with $\mathrm{pH}$-sensitive electrodes and extending to a wide family of other ion-selective electrodes. ${ }^{70-73}$ In its most common form, potentiometry is an equilibrium technique, with the potential of a working electrode being measured with respect to a reference. This makes it particularly sensitive to the choice of RE, which becomes challenging to implement in miniaturized systems given all the complications discussed above. Commonly used for concentration determination, lower detection limits of such techniques can be achieved with downscaling, and extensive work has been carried out in the development of so-called nanopotentiometry. Much of this work has focused on nanostructured thin films interfaced to macroscopic electrodes. ${ }^{70,72}$ To what extent these approaches and materials can be adapted in the context of, e.g., lithographically fabricated micro- and nanodevices largely remains an interesting question for future work. Thus, while this is an area where we expect major developments will likely happen in the near future, we do not attempt to discuss specific works at this time. 
One variant that may lend itself more readily to integrated miniature systems is socalled chronopotentiometry ${ }^{12}$, in which the potential of an electrode is monitored as a function of time using high-impedance readout circuitry. Before equilibrium is established, electrochemical reactions occurring at an electrode cause its potential to shift over time. The rate of change of the potential is proportional to the electrochemical current and inversely proportional to the electrode capacitance; hence, a concentration can in principle be extracted from the time-dependent data. To explicitly illustrate this principle, we show in Figure 2.5 measurements of the potential of a floating electrode over time as it accumulates charge due to redox cycling in a nanofluidic device ${ }^{47}$ (consisting of two electrodes, one of which is floating). The evolution of the potential over time reflects the rate of electrochemical charge transfer, which itself depends on the composition of the solution.

(a)

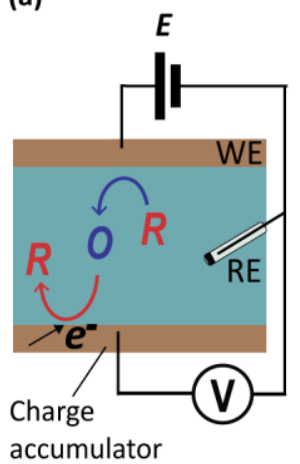

(b)

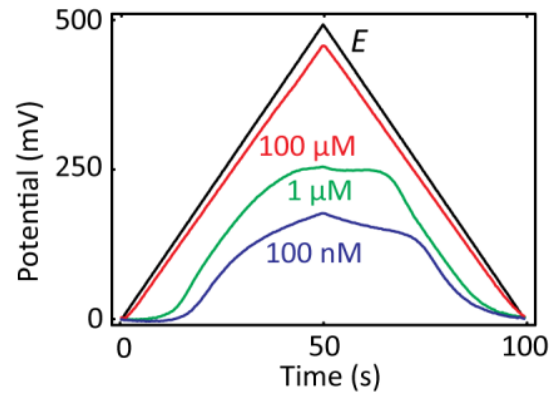

Figure 2.5. (a) Schematic diagram of a two-electrode nanogap system in contact with a solution containing reversible redox species. The bottom electrode accumulates charge over time, and the resulting potential shift is used as readout signal. (b) Chronopotentiometric signal versus concentration of redox species $(100 \mu \mathrm{M}, 10 \mu \mathrm{M}$, and 1 $\mathrm{nM} \mathrm{Fc}(\mathrm{MeOH})_{2}$ in $\left.0.1 \mathrm{M} \mathrm{KCl}\right)$ in response to a triangular potential wave applied to the top electrode (black line).

Furthermore, since small electrodes normally have lower capacitances, the potentiometric signal is more sensitive in this case, making the method a logical candidate for miniaturization. Based on the additional consideration that the readout of potentials is relatively straightforward to implement in conventional CMOS electronics ${ }^{74}$, Zhu et al. $^{75-77}$ suggested that chronopotentiometry is particularly well suited for systems in which fluidics and electronics are implemented on a single, 
highly integrated chip. Whether this type of electrochemical analysis can offer a competitive alternative to existing methods is presently an open question.

\subsection{Summary and outlook}

The emergence of point-of-care diagnostic systems has led to the rapprochement of micro-/nanofluidics and electrochemical sensing methods, and this trend can be expected to strengthen in the coming years. Although electrochemistry is an exhaustive subject and a vast amount of information is available in the literature, it is not always straightforward for new researchers in the area of microsystems to identify the concepts and approaches that are most relevant for building practical miniaturized devices. This is particularly true because some standard ingredients of electrochemical analysis - especially the use of optimized reference electrodes-are surprisingly challenging to scale down. While universally applicable solutions have yet to emerge, many common pitfalls can be avoided by informed experimental design. We have thus attempted to provide an introduction to the methods of micro-/nanoelectrochemistry and, in particular, to make the reader aware of nonidealities which are not necessarily obvious when extrapolating from the macro domain. We hope that linking some of the concepts addressed in this paper will be beneficial to the fluidic sensor community and will help to stimulate further exploration of the rich field of miniature sensing technologies.

\section{$2.8 \quad$ References}

1. Oja, S.M.; Fan, Y.S.; Armstrong, C.M.; Defnet, P.; Zhang, B. Nanoscale electrochemistry revisited. Anal. Chem. 2016, 88, 414-430.

2. Oja, S.M.; Wood, M.; Zhang, B. Nanoscale electrochemistry. Anal. Chem. 2013, 85, 473-486.

3. Watkins, J.J.; Zhang, B.; White, H.S. Electrochemistry at nanometer-scaled electrodes. J. Chem. Educ. 2005, 82, 712-719.

4. Rackus, D.G.; Shamsi, M.H.; Wheeler, A.R. Electrochemistry, biosensors and microfluidics: A convergence of fields. Chem. Soc. Rev. 2015, 44, 5320-5340.

5. Rassaei, L.; Singh, P.S.; Lemay, S.G. Lithography-based nanoelectrochemistry. Anal. Chem. 2011, 83, 3974-3980.

6. Meier, J.; Schiotz, J.; Liu, P.; Norskov, J.K.; Stimming, U. Nano-scale effects in electrochemistry. Chem. Phys. Lett. 2004, 390, 440-444.

7. Arrigan, D.W.M. Nanoelectrodes, nanoelectrode arrays and their applications. Analyst 2004, 129, 1157-1165. 
8. Kang, S.; Lemay, S.G. Nanoelectrochemical methods. In Nanoelectrochemistry; Mirkin, M.V., Amemiya, S., Eds.; CRC Press: Boca Ratan, USA, 2015; pp. 573-600.

9. Micheal, MV.; Amemiya, A. Nanoelectrochemistry; CRC Press: Boca Ratan, USA, 2015.

10. Lee, T.M.H. Over-the-counter biosensors: Past, present, and future. Sensors 2008, 8, 5535-5559.

11. Bakker, E.; Qin, Y. Electrochemical sensors. Anal. Chem. 2006, 78, 3965-3983.

12. Grieshaber, D.; MacKenzie, R.; Voros, J.; Reimhult, E. Electrochemical biosensors-Sensor principles and architectures. Sensors 2008, 8, 1400-1458.

13. Ino, K. Microchemistry- and MEMS-based integrated electrochemical devices for bioassay applications. Electrochemistry 2015, 83, 688-694.

14. Wei, D.; Bailey, M.J.A.; Andrew, P.; Ryhanen, T. Electrochemical biosensors at the nanoscale. Lab Chip 2009, 9, 2123-2131.

15. Gencoglu, A.; Minerick, A.R. Electrochemical detection techniques in micro- and nanofluidic devices. Microfluid. Nanofluid. 2014, 17, 781-807.

16. Bard, A.J.; Faulkner, L.R. Electrochemical Methods: Fundamentals and Applications; Wiley: New York, USA, 2000.

17. Brett, C.M.A.; Brett, A.M.O. Electrochemistry: Principles, Methods, and Applications; Oxford University Press: Oxford, UK, 1993.

18. Moore, A.M.; Weiss, P.S. Functional and spectroscopic measurements with scanning tunneling microscopy. Annu. Rev. Anal. Chem. 2008, 1, 857-882.

19. Mirkin, M.V.; Nogala, W.; Velmurugan, J.; Wang, Y.X. Scanning electrochemical microscopy in the 21st century. Update 1: Five years after. Phys. Chem. Chem. Phys. 2011, 13, 21196-21212.

20. Sun, P.; Laforge, F.O.; Mirkin, M.V. Scanning electrochemical microscopy in the 21st century. Phys. Chem. Chem. Phys. 2007, 9, 802-823.

21. Macpherson, J.V.; Unwin, P.R. Noncontact electrochemical imaging with combined scanning electrochemical atomic force microscopy. Anal. Chem. 2001, 73, 550-557.

22. Shinwari, M.W.; Zhitomirsky, D.; Deen, I.A.; Selvaganapathy, P.R.; Deen, M.J.; Landheer, D. Microfabricated reference electrodes and their biosensing applications. Sensors 2010, 10, 1679-1715.

23. Hickling, A. Studies in electrode polarisation part IV-The automatic control of the potential of a working electrode. Trans. Faraday Soc. 1942, 38, 27-33.

24. Brezinski, D.P. Kinetic, static and stirring errors of liquid junction reference electrodes, Analyst, 1983, 108, 425-442.

25. Mousavi, M.P.S.; Buhlmann, P. Reference electrodes with salt bridges contained in nanoporous glass: An underappreciated source of error. Anal. Chem. 2013, 85, 8895-8901.

26. Kim, T.Y.; Hong, S.A.; Yang, S. A solid-state thin-film Ag/AgCl reference electrode coated with graphene oxide and its use in a pH sensor. Sensors 2015, 15, 64696482. 
27. Webster, T.A.; Goluch, E.D. Electrochemical detection of pyocyanin in nanochannels with integrated palladium hydride reference electrodes. Lab Chip 2012, 12, 5195-5201.

28. Matsumoto, T.; Ohashi, A.; Ito, N. Development of a micro-planar ag/agcl quasireference electrode with long-term stability for an amperometric glucose sensor. Anal. Chim. Acta 2002, 462, 253-259.

29. Uludag, Y.; Olcer, Z.; Sagiroglu, M.S. Design and characterisation of a thin-film electrode array with shared reference/counter electrodes for electrochemical detection. Biosens. Bioelectron. 2014, 57, 85-90.

30. Rivas, I.; Puente, D.; Ayerdi, I.; Castano, E. Ag/Agl quasi-reference microelectrodes. In Proceedings of the 2005 Spanish Conference on Electron Devices, Tarragona, Spain, 2-4 Febuary 2005; pp. 465-468.

31. Zhou, J.H.; Ren, K.N.; Zheng, Y.Z.; Su, J.; Zhao, Y.H.; Ryan, D.; Wu, H.K. Fabrication of a microfluidic $\mathrm{Ag} / \mathrm{AgCl}$ reference electrode and its application for portable and disposable electrochemical microchips. Electrophoresis 2010, 31, 3083-3089.

32. Polk, B.J.; Stelzenmuller, A.; Mijares, G.; MacCrehan, W.; Gaitan, M. Ag/AgCl microelectrodes with improved stability for microfluidics. Sens. Actuator B Chem. 2006, 114, 239-247.

33. Da Silva, E.T.S.G.; Miserere, S.; Kubota, L.T.; Merkoci, A. Simple on-plastic/paper inkjet-printed solid-state $\mathrm{Ag} / \mathrm{AgCl}$ pseudoreference electrode. Anal. Chem. 2014, 86, 10531-10534.

34. Desmond, D.; Lane, B.; Alderman, J.; Glennon, J.D.; Diamond, D.; Arrigan, D.W.M. Evaluation of miniaturised solid state reference electrodes on a silicon based component. Sens. Actuator B Chem. 1997, 44, 389-396.

35. Suzuki, H.; Shiroishi, H.; Sasaki, S.; Karube, I. Microfabricated liquid junction $\mathrm{Ag} / \mathrm{AgCl}$ reference electrode and its application to a one-chip potentiometric sensor. Anal. Chem. 1999, 71, 5069-5075.

36. Suzuki, H. Advances in the microfabrication of electrochemical sensors and systems. Electroanalysis 2000, 12, 703-715.

37. Du, J.L.; Chen, Z.F.; Chen, C.C.; Meyer, T.J. A half-reaction alternative to water oxidation: Chloride oxidation to chlorine catalyzed by silver ion. J. Am. Chem. Soc. 2015, 137, 3193-3196.

38. Fritz, J.J. Thermodynamic properties of chloro-complexes of silver-chloride in aqueous-solution. J. Solut. Chem. 1985, 14, 865-879.

39. Da Silva, R.A.B.; de Almeida, E.G.N.; Rabelo, A.C.; da Silva, A.T.C.; Ferreira, L.F.; Richter, E.M. Three electrode electrochemical microfluidic cell: Construction and characterization. J. Braz. Chem. Soc. 2009, 20, 1235-1241.

40. Simonis, A.; Dawgul, M.; Luth, H.; Schoning, M.J. Miniaturised reference electrodes for field-effect sensors compatible to silicon chip technology. Electrochim. Acta 2005, 51, 930-937.

41. Franklin, R.K.; Johnson, M.D.; Scott, K.A.; Shim, J.H.; Nam, H.; Kipke, D.R.; Brown, R.B. Iridium oxide reference electrodes for neurochemical sensing with MEMS 
microelectrode arrays. In Proceedings of IEEE Sensors 2005, Irvine, CA, USA, 31 October-3 November 2005; pp. 1400-1403.

42. Beati, A.A.G.F.; Reis, R.M.; Rocha, R.S.; Lanza, M.R.V. Development and evaluation of a pseudoreference $\mathrm{Pt} / \mathrm{Ag} / \mathrm{AgCl}$ electrode for electrochemical systems. Ind. Eng. Chem. Res. 2012, 51, 5367-5371.

43. Kasem, K.K.; Jones, S. Platinum as a reference electrode in electrochemical measurements. Platin. Met. Rev. 2008, 52, 100-106.

44. Dacuna, B.; Zaragoza, G.; Blanco, M.C.; Quintela, A.L.; Mira, J.; Rivas, J. Electrochemical synthesis of $\mathrm{Fe} / \mathrm{Ag}$ and $\mathrm{Co} / \mathrm{Ag}$ granular thin films. Mater. Sci. Forum 1998, 269-272, 307-312.

45. Yang, H.S.; Kang, S.K.; Choi, C.A.; Kim, H.; Shin, D.H.; Kim, Y.S.; Kim, Y.T. An iridium oxide reference electrode for use in microfabricated biosensors and biochips. Lab Chip 2004, 4, 42-46.

46. Xiong, J.W.; White, H.S. The I-V response of an electrochemical cell comprising two polarizable microelectrodes and the influence of impurities on the cell response. J. Electroanal. Chem. 2013, 688, 354-359.

47. Sarkar, S.; Mathwig, K.; Kang, S.; Nieuwenhuis, A.F.; Lemay, S.G. Redox cycling without reference electrodes. Analyst 2014, 139, 6052-6057.

48. Oja, S.M.; Zhang, B. Imaging transient formation of diffusion layers with fluorescence-enabled electrochemical microscopy. Anal. Chem. 2014, 86, 12299-12307.

49. Bradley, J.C.; Chen, H.M.; Crawford, J.; Eckert, J.; Ernazarova, K.; Kurzeja, T.; Lin, M.D.; McGee, M.; Nadler, W.; Stephens, S.G. Creating electrical contacts between metal particles using directed electrochemical growth. Nature 1997, 389, 268-271.

50. Loget, G.; Li, G.Z.; Fabre, B. Logic gates operated by bipolar photoelectrochemical water splitting. Chem. Commun. 2015, 51, 11115-11118.

51. Loget, G.; Zigah, D.; Bouffier, L.; Sojic, N.; Kuhn, A. Bipolar electrochemistry: From materials science to motion and beyond. Acc. Chem. Res. 2013, 46, 2513-2523.

52. Loget, G.; Roche, J.; Gianessi, E.; Bouffier, L.; Kuhn, A. Indirect bipolar electrodeposition. J. Am. Chem. Soc. 2012, 134, 20033-20036.

53. Loget, G.; Kuhn, A. Bipolar electrochemistry for cargo-lifting in fluid channels. Lab Chip 2012, 12, 1967-1971.

54. Fattah, Z.; Loget, G.; Lapeyre, V.; Garrigue, P.; Warakulwit, C.; Limtrakul, J.; Bouffier, L.; Kuhn, A. Straightforward single-step generation of microswimmers by bipolar electrochemistry. Electrochim. Acta 2011, 56, 10562-10566.

55. Loget, G.; Kuhn, A. Propulsion of microobjects by dynamic bipolar selfregeneration. J. Am. Chem. Soc. 2010, 132, 15918-15919.

56. Scida, K.; Sheridan, E.; Crooks, R.M. Electrochemically-gated delivery of analyte bands in microfluidic devices using bipolar electrodes. Lab Chip 2013, 13, 22922299. 
57. Chang, B.Y.; Chow, K.F.; Crooks, J.A.; Mavre, F.; Crooks, R.M. Two-channel microelectrochemical bipolar electrode sensor array. Analyst 2012, 137, 28272833.

58. Sheridan, E.; Hlushkou, D.; Anand, R.K.; Laws, D.R.; Tallarek, U.; Crooks, R.M. Label-free electrochemical monitoring of concentration enrichment during bipolar electrode focusing. Anal. Chem. 2011, 83, 6746-6753.

59. Dumitrescu, I.; Anand, R.K.; Fosdick, S.E.; Crooks, R.M. Pressure-driven bipolar electrochemistry. J. Am. Chem. Soc. 2011, 133, 4687-4689.

60. Sequeira, C.A.C.; Cardoso, D.S.P.; Gameiro, M.L.F. Bipolar electrochemistry, a focal point of future research. Chem. Eng. Commun. 2016, 203, 1001-1008.

61. Wang, Y.; Hernandez, R.M.; Bartlett, D.J.; Bingham, J.M.; Kline, T.R.; Sen, A.; Mallouk, T.E. Bipolar electrochemical mechanism for the propulsion of catalytic nanomotors in hydrogen peroxide solutions. Langmuir 2006, 22, 10451-10456.

62. Kline, T.R.; Paxton, W.F.; Mallouk, T.E.; Sen, A. Catalytic nanomotors: Remotecontrolled autonomous movement of striped metallic nanorods. Angew. Chem. Int. Ed. 2005, 44, 744-746.

63. Paxton, W.F.; Kistler, K.C.; Olmeda, C.C.; Sen, A.; St Angelo, S.K.; Cao, Y.Y.; Mallouk, T.E.; Lammert, P.E.; Crespi, V.H. Catalytic nanomotors: Autonomous movement of striped nanorods. J. Am. Chem. Soc. 2004, 126, 13424-13431.

64. Ma, C.X.; Zaino, L.P.; Bohn, P.W. Self-induced redox cycling coupled luminescence on nanopore recessed disk-multiscale bipolar electrodes. Chem. Sci. 2015, 6, 3173-3179.

65. Oleinick, A.I.; Battistel, D.; Daniele, S.; Svir, I.; Amatore, C. Simple and clear evidence for positive feedback limitation by bipolar behavior during scanning electrochemical microscopy of unbiased conductors. Anal. Chem. 2011, 83, 4887-4893.

66. Richter, M.M. Electrochemiluminescence (ECL). Chem. Rev. 2004, 104, 30033036.

67. Zevenbergen, M.A.G.; Wolfrum, B.L.; Goluch, E.D.; Singh, P.S.; Lemay, S.G. Fast electron-transfer kinetics probed in nanofluidic channels. J. Am. Chem. Soc. 2009, 131, 11471-11477.

68. Arora, A.; Eijkel, J.C.T.; Morf, W.E.; Manz, A. A wireless electrochemiluminescence detector applied to direct and indirect detection for electrophoresis on a microfabricated glass device. Anal. Chem. 2001, 73, 56335633.

69. Leinweber, F.C.; Eijkel, J.C.T.; Bower, J.G.; van den Berg, A. Continuous flow microfluidic demixing of electrolytes by induced charge electrokinetics in structured electrode arrays. Anal. Chem. 2006, 78, 1425-1434.

70. Bakker, E.; Pretsch, E. Modern potentiometry. Angew. Chem. Int. Ed. 2007, 46, 5660-5668.

71. Bakker, E. Dynamic potentiometry: New concepts for ion-selective electrodes. Abstr. Pap. Am. Chem. Soc. 2005, 230, U300-U300. 
72. Bakker, E.; Pretsch, E. Nanoscale potentiometry. Trends Anal. Chem 2008, 27, 612-618.

73. Pungor, E.; Toth, K. Ion-selective membrane electrodes-A review. Analyst 1970, 95, 625-648.

74. Singh, P.S. From sensors to systems: CMOS-integrated electrocheimcal biosensors. IEEE Access 2015, 3, 249-259.

75. Zhu, X.S.; Choi, J.W.; Ahn, C.H. A new dynamic electrochemical transduction mechanism for interdigitated array microelectrodes. Lab Chip 2004, 4, 581-587.

76. Zhu, X.S.; Ahn, C.H. Electrochemical determination of reversible redox species at interdigitated array micro/nanoelectrodes using charge injection method. IEEE Trans. Nanobiosci. 2005, 4, 164-169.

77. Zhu, X.S.; Ahn, C.H. On-chip electrochemical analysis system using nanoelectrodes and bioelectronic CMOS chip. IEEE Sens. J. 2006, 6, 1280-1286. 


\section{Redox cycling without reference electrode}

The reference electrode is a key component in electrochemical measurements, yet it remains a challenge to implement a reliable reference electrode in miniaturized electrochemical sensors. Here we explore experimentally and theoretically an alternative approach based on redox cycling which eliminates the reference electrode altogether. We show that shifts in the solution potential caused by the lack of reference can be understood quantitatively, and determine the requirements for accurate measurements in miniaturized systems in the absence of a reference electrode.

The contents of this chapter are published as Sahana Sarkar, Klaus Mathwig, Shuo Kang, Ab F. Nieuwenhuis and Serge G. Lemay, Redox Cycling without Reference Electrodes, Analyst, 2014, 139, 6052-6057. 


\subsection{Introduction}

Motivated by the wide applications of electrochemical sensors in the biomedical domain, there is continuously rising interest in miniaturised assays suitable for integration. Electrochemical sensors are particularly well suited for cost effective realization and integration with fluidics due to their inherent compatibility with microfabrication. ${ }^{1-11}$ Irrespective of the particular measurement method employed (amperometry, potentiometry, impedance spectroscopy, etc.), the reference electrode is a key component of electrochemical sensors. In miniaturized systems, however, the volume-to-surface area ratio of reference electrodes decreases, thereby reducing their lifetime and stability. ${ }^{12-16}$ For example, due to downscaling, dissolution of the electrode material, leakage of the reference solution and contamination at the junction of salt bridges become difficult to avoid, rendering many sensors short-lived or unreliable. Realizing lab-on-a-chip devices without the use of conventional reference electrodes ${ }^{12,14-17}$ could therefore significantly improve the performance and usability of miniaturized electrochemical sensors.

One potential route for bypassing the reference electrode is offered by systems based on redox cycling, in which a current is generated by successively reducing and oxidizing an analyte at two independently biased electrodes. While the primary purpose of redox cycling is typically to increase the magnitude of the electrochemical current or to improve selectivity, ${ }^{18-20}$ it also provides a pathway for electrochemically generated currents to flow through the analyte solution without the need for a reference or auxiliary electrode. In this work we explore the underlying principles of operation of redox cycling systems without a reference electrode. As a prototypical system we focus on fluidic nanogap sensors, sketched in Figure 3.1a, consisting of two closely spaced parallel electrodes separated by a few tens of nanometers and forming the floor and roof of a fluid-filled nanochannel. Electrochemically active molecules undergo successive redox cycling by diffusing back and forth between these electrodes, generating a current that is proportional to the analyte concentration. The cross sectional diagram and top views of such a device are shown in Figure 3.1b and 3.1c, respectively. An example application of a variant of this device in which operation without a reference electrode would be necessary is a sealable chamber allowing locally generated analyte molecules (e.g., by a catalytic reaction) to be collected and detected in a fL-level volume (Figure 3.1d). Another example is the incorporation of a nanogap directly in (or, more practically, in a side channel of) 
a capillary flow detection system (Figure3.1e). Other configurations where the same general principles apply include interdigitated arrays ${ }^{19}, 21-24$ and ring-disk electrodes. ${ }^{25-26}$

We emphasize that this work does not in any way diminish the relevance of previous efforts at realizing miniaturized reference electrodes, but instead provides an alternative, complementary approach.

a)

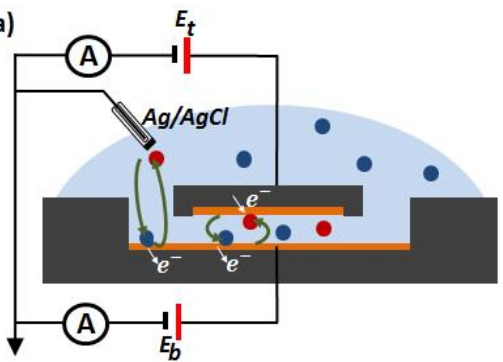

c)

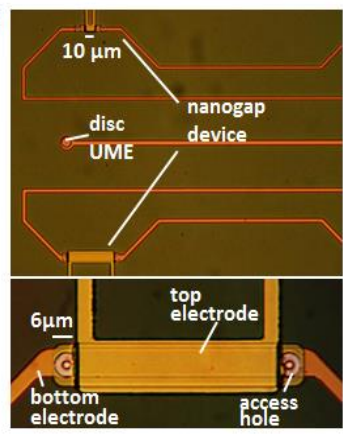

b)

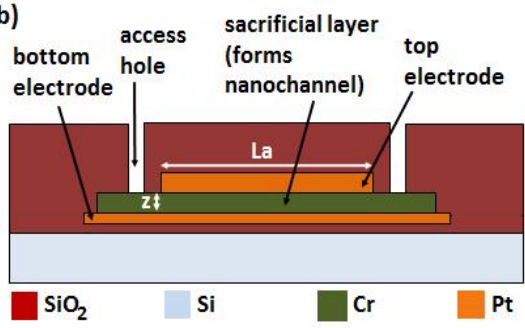

d)

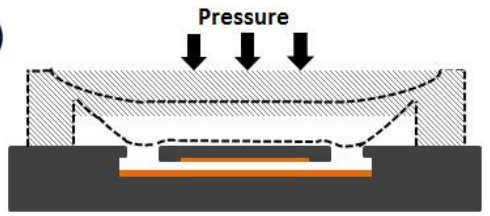

e)

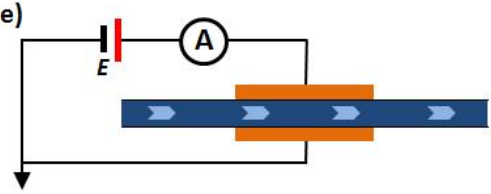

Figure 3.1. a) Nanogap sensor in the conventional 3-electrode configuration that includes a reference electrode. Redox molecules undergo successive oxidation and reduction reactions at the two planar electrodes while diffusing within the nanochannel. An ionic current also flows to the reference electrode. b) Schematic diagram of the cross section of the device prior to etching (not drawn to scale). $L_{a}$ and $z$ represent the active (or overlapping) length of the top and bottom electrodes and the height of the nanogap, respectively. c) Optical microscopic image of the top view of the device before the sacrificial layer is etched. d) Nanogap sensor with an integrated microfluidic valve (PDMS). In the closed state the reference electrode (not shown) gets disconnected, as represented by the dashed lines. e) Capillary based fluid flow system consisting of two electrodes imbedded in a flow through (nano) channel. 


\subsection{Materials and methods}

A nanogap sensor with two parallel planar electrodes, as shown in Figure 3.1a-c, was used in our experiments. The method for micro fabricating the devices was similar to that reported previously. ${ }^{27}$ In brief, the device consisted of a sacrificial chromium layer (thickness $z=60 \pm 5 \mathrm{~nm}$ ) deposited between two platinum electrodes. The areas of the top and bottom electrodes were $60 \mu \mathrm{m}^{2}$ and $102 \mu \mathrm{m}^{2}$, respectively, whereas the overlapping area between the two electrodes where redox cycling took place was $30 \mathrm{\mu m}^{2}$. Prior to the measurements, chromium etchant (BASF, Chromium Etch Selectipur) was filled through the inlets in order to etch the sacrificial layer and create the nanochannel. The electrodes were cleaned before conducting the measurements by cycling their potential in $5 \mathrm{mM} \mathrm{H}_{2} \mathrm{SO}_{4}$. A polydimethylsiloxane (PDMS) reservoir filled with analyte solution was positioned above the device and a standard $\mathrm{Ag} / \mathrm{AgCl}$ electrode (BASi, MF 2079, RE-5B) inserted in this reservoir was used as reference electrode. The reference electrode was thus located outside the nanochannel in the reservoir. No auxiliary electrode was necessary as the current through the reference electrode remained well below $1 \mathrm{nA}$, as shown in detail below (Figure 3.2). All solutions were prepared in $18 \mathrm{M} \Omega \mathrm{cm}$ Milli-Q water (Millipore). Ferrocenedimethanol $\left(\mathrm{Fc}(\mathrm{MeOH})_{2}\right.$, Sigma-Aldrich, cat. no- 372625, diffusion coefficient $D=6.7 \times 10^{-10} \mathrm{~m}^{2} / \mathrm{s}$ ) was chosen as a simple prototypical redox species and was prepared as a $1 \mathrm{mM}$ solution reduced $\mathrm{Fc}(\mathrm{MeOH})_{2}$ with $0.1 \mathrm{M} \mathrm{KCl}$ (Sigma-Aldrich, cat. no- P3911) added as supporting electrolyte.

In order to investigate the role of the reference electrode in redox cycling measurements, experiments were conducted with two complementary electrode configurations. In the first configuration the $\mathrm{Ag} / \mathrm{AgCl}$ electrode was held at a fixed bias, thus serving as a proper reference electrode, while the current flowing through it was monitored using a transimpedance amplifier (Femto, DDPCA-300). In the second scheme, there was no true reference electrode present. The standard $\mathrm{Ag} / \mathrm{AgCl}$ reference electrode instead served as a non-invasive probe to the solution potential, $E_{\text {sol. }}$. A Keithley 617 electrometer was used for these potentiometric measurements. The chassis ground and common (COM) terminals of all the instruments were connected together throughout the measurements; we refer to this potential as the circuit reference potential (or ground for short). Scan rates were kept sufficiently low such that steady-state mass transport conditions were achieved. 


\subsection{Experimental results}

We first concentrate on measurements conducted with an active reference electrode. Figure 3.2a shows the cyclic voltammogram obtained at the bottom electrode when its potential, $E_{b}$, was varied from 0 to $0.5 \mathrm{~V}$ while the potential of the top electrode, $E_{t}$, was disconnected (black curve). A sigmoidal cyclic voltammogram was observed with a wave height (difference between the plateau currents at $E_{t}=0$ $\mathrm{V}$ and $E_{t}=0.5 \mathrm{~V}$ ) of $i_{\mathrm{im}}=305 \mathrm{pA}$. The cyclic voltammogram simultaneously obtained at the reference electrode is also shown (green curve); this current has the same magnitude but an opposite sign to that measured at the bottom electrode, demonstrating that all current from the working electrode flows to the reference electrode and therefore that there are no significant parasitic current leakage pathways in the system. The voltammogram (obtained at the bottom electrode) is shifted downward by $52 \mathrm{pA}$ compared to that expected for the original solution of $\mathrm{Fc}(\mathrm{MeOH})_{2}$ in the reduced form. This indicates that this solution became partly oxidized after exposure to the reservoir, consistent with previous observations ${ }^{28}$ and as verified explicitly by re-extracting the solution and analysing it ex-situ (see Appendix A).

Figure $3.2 \mathrm{~b}$ shows that sweeping $E_{t}$ while keeping $E_{b}$ floating gave rise to an essentially identical cyclic voltammogram. While this may appear counterintuitive since the top electrode is located further from the access holes, and thus one might expect smaller currents, this effect can be understood by noting that the bottom electrode must necessarily adjust its potential to that of the top electrode because of the strong coupling introduced by redox cycling, as discussed earlier. ${ }^{28}$ Similarly, measurements where both $E_{t}$ and $E_{b}$ were swept simultaneously (data not shown) yielded once again the same voltammogram. In all the above voltammograms, the potential at which the obtained current crosses the zero-current axis - that is, the rest potential, $V_{r p}$ - was found to be $0.21 \mathrm{~V}$.

Figure 3.2c illustrates a typical voltammogram obtained during redox cycling with a reference electrode and $E_{b}$ swept between 0 and $0.5 \mathrm{~V}$ while $E_{t}$ was kept biased at 0 $V$. An oxidising current at the bottom electrode giving ilim of $30 \mathrm{nA}$ was observed. An equal and opposite current was observed at the top (reducing) electrode, as expected for redox cycling. The amplification factor for redox cycling can be defined as the ratio of $i_{\lim }$ at the electrode during redox cycling to that of the current at the reference electrode (Figure 3.2a and 3.2c), yielding an amplification factor of $\sim 98$. It is however crucial to note that a finite current still flows through the reference electrode during 
a)

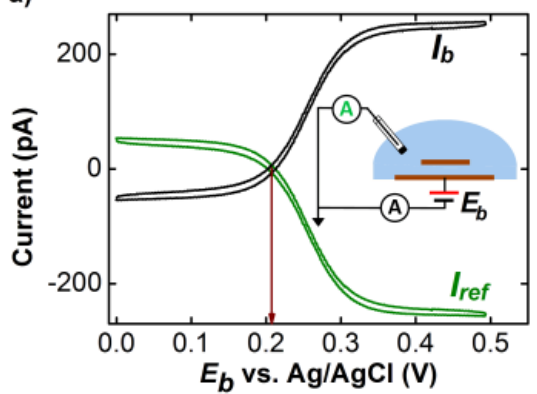

c)

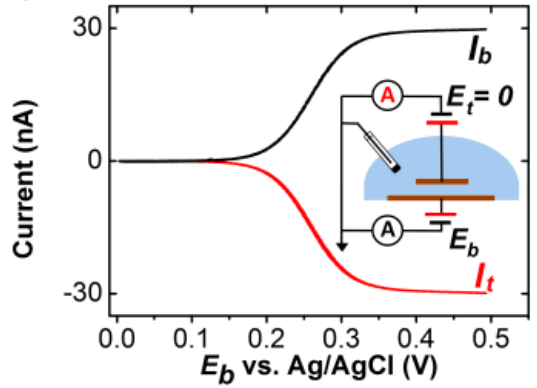

e)

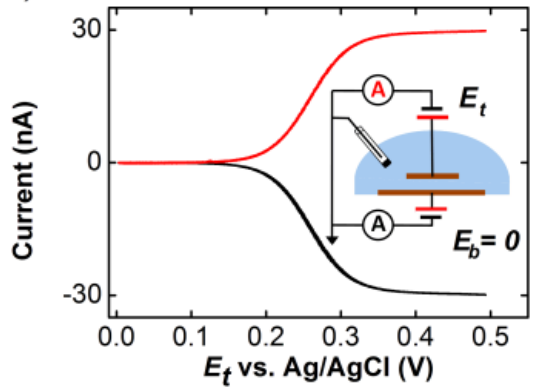

b)

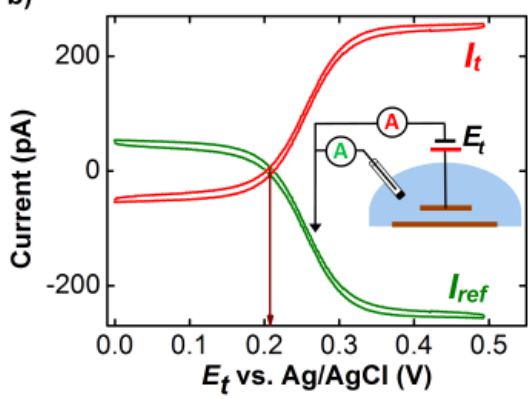

d)

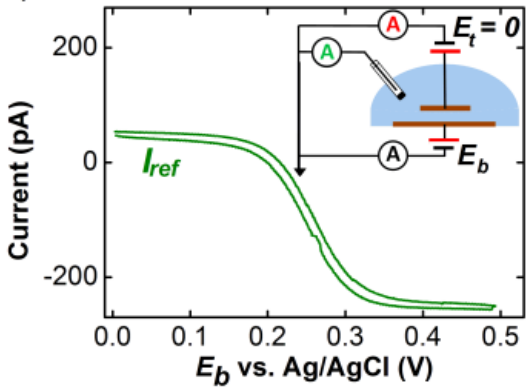

f)

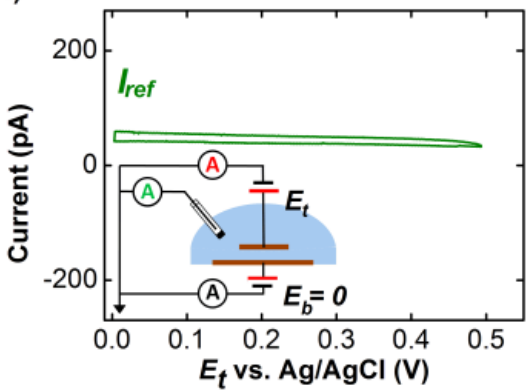

Figure 3.2. Cyclic voltammograms for $1 \mathrm{mM} \mathrm{Fc}(\mathrm{MeOH})_{2}$ in $0.1 \mathrm{M} \mathrm{KCl}$ at a scan rate of 5 $\mathrm{mV} / \mathrm{s}$. The black, red and green lines represent the currents measured at the top, bottom and reference electrodes, respectively. a) The bottom electrode $\left(E_{b}\right)$ was swept while the top electrode was left floating; the rest potential was $V_{r p}=0.21 \mathrm{~V}$. b) The top electrode $\left(E_{t}\right)$ was swept while the bottom electrode was left floating; again $V_{r p}=0.21$ $\checkmark$. c) $E_{b}$ was swept while Et was biased at $0 \mathrm{~V}$ giving rise to a redox cycling current of 30 nA. d) The current through the reference electrode during redox cycling, Iref, was essentially identical to that observed in cases (a) and (b). e) Et was swept while $E_{b}$ was biased at $0 \mathrm{~V}$. f) Iref measured simultaneously with the voltammogram in panel (e). The current is nearly constant, in sharp contrast with panel (d).

redox cycling, as explicitly shown in Figure 3.2d. This current originates from 
molecules from the bulk that diffused through the access holes into the channel, reacted at the bottom electrode and returned once again to the bulk reservoir. Correspondingly, the values of /ref observed in Figure 3.2d are essentially equal to those obtained in the case of Figure 3.2a. Figure 3.2e shows that when $E_{t}$ was varied while $E_{b}$ was biased at $0 \mathrm{~V}$, oxidizing and reducing currents were now observed at the top and bottom electrodes, respectively, as expected since the role of the electrodes was reversed. In this case, however, the current through the reference electrode (Figure 3.2f) was roughly constant (apart from slight drift and hysteresis) with a value close to the $E_{b}=0 \mathrm{~V}$ values of $50 \mathrm{pA}$ observed in Figure 3.2a and 3.2c. This reflects

a)

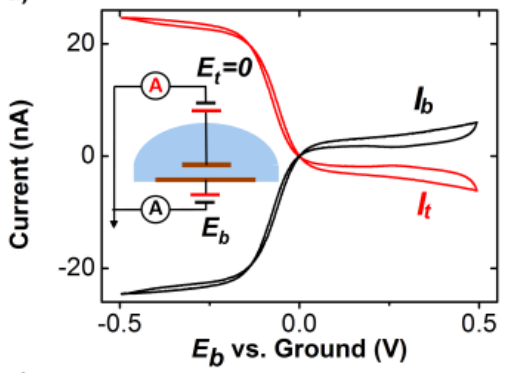

c)

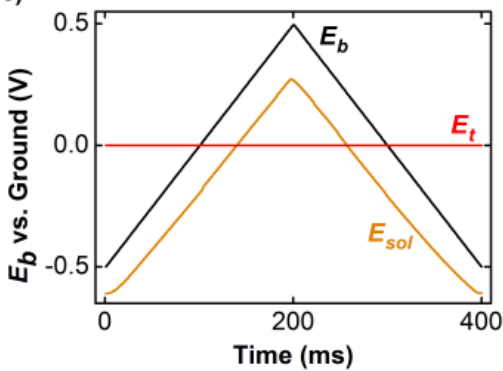

b)

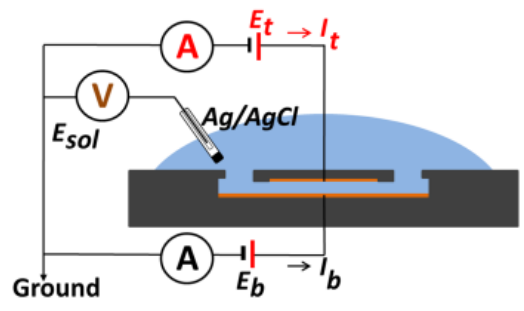

d)

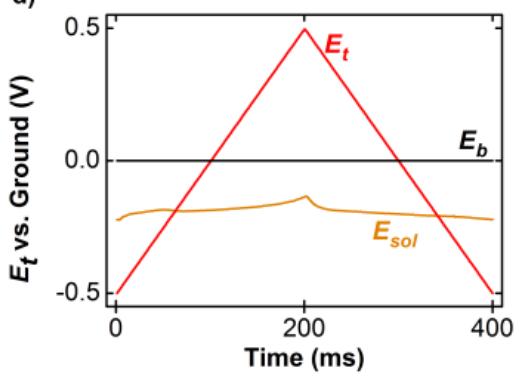

Figure 3.3. Cyclic voltammograms for $1 \mathrm{mM} \mathrm{Fc}(\mathrm{MeOH})_{2}$ in $0.1 \mathrm{M} \mathrm{KCl}$ at a scan rate of 5 $\mathrm{mV} / \mathrm{s}$ in the absence of the reference electrode. a) Currents obtained at the top (black) and bottom (red) electrodes when the bottom electrode $\left(E_{b}\right)$ was swept while the top electrode $\left(E_{t}\right)$ was biased at $0 \mathrm{~V}$ with respect to the circuit reference potential. b) Schematic diagram of the setup configuration; the $\mathrm{Ag} / \mathrm{AgCl}$ electrode now serves as a probe of the solution potential, $E_{\text {sol. }}$. All potentials are referred to the external circuit potential, here labelled as Ground. c) $E_{b}$ was varied (black) while $E_{t}$ was grounded (red) and $E_{\text {sol }}$ was measured (orange). $E_{\text {sol }}$ closely follows the potential of the sweeping electrode, $E_{b}$. d) $E_{t}$ was varied while $E_{b}$ was grounded. $E_{\text {sol }}$ experiences only minor variations compared to the sweeping potential, $E_{t}$. 
the fact that mass transport to the reference electrode is much more efficient from the bottom than from the top electrode due to the channel geometry: the bottom electrode is directly exposed to the access holes, whereas molecules leaving the top electrode interact with near-certainty with the bottom electrode on their way to the bulk. As a result, the potential of the top electrode hardly influences / ref.

We now turn to measurements done in the second configuration, in which no active reference electrode was used and the potential of the solution was allowed to float. Figure 3.3 a shows a cyclic voltammogram wherein $E_{t}$ was biased at $0 \mathrm{~V}$ while $E_{b}$ was cycled between -0.5 and $0.5 \mathrm{~V}$ with respect to the circuit reference potential. These curves are qualitatively different from those observed when the reference was connected (Figure 3.2c), with both electrodes exhibiting reducing and oxidizing currents depending on the polarity of $E_{b}$. Thereafter, we introduced an $\mathrm{Ag} / \mathrm{AgCl}$ electrode to monitor the potential of the floating bulk electrolyte with respect to the circuit reference potential, as shown in Figure 3.3b.

Figure $3.3 \mathrm{c}$ shows the corresponding measured potential of the solution, $E_{\text {sol }}$ (orange curve) while cycling $E_{b}$ (black curve) and keeping $E_{t}$ equal to $0 \mathrm{~V}$ (red curve). $E_{\text {sol }}$ follows the applied potential quite closely. Similarly, Figure 3.3d shows the complementary case where $E_{t}$ was cycled. In this case, sweeping the potential had a negligible effect on the bulk solution. These results illustrate qualitatively that the more effective mass transport from the bottom electrode to the bulk solution observed in Figure 3.2 directly translate into a stronger influence of the bottom electrode on the solution potential.

\subsection{Analysis}

Using the measured potential of the solution, $E_{\text {sol, }}$ and the known applied potentials, $E_{t}$ and $E_{b}$ (all referred to the external circuit potential), we can reconstruct the potential differences across the solid-liquid interfaces at both electrodes, $E_{\mathrm{t}, \mathrm{sol}}$ and $E_{b, s o l}$. These are the preferred variables for describing electrochemical processes since it is these potential differences that drive electrochemical reactions. In terms of the directly measurable quantities defined above, these potentials are simply given by

$$
E_{\mathrm{t} / \mathrm{b}, \mathrm{sol}}=E_{t / b}-E_{\mathrm{sol}}
$$


The potentials of Figure $3.3 \mathrm{c}$ and $3.3 \mathrm{~d}$ re-expressed in terms of $E_{\mathrm{t}, \mathrm{sol}}$ and $E_{\mathrm{b}, \text { sol }}$ are shown in Figure 3.4a and 3.4b, respectively. In Figure 3.4a, it is observed that although $E_{b}$ was swept over a broad range of potentials, $E_{\mathrm{b}, \text { sol }}$ remained relatively constant near the $V_{r p}$ at $0.21 \mathrm{~V}$. On the contrary, $E_{\mathrm{t}, \text { sol }}$ changed from $0.6 \mathrm{~V}$ to $-0.27 \mathrm{~V}$, thereby showing that the top electrode was effectively being swept during the measurement while the bottom electrode held a nearly constant potential with respect to the solution. Figure $3.4 \mathrm{~b}$ exhibits exactly the same behavior except that the potential curves are reflected through the $E_{t / b}=0 \mathrm{~V}$ axis. While this may appear to contrast with the large differences observed between Figure $3.3 \mathrm{c}$ and $3.3 \mathrm{~d}$, this mirror symmetry must necessarily be present since, in the case where the solution potential is allowed to float, only the potential difference, $E_{t}-E_{b}$, can be relevant in establishing the interfacial potentials. Both electrode configurations thus lead to the same situation wherein the bottom electrode remains at a nearly constant potential while the top electrode experiences an effective potential sweep that is applied as a potential difference between the two electrodes.

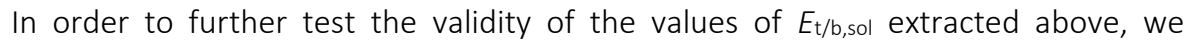
attempted to reconstitute the cyclic voltammograms of Figure 3.3a using the potentials determined in Figure $3.4 \mathrm{a}$ and $3.4 \mathrm{~b}$. The shape of redox-cycling voltammograms in nanogap devices has previously been shown to be well described by the Butler-Volmer formalism, ${ }^{28}$

$$
I_{t}^{r c}=-I_{b}^{r c}=i_{\lim } \frac{\frac{1}{1+\mathrm{e}^{-f \eta_{t}}}-\frac{1}{1+\mathrm{e}^{-f \eta_{b}}}}{1+\frac{D}{k_{0} z}\left(\frac{\mathrm{e}^{\alpha f \eta_{t}}}{1+\mathrm{e}^{f \eta_{t}}}+\frac{\mathrm{e}^{\alpha f \eta_{b}}}{1+\mathrm{e}^{f \eta_{b}}}\right)}
$$

Here $z$ is the height of the nanochannel, $f=F / R T, F$ is the Faraday constant, $R$ is the gas constant, $T$ is the absolute temperature and $\eta_{t, b}$ are the overpotentials applied to the top and bottom electrodes, respectively. That is, $\eta_{t / b}=E_{\mathrm{t} / \mathrm{b} \text {,sol }}-E_{f}$, where $E_{f}$ is the formal potential of the reaction. $E_{f}$ was measured directly using the same solution with a platinum UME (BASi, MF 2005) and the same reference electrode as used during the other measurements and was found to be $0.26 \mathrm{~V}$. ilim obtained during the redox cycling (30 nA, Figure 3.2c,e) was directly substituted in Equation 3.2. The calculated value of $i_{\mathrm{im}}$ is marginally higher $(\sim 32 \mathrm{nA})$ and this difference can be attributed to the buckling of the electrodes due to stress (resulting from the passivation) that leads to an increase in the height of the channel. ${ }^{29}$ The remaining unknown parameters, i.e., the transfer coefficient, $\alpha$, and the heterogeneous rate 
constant, $k_{0}$, were found to be 0.50 and $0.06 \mathrm{~m} / \mathrm{s}$, respectively, consistent with previous measurements. ${ }^{28}$

Substituting the measured interfacial potentials from Figure 3.4a and 3.4b into Equation 3.2 yields the calculated cyclic voltammograms (green curves) plotted in Figure 3.4c and 3.4d, respectively. These are in excellent agreement with the measured currents at the bottom (black curve) and top (red curve) electrodes obtained when $E_{b}$ and $E_{t}$ were swept, respectively.
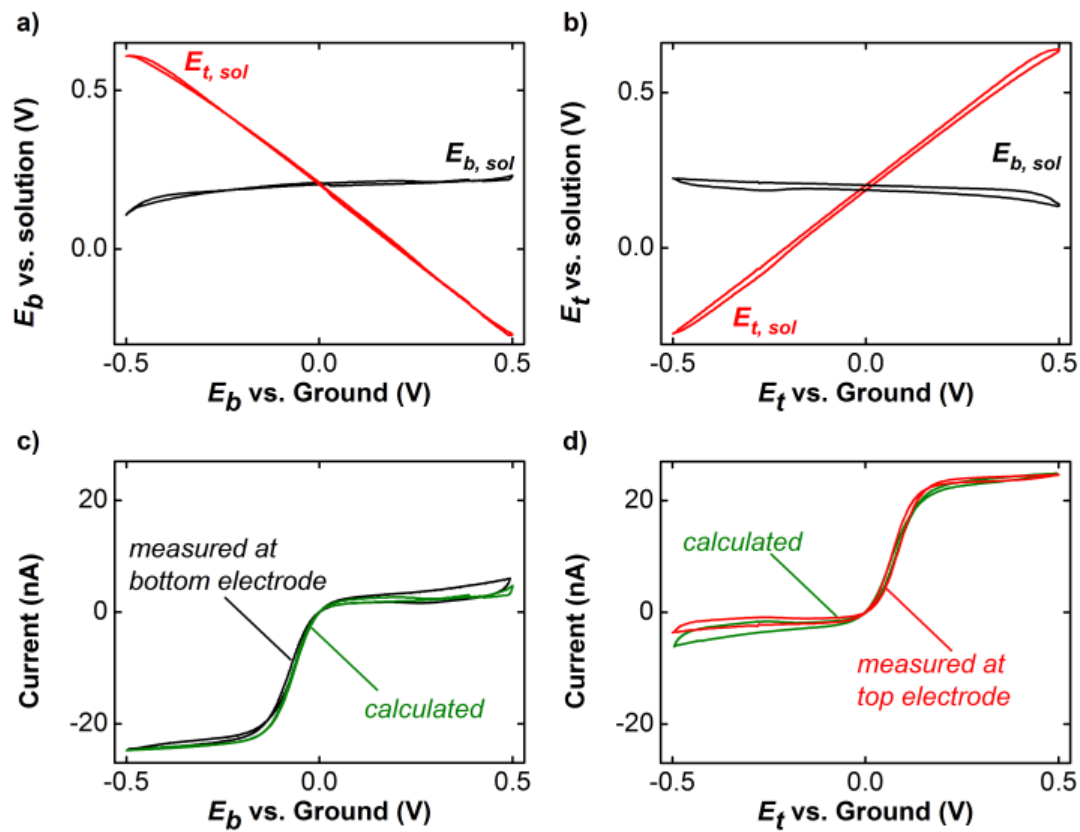

Figure 3.4. a) The potentials at the electrodes with respect to the solution, $E_{\mathrm{b}, \text { sol }}$ and $E_{\mathrm{t}, \text { sol, }}$ while sweeping $E_{b}$. $E_{\mathrm{b}, \text { sol }}$ remained relatively constant near a value of $V_{r p}=0.21 \mathrm{~V}$ while $E_{\mathrm{t}, \text { sol }}$ varied from $0.6 \mathrm{~V}$ to $-0.27 \mathrm{~V}$. b) Same as a) except that $E_{T}$ is swept. c) The expected current was calculated using Equation 3.2 and the potentials of panel a). The calculated and measured currents (represented as black and green lines, respectively) are in excellent agreement. d) Same as c) for the case where $E_{t}$ is swept (panel b)). The calculated and measured currents are represented as red and green lines, respectively.

\subsection{Discussion}

We first briefly summarize the situation for the classical system consisting of cyclic voltammetry at a UME. Within the operating window of the solvent, the detailed 
shape of the voltammogram depends on the solution composition and generically includes both a target analyte signal as well as parasitic currents resulting from reactions involving dissolved oxygen, hydronium ions and/or contaminants. Under typical steady-state mass transport conditions the current varies monotonically with electrode potential and vanishes at the rest potential, $V_{r p}$, as discussed before (Figure 3.2a,b). A floating working electrode starting at an arbitrary potential will reduce or oxidize molecules in solution and accumulate charge until its potential drifts to $V_{r p}$, and this potential can be measured with a high-impedance voltmeter (Figure 3.3). This description was further extended by Xiong and White to the case of a cell comprising of two polarizable electrodes in the absence of a reference electrode. ${ }^{30}$

The same principle applies to the redox-cycling case: in the absence of a reference electrode, the solution potential is determined by the condition such that no net current can be injected into the solution at steady state,

$$
I_{t}+I_{b}=0
$$

Here $I_{t}$ and $I_{b}$ are the currents at the top and bottom electrodes, respectively. That is, the solution potential will drift to the value at which Equation 3.3 is satisfied. In redox-cycling systems, the steady state current at each electrode can further be broken into two components: a redox-cycling current, $I_{t / b}^{r c}$, and an excess current, $I_{t / b}^{0}$. The latter includes all other current components such as parasitic reactions, irreversible reactions and reactions occurring in regions of the electrodes not implicated in redox cycling. This can be written as

$$
I_{t / b}=I_{t / b}^{0}+I_{t / b}^{r c}
$$

As was seen in Figure 3.2, the magnitude of $I_{t / b}^{r c}$ is far larger than $I_{t / b}^{0}$ and dominates the overall current levels during redox cycling. Noting that the redox-cycling current obtained at the two electrodes is, by definition, equal and opposite $\left(I_{t}^{r c}=-I_{b}^{r c}\right)$, however, Equation 3.1 and 3.2 reduce to the more specific condition

$$
I_{t}^{0}+I_{b}^{0}=0
$$

That is, in the absence of a reference electrode, the potential of the electrolyte solution is fully determined by the excess current, $I_{t, b}^{0}$, even though it represents only a small fraction of the total currents flowing through the electrodes. This situation is reminiscent of positive-feedback scanning electrochemistry (SECM) measurements on a floating substrate, ${ }^{31-36}$ but here it is the solution itself that adjusts its potential with respect to the electrodes. 


\subsection{Conclusion}

Redox cycling measurements without a well-defined reference electrode are a priori difficult to interpret because it is not known how a potential difference applied between the two working electrodes is divided between them. We have argued that determining this potential is further complicated by the fact that it is fully determined by "excess" background currents rather than by the (usually much larger) redox cycling current. On the other hand, we have shown that the geometry of the redox cycling device can be exploited to alleviate this difficulty to a significant extent. In situations where one of the redox cycling electrodes has a much more significant exposure to the sample solution, this electrode can readily pin the solution potential to a nearly constant value, allowing the potential of the second electrode with respect to the solution to be controlled. While we have concentrated here on nanogap electrodes embedded in nanochannels, the same principles can be applied to other asymmetric redox cycling geometries such as recessed ring-disk electrodes or even interdigitated electrodes if one of the electrodes is recessed with respect to the other.

\subsection{References}

1. Dutse, S.W.; Yusof, N.A., Microfluidics-Based Lab-on-Chip Systems in DNA-Based Biosensing: An Overview. Sensors-Basel 2011, 11, 5754-5768.

2. Lee, T.M.H.; Carles, M.C.; Hsing, I.M., Microfabricated PCR-electrochemical device for simultaneous DNA amplification and detection. Lab Chip 2003, 3, 100105.

3. Webster, T.A.; Goluch, E.D., Electrochemical detection of pyocyanin in nanochannels with integrated palladium hydride reference electrodes. Lab Chip 2012, 12, 5195-5201.

4. Fragoso, A., et al., Integrated microfluidic platform for the electrochemical detection of breast cancer markers in patient serum samples. Lab Chip 2011, 11, 625-631.

5. Goral, V.N.; Zaytseva, N.V.; Baeumner, A.J., Electrochemical microfluidic biosensor for the detection of nucleic acid sequences. Lab Chip 2006, 6, 414-421.

6. Koudelka-Hep, M.; van der Wal, P.D., Microelectrode sensors for biomedical and environmental applications. Electrochim Acta 2000, 45, 2437-2441.

7. Kraly, J.R.; Holcomb, R.E.; Guan, Q.; Henry, C.S., Review: Microfluidic applications in metabolomics and metabolic profiling. Anal Chim Acta 2009, 653, 23-35.

8. Lin, Y.H.; Matson, D.W.; Bennett, W.D.; Thrall, K.D.; Timchalk, C., Integrated microfluidics/electrochemical sensor system for field-monitoring of toxic metals. Microreaction Technology: Industrial Prospects 2000, 588-596. 
9. Wang, J., Electrochemical detection for capillary electrophoresis microchips: A review. Electroanalysis 2005, 17, 1133-1140.

10. Zou, Z.W.; Kai, J.H.; Rust, M.J.; Han, J.; Ahn, C.H., Functionalized nano interdigitated electrodes arrays on polymer with integrated microfluidics for direct bio-affinity sensing using impedimetric measurement. Sensors and Actuators a-Physical 2007, 136, 518-526.

11. Grayson, A.C.R.; Shawgo, R.S.; Li, Y.W.; Cima, M.J., Electronic MEMS for triggered delivery. Adv Drug Deliver Rev 2004, 56, 173-184.

12. Shinwari, M.W.; Zhitomirsky, D.; Deen, I.A.; Selvaganapathy, P.R.; Deen, M.J.; Landheer, D., Microfabricated Reference Electrodes and their Biosensing Applications. Sensors-Basel 2010, 10, 1679-1715.

13. Suzuki, H., Advances in the microfabrication of electrochemical sensors and systems. Electroanalysis 2000, 12, 703-715.

14. Schwarz, M.A.; Galliker, B.; Fluri, K.; Kappes, T.; Hauser, P.C., A two-electrode configuration for simplified amperometric detection in a microfabricated electrophoretic separation device. Analyst 2001, 126, 147-151.

15. Simonis, A.; Luth, H.; Wang, J.; Schoning, M.J., New concepts of miniaturised reference electrodes in silicon technology for potentiometric sensor systems. Sensor Actuat B-Chem 2004, 103, 429-435.

16. Franklin, R.K.; Johnson, M.D.; Scott, K.A.; Shim, J.H.; Nam, H.; Kipke, D.R.; Brown, R.B., Iridium oxide reference electrodes for neurochemical sensing with MEMS microelectrode arrays. leee Sensor 2005, 1400-1403.

17. Suzuki, H.; Shiroishi, H.; Sasaki, S.; Karube, I., Microfabricated liquid junction $\mathrm{Ag} / \mathrm{AgCl}$ reference electrode and its application to a one-chip potentiometric sensor. Anal Chem 1999, 71, 5069-5075.

18. Wolfrum, B.; Zevenbergen, M.; Lemay, S., Nanofluidic redox cycling amplification for the selective detection of catechol. Analytical chemistry 2008, 80, 972-977.

19. Goluch, E.D.; Wolfrum, B.; Singh, P.S.; Zevenbergen, M.A.G.; Lemay, S.G., Redox cycling in nanofluidic channels using interdigitated electrodes. Anal Bioanal Chem 2009, 394, 447-456.

20. Straver, M.G.; Odijk, M.; Olthuis, W.; van den Berg, A., A simple method to fabricate electrochemical sensor systems with predictable high-redox cycling amplification. Lab on a chip 2012, 12, 1548-1553.

21. Dam, V.A.T.; Olthuis, W.; van den Berg, A., Redox cycling with facing interdigitated array electrodes as a method for selective detection of redox species. Analyst 2007, 132, 365-370.

22. Heo, J.I.; Shim, D.S.; Teixidor, G.T.; Oh, S.; Madou, M.J.; Shin, H., Carbon Interdigitated Array Nanoelectrodes for Electrochemical Applications. J Electrochem Soc 2011, 158, J76-J80.

23. Niwa, O.; Xu, Y.; Halsall, H.B.; Heineman, W.R., Small-Volume Voltammetric Detection of 4-Aminophenol with Interdigitated Array Electrodes and Its Application to Electrochemical Enzyme-Immunoassay. Analytical chemistry 1993, 65, 1559-1563. 
24. Sanderson, D.G.; Anderson, L.B., Filar Electrodes - Steady-State Currents and Spectroelectrochemistry at Twin Interdigitated Electrodes. Analytical chemistry 1985,

25. Ma, C.X.; Contento, N.M.; Gibson, L.R.; Bohn, P.W., Redox Cycling in NanoscaleRecessed Ring-

26. Zhao, G.; Giolando, D.M.; Kirchhoff, J.R., Carbon Ring Disk Ultramicroelectrodes. Analytical chemistry 1995, 67, 1491-1495.

27. Kang, S.; Mathwig, K.; Lemay, S.G., Response time of nanofluidic electrochemical sensors. Lab Chip 2012, 12, 1262-1267.

28. Zevenbergen, M.A.G.; Wolfrum, B.L.; Goluch, E.D.; Singh, P.S.; Lemay, S.G., Fast Electron-Transfer Kinetics Probed in Nanofluidic Channels. Journal of the American Chemical Society 2009, 131, 11471-11477.

29. Zevenbergen, M.A.G.; Krapf, D.; Zuiddam, M.R.; Lemay, S.G., Mesoscopic concentration fluctuations in a fluidic nanocavity detected by redox cycling. Nano letters 2007, 7, 384-388.

30. Xiong, J.W.; White, H.S., The i-V response of an electrochemical cell comprising two polarizable microelectrodes and the influence of impurities on the cell response. Journal of electroanalytical chemistry 2013, 688, 354-359.

31. Wipf, D.O.; Bard, A.J., Scanning Electrochemical Microscopy .7. Effect of Heterogeneous Electron-Transfer Rate at the Substrate on the Tip Feedback Current. J Electrochem Soc 1991, 138, 469-474.

32. Macpherson, J.V.; Slevin, C.J.; Unwin, P.R., Probing the oxidative etching kinetics of metals with the feedback mode of the scanning electrochemical microscope. J Chem Soc Faraday T 1996, 92, 3799-3805.

33. Selzer, Y.; Turyan, I.; Mandler, D., Studying heterogeneous catalysis by the scanning electrochemical microscope (SECM): The reduction of protons by methyl viologen catalyzed by a platinum surface. J Phys Chem B 1999, 103, 15091517.

34. Zoski, C.G.; Simjee, N.; Guenat, O.; Koudelka-Hep, M., Addressable microelectrode arrays: Characterization by imaging with scanning electrochemical microscopy. Anal Chem 2004, 76, 62-72.

35. Xiong, H.; Guo, J.D.; Amemiya, S., Probing heterogeneous electron transfer at an unbiased conductor by scanning electrochemical microscopy in the feedback mode. Anal Chem 2007, 79, 2735-2744.

36. Oleinick, A.I.; Battistel, D.; Daniele, S.; Svir, I.; Amatore, C., Simple and Clear Evidence for Positive Feedback Limitation by Bipolar Behavior during Scanning Electrochemical Microscopy of Unbiased Conductors. Anal Chem 2011, 83, 48874893. 


\section{Potentiometry at nanogaps for ultra-low concentration detection}

We present an electrochemical analysis system that can be employed for the detection of redox species down to sub-nanomolar concentrations. Our system consists of a nanogap cell with two polarizable electrodes, only one of which is under potential control. The concentration of reversible redox species in the sample solution can be detected by measuring the electrostatic potential of the freely floating electrode, which acts as a charge accumulator. The resulting potentiometric signal from the floating electrode is readily measured as a function of the concentration of redox species and provides an alternative method to detect trace quantities of analyte molecules. 


\subsection{Introduction}

Electrochemical sensors permit detection and monitoring of a plethora of analytes over a wide range of concentrations. The detection of trace amounts of redox species is being avidly pursued with applications ranging from basic research to medical diagnostics. ${ }^{1-12}$ Prominent examples include detection of neurotransmitters in the brain, ${ }^{13-18}$ monitoring secretion of electroactive enzymes ${ }^{19-21}$ and the detection of macromolecular species such as DNA. ${ }^{22-26}$ With the advent of miniaturization techniques, high surface-to-volume ratios can be achieved which has led to the development of highly sensitive detection methods. ${ }^{1-5}$ Integration of sensing devices together with the associated readout circuitry on a monolithic chip-based platform is also gaining momentum. ${ }^{27-33}$ Several electrochemical sensing techniques (amperometric, potentiometric, conductometric, etc.) are available, ${ }^{1,3}$ any of which can in principle be coupled with state-of-the-art microelectronic technology for highly scalable integrated sensing platforms. Although successfully implemented and extensively used in scaling down of conventional sensing techniques, further reduction of sensor dimensions also raises the possibility of exploring alternate detection mechanisms. With this in mind, here we propose an electrochemical detection mechanism based on potentiometry that, we believe, could improve detection thresholds, especially at ultra-low concentration limits of detection. Not only can this approach be readily integrated with conventional chip-based readout electronics, but the readout mechanism is also simplified.

In 1962, Delahay proposed the coulostatic method for trace analysis. ${ }^{34}$ In brief, a fixed amount of charge or a current impulse from a charged capacitor of a known value was injected to an electrode. He proposed that the injected charge would be used up by electrochemical reaction and this would result in a subsequent drop in the potential of the electrode. Thus, the decay in the potential could be recorded for electrochemical analysis and the rate of decay was dependent on the concentration of the analyte. Due to the large surface areas of the electrodes that led to high electrical double-layer capacitances, this technique could not be used for ultra-low limits of detection (LOD). Today, however, owing to the advancement in fabrication techniques, electrochemical sensors can be shrunk down to micrometer and even nanometer scales. ${ }^{14,35-39}$ Moreover, if warranted it has become possible to fabricate multiple electrodes separated by distances as low as a few tens of nanometers. ${ }^{40}$ Conventional amperometric detection schemes have already utilized such nano- 
(a)

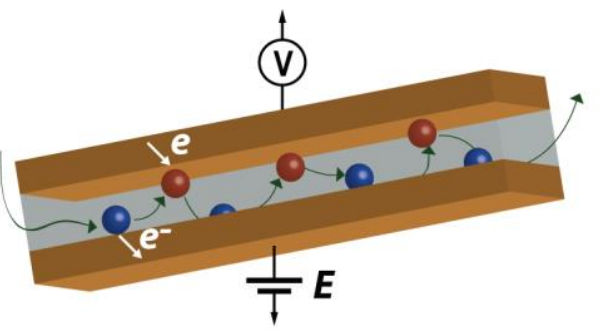

(b)
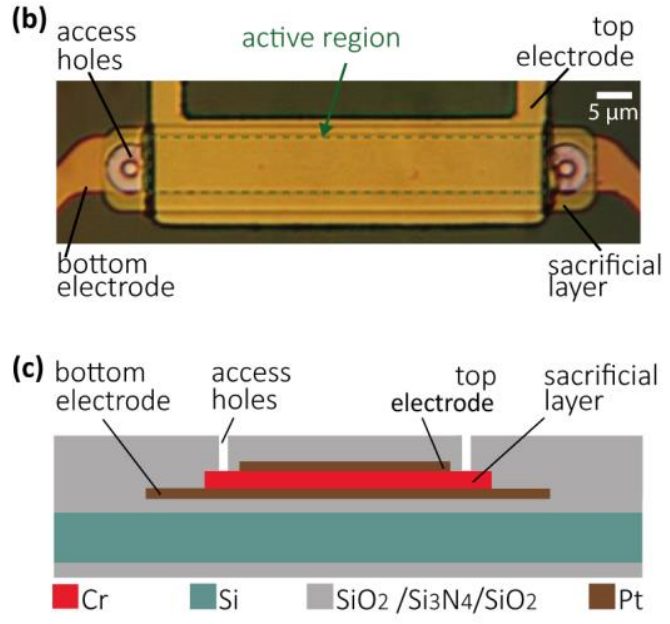

Figure 4.1. a) Schematic showing a nanogap consisting of two parallel polarizable electrodes that are a few tens of nanometers apart. The top electrode is freely floating while a potential is applied to the bottom electrode. A reversible redox molecule shuttling between the two electrodes within the confined space of the nanogap undergoes redox cycling thereby charging up the floating electrode. The change in the potential of the floating electrode is used as the signal readout. b) Optical microscopy image of the top view of a nanogap device consisting of a chromium layer sandwiched between two platinum electrodes. Once sacrificial chromium layer is etched away, the overlapping region between the electrodes serves as the nanogap. c) Schematic diagram of the cross section of the nanogap device prior to etching (not drawn to scale). The height of the nanogap is determined by the thickness of the chromium layer.

spaced devices for the detection of electroactive species down to concentrations approaching or surpassing the single-molecule limit. ${ }^{1,3}$ 35-37 The basic approach, known as redox cycling, is illustrated in Figure 4.1a. Bard and coworkers claimed 
single molecule detection of redox species using scanning electrode microscopy (SECM) by confining molecules between a substrate and a SECM tip. ${ }^{35-37}$ Our group has presented a variant of this concept by fabricating reproducible solid-state devices to create so-called nanogap electrodes for the detection of electroactive species. ${ }^{39-}$ ${ }^{41}$ While these sensors are highly efficient and reliable, ultra-low concentration measurements, being highly susceptible to noise and interference, ${ }^{40}$ still require extensive signal filtering and/or conditioning. While often acceptable in a laboratory setting, this complicates a chip-based implementation where a better optimized detection mechanism would be desirable. With this in mind, here we explore an alternate detection method and evaluate its potential for simplifying read-out mechanisms.

Our potentiometric principle of detection is sketched in Figure 4.1a. At its core is a solid-state device (Figure 4.1b and c) consisting of two polarizable electrodes, one of which is at a known and controlled potential while the other is freely floating (unbiased). Electrochemical reactions occur at the biased electrode in the presence of electroactive analyte molecules. The rapid Brownian shuttling of molecules between the electrodes causes charging of the double layer capacitance of the floating electrode, and the rate at which this occurs is dependent in the concentration of analyte molecules in the solution.

In comparison to more conventional amperometric techniques of detection, this type of potentiometry within nanogaps has two main advantages. First, the output signals are in terms of potential, which simplifies readout. This is particularly relevant for integration with solid-state electronics. Second, signals of up to several $\mathrm{mV}$ can be obtained even at very low concentration on the order of tens of pM.

Zhu et al. have also demonstrated a modified version of Delahey's coulostatic method in a sensor consisting of interdigitated electrodes (IDE). ${ }^{30,42}$ Instead of using a conventional capacitor, they demonstrated the discharge of the double layer capacitance of a microelectrode. A significant decay rate could be measured and concentrations down to a few nM were detected, pointing the way for the present work. Pure potentiometric techniques based on macroscopic electrodes and ionspecific partitioning in solid-state membranes have also achieved supreme levels of sensitivity; ${ }^{43-49}$ to what extent the nanoelectrode approaches introduced here can be coupled to those well-established macroscopic techniques is an open and interesting question. 


\subsection{Theory}

Nanogap sensors consist of two electrodes that are separated by a few tens of nanometers. Here only one of the electrodes is biased or driven to a known potential (driving electrode), leaving the other electrode unbiased (floating electrode). Based on the potential applied to the driving electrode, the species present within the channel may undergo oxidation or reduction. For example, Figure $4.2 a$ shows a situation wherein the driving electrode is held at an oxidizing potential. A molecule that is in its reduced form, upon encountering the driving electrode, gives up an electron and gets oxidized. The molecule thereafter diffuses to the floating electrode which is in close proximity. The floating electrode is capable of reducing the molecule, thereby losing an electron and charging up its own double-layer capacitance, $C_{f}$. As
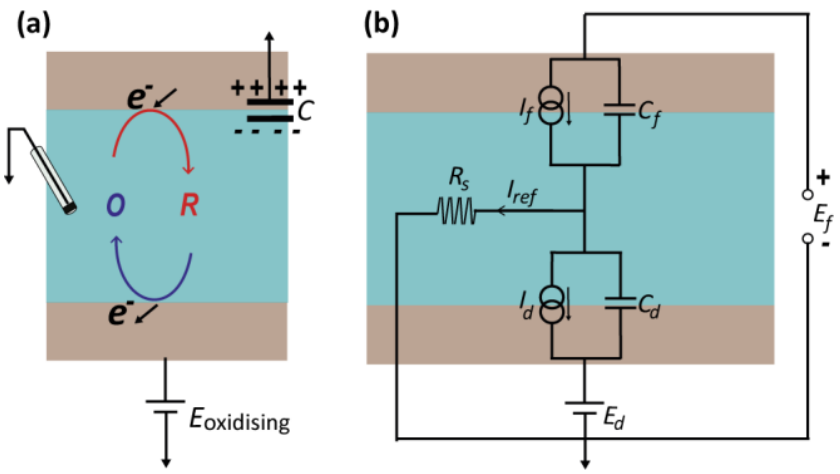

Figure 4.2. a) Schematic showing potentiometric detection mechanism. A reversible redox molecule shuttling between an unbiased floating and biased electrode. Double layer capacitance at the floating electrode, $C_{f}$, charges up due to the shuttling of the molecules which leads to the development of a potential across the interface. The floating electrode accumulates charge over time as the molecule undergoes redox cycling, being successively reduced (shown in red) and oxidized again (shown in blue). b) Equivalent circuit of the potentiometric detection scheme in nanogaps. $C_{f}$ and $C_{d}$ are the double layer capacitances at the floating and driving electrodes, respectively; current through the driving and floating electrodes ( $I_{f}$ and $l_{d}$, respectively) are nearly equal in magnitude current while a current $I_{\text {ref }}$ flows through the reference electrode to complete the circuit. $E_{f}$ measured at the floating electrode is the measured readout signal.

the redox molecule further shuttles back and forth between the two electrodes, it undergoes repeated oxidation and reduction and $C_{f}$ gets increasingly charged. This 
accumulation of charge gradually results in a change in potential of the floating electrode. Any potential difference between the two electrodes influences further redox cycling, until the potential of the floating electrode approaches that of the driving electrode. At this point both electrodes have similar potentials and the process stops. Figure $4.2 \mathrm{~b}$ shows the equivalent circuit diagram for this scenario. When an oxidizing potential is applied to the driving electrode, currents, $l_{d}$ and $l_{f}$, flow through the driving and floating electrodes. While nearly equal in magnitude, in principle a residual current / ref also flows to the reference and completes the circuit.

In order to demonstrate this principle, we apply a time-varying (triangular) potential to the driving electrode, $E_{d}$, and monitor how the floating electrode, $E_{f}$, follows (or tracks) the driving potential as a function of analyte concentration. Initially the floating electrode is at its rest potential. Any difference in potential between the two electrodes results in redox cycling which, as reported previously and employed in Chapter 3, is well described by Butler-Volmer kinetics. ${ }^{41}$ As $E_{\mathrm{d}}$ is varied, rapid shuttling of the molecules result in redox cycling and the double layer capacitance at the floating electrode $C_{d}$ begins to charge up. Thus, over time, the floating electrode behaves like a charge integrator and its potential, $E_{f}$, varies with time as

$$
E_{f}(t)=\frac{1}{C_{f}} \int I(t) d t
$$

The current due to redox cycling within a thin layer cell can be described $a s^{41}$

$$
I_{F}=\frac{i_{\lim }\left(\frac{1}{1+e^{-f \eta_{f}}}-\frac{1}{1+e^{-f \eta_{d}}}\right)}{1+\frac{D}{k_{0} z}\left(\frac{e^{\alpha f \eta_{f}}}{1+e^{f \eta_{f}}}+\frac{e^{\alpha f \eta_{d}}}{1+e^{f \eta_{d}}}\right)} .
$$

Here $z$ is the height of the nanochannel, $f=F / R T$ with $F$ being the Faraday constant, $R$ the gas constant and $T$ the absolute temperature. $\eta_{d}$ is the overpotential applied to the driving electrode, $E_{d, f}-E_{o}^{\prime}$ where $E_{o}^{\prime}$ is the formal potential, $D$ is the diffusion coefficient of the redox species, $k_{0}$ is the heterogeneous rate constant, $\alpha$ is the transfer coefficient, and $i_{\lim }=\frac{n F A D c}{z}$ is the limiting current which is directly proportional to the concentration of the analyte molecules, $c$, and the overlapping area of the two electrodes, $A$. In principle, the floating electrode always tends to track the driving electrode if there exists any difference between the potentials of the two electrodes. However, the rate of tracking is maximum at potentials near $E_{0}^{\prime}$ and 
significantly decreases when both electrodes are either a few $k T(\sim 50 \mathrm{mV})$ above or below the formal potential.

\subsection{Materials and methods}

Device fabrication. Nanogap sensors, as shown in Figure 4.1b and Figure 4.1c, were used in our experiments. The method for the micro-fabrication of the devices was similar to that reported previously. ${ }^{38}$ In brief, the device consisted of a sacrificial chromium layer deposited between two platinum electrodes. The areas of the top and bottom electrodes were $300 \mu^{2}$ and $170 \mu \mathrm{m}^{2}$, respectively, whereas the overlapping area between the two electrodes where redox cycling took place was $150 \mu \mathrm{m}^{2}$. Nanogaps of two different gap sizes, Type I and II, with $z=60 \pm 5 \mathrm{~nm}$ and $40 \pm 5 \mathrm{~nm}$, respectively, were used.

Reagents. Prior to the measurements, a chromium etchant (BASF, Chromium Etch Selectipur) was purged through the inlets in order to etch the sacrificial layer and create the nanochannel. The electrodes were cleaned before conducting the measurements by cycling their applied potential in $5 \mathrm{mM} \mathrm{H}_{2} \mathrm{SO}_{4}$. All solutions were prepared in $18 \mathrm{M} \Omega \mathrm{cm}$ Milli-Q water (Millipore). Ferrocenedimethanol $\left(\mathrm{Fc}(\mathrm{MeOH})_{2}\right.$, Sigma-Aldrich, cat. no- 372625, diffusion coefficient $D=6.7 \times 10^{-10} \mathrm{~m}^{2} \mathrm{~s}^{-1}$ ) was chosen as a simple prototypical redox species and was prepared at different concentrations with $0.1 \mathrm{M} \mathrm{KCl}$ (Sigma-Aldrich, cat. no-P9333) added as supporting electrolyte.

Measurement setup. A polydimethylsiloxane (PDMS) reservoir filled with analyte solution was positioned above the device and a standard $\mathrm{Ag} / \mathrm{AgCl}$ electrode (BASi, MF 2079, RE-5B) inserted in this reservoir was used as the reference electrode. No auxiliary electrode was necessary as the current through the reference electrode remained well below $1 \mathrm{nA}$. In order to ensure a constant input impedance during potentiometric measurements and thus avoid instrumental artefacts, we monitored the floating electrode potential by connecting this electrode via a high impedance resistance of known value $\left(R_{v}=500 \mathrm{G} \Omega\right)$ and monitoring the resulting current, i, through this resistor. The measured potential, $E_{f}$, was calculated by using Ohm's law $\left(E_{f}=R_{v} i_{i}\right)$. The current flowing through the unbiased electrode was observed using a transimpedance amplifier (Femto, DDPCA-300). The main advantage of this approach was that the input impedance of the circuit measurement could be verified to prevent contamination from leakage currents. Further details are presented in the Appendix B. 


\subsection{Results and discussion}

We measured the potential of the floating top electrode, $E_{f}$, while the bottom electrode, $E_{d}$, was driven with triangular potential waves (from $0 \mathrm{~V}$ to $0.5 \mathrm{~V}$ and back to $0 \mathrm{~V}$ ), as shown in Figure 4.3a. Figure 4.3b shows the raw data of the potentials obtained at the floating electrode, $E_{f}$, at redox species concentrations ranging from $10 \mathrm{nM}$ to $200 \mu \mathrm{M}$ (violet to red in order of increasing concentration). At any given concentration, $E_{f}$ is initially in equilibrium with the solution at $t=0 \mathrm{~s}$ when $E_{d}$ is biased

(a)

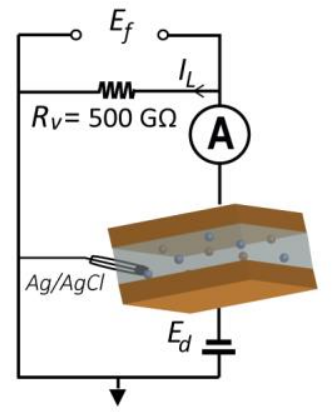

(b)

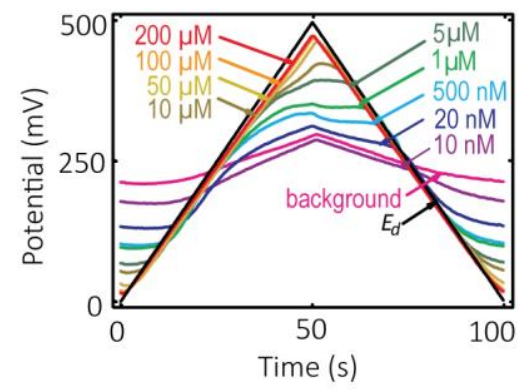

(c)
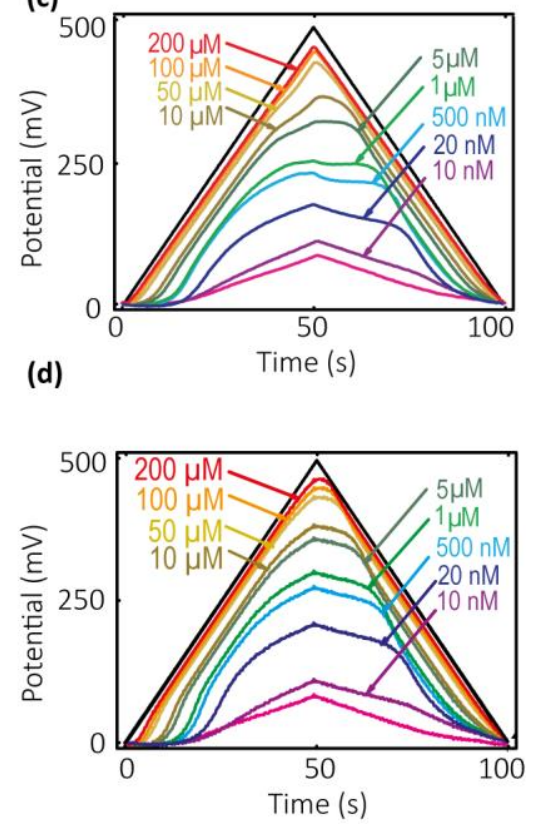

Figure 4.3. a) Experimental setup where the bottom electrode, $E_{d}$, was driven at a range of potentials $(0 \mathrm{~V}-0.5 \mathrm{~V}-0 \mathrm{~V})$ while the top electrode was floating and its potential, $E_{f_{1}}$ was monitored. /, is the leakage current through a $500 \mathrm{G} \Omega$ resistor which was measured for computing the value of $E_{f}$. b) Raw data of measured floating potentials, $E_{f}$, at various concentrations (red to violet, $200 \mu \mathrm{M}$ to $10 \mathrm{nM}$ ) of $\mathrm{Fc}(\mathrm{MeOH})_{2}$ in $0.1 \mathrm{M} \mathrm{KCl}$ solution as background electrolyte. The pink curve shows the potential of the floating electrode in the absence of redox molecules and is referred to as the background signal. c) Same data as in b) but with the starting potential $\left(E_{f}(t=0 \mathrm{~s})=E_{f}(t=100 \mathrm{~s})\right)$ subtracted. $\left.\mathrm{d}\right)$ Simulation incorporating the empirically determined background signal. $C_{f}=290 \mathrm{pF}$ was used in the simulation. 
at 0 V. For instance, at $200 \mu \mathrm{M}$ concentration (Figure 4.3b, red curve), $E_{f}$ is found to be at $20 \mathrm{mV}$ initially. In the first half of the cycle, as $E_{d}$ is swept towards oxidizing potentials, the difference in potential between the two electrodes drives redox cycling of the species in solution. As discussed earlier, highest cycling efficiencies are obtained when the potentials at the two electrodes are within $\pm 50 \mathrm{mV}$ of the formal potential ( $E_{0}^{\prime}=250 \mathrm{mV}$ in our case). Thus, tracking continues efficiently until the potential of the floating electrode becomes so high that both electrodes become oxidizing and the process of redox cycling slows down. In the second half of the cycle, the same process repeats in reverse, i.e. the driving electrode approaches the reducing potential and decreases gradually to $0 \mathrm{~V}$. This results in a tracking of the potential in the reverse direction and both electrodes eventually reach a reducing potential, at which point redox cycling slows down again. $E_{f}$ finally returns to its initial potential (at $E_{d}=0 \mathrm{~V}$ ). When this process is repeated at several different analyte concentrations, we observe that the rate of tracking significantly increases with increasing concentration. For clarity, Figure $4.3 \mathrm{c}$ shows the data of Figure $4.3 \mathrm{~b}$ after an offset potential (starting potential $E_{f}(t=0 \mathrm{~s})=E_{f}(t=100 \mathrm{~s})$ for each separate curve) has been subtracted).

Ideally, in the absence of redox molecules, the floating electrode must not be influenced by any changes in $E_{d}$. However, in practice, trace-level contaminants (reversible and non-reversible species) are unavoidable, causing spontaneous oxidation and/or reduction that also contribute to the charging up of the floating electrode. In Figure $4.3 \mathrm{~b}$ it can be seen that a considerable change in the potential of the floating electrode was observed in the presence of supporting electrolyte only $\left(V_{p p}=88 \mathrm{mV}\right.$, pink curve) even though no redox species were intentionally introduced. We refer to this response as the background signal, Ebg. Several contaminants apart from the analyte of interest are present in the sample (oxygen, organic contamination from air, contamination from earlier reagents like chromium etchant, etc.) which undergo oxidation or reduction and thereby give rise to a parasitic background signal. This signal is reproducible for a given solution, which allows subtracting it to obtain a response due only to the target analyte. We also integrated this background contribution in the theoretical model by rewriting Equation 4.1 as

$$
E_{f}(t)=\frac{1}{c_{d}} \int I_{r c}(t) d t+\frac{1}{c_{d}} \int I_{b g}(t) d t
$$

In practice, the current at the floating electrode due to the background, $I_{b g}$, was backcalculated from the background potential measurement. Simulations were done on 
LABVIEW and the resulting potential at the various concentrations is shown in Figure $4.3 \mathrm{~d}$. The simulated data reproduce the main features of the experimental data, indicating that the model represents a good representation of the functioning of the device.

For the purpose of calculations, the double-layer capacitance of the floating electrode can be estimated since both the area of the electrode and the concentration of the electrolyte are known. ${ }^{50}$ In practice, however, two additional capacitances also accumulate charge due to faradaic processes at the floating electrode. Firstly, the insulation layer $\left(\mathrm{SiO}_{2} / \mathrm{SiN} / \mathrm{SiO}_{2}\right)$ used for passivation of the contacting wires contributes to the capacitance. This is quantitatively negligible, however. Secondly, the connecting cables used within the experimental system to connect the floating electrode to the measurement electronics must be accounted for. The final value of the capacitance of the floating electrode is estimated to be 290 pF, as described in detail in Appendix B.

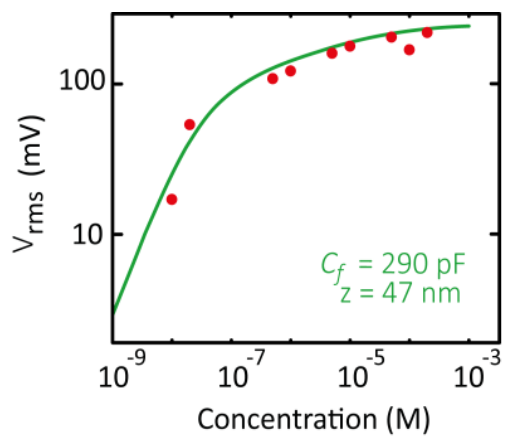

Figure 4.4. Root-mean-square voltage response, $V_{\text {rms, }}$ of the measured potential as a function of the concentration of redox molecules (red dots). This was done after the subtraction of the background signal from $E_{f}$. The solid yellow curve shows $V_{\text {rms }}$ as calculated from the analytical model.

To quantify the degree of tracking of the floating electrode, we computed the rootmean-square value of the measured potential, $V_{\text {rms. }}$. The results as a function of the concentration of redox molecules is shown in Figure 4.4. For comparison, the simulated results based on Equation 4.4 are also presented. In both cases the background signal was first subtracted and thereafter $V_{\text {rms }}$ was computed. The rate at which the floating electrode charges up is dependent on the concentration of redox species within the nanogap. At low concentrations, $V_{\text {rms }}$ changes linearly with increasing concentration. However, at high concentrations of redox molecules $(>100$ 
$\mu \mathrm{M})$, the floating electrode starts to track the applied potential rather closely. In other words, $E_{f}$ tracks $E_{d}$ nearly instantaneously and any further increase in the concentrations does not significantly change the rate of tracking. This behavior fits quite well with the values obtained from the simulated model, as also shown in Figure 4.4 (solid yellow line).

Having understood the relatively simple cases in the high concentration regime, we now turn to lower concentrations. To render this possible, two important changes were made to the experiments. Firstly, Type 2 devices were employed; owing to the smaller gap size $(z \sim 47 \mathrm{~nm})$ the cycling efficiencies were significantly enhanced. The background was also heavily suppressed by the use of a high purity $\mathrm{KCl}$ salt (Sigma Aldrich, cat. No- P451029) Finally, the background signal was checked before

(a)

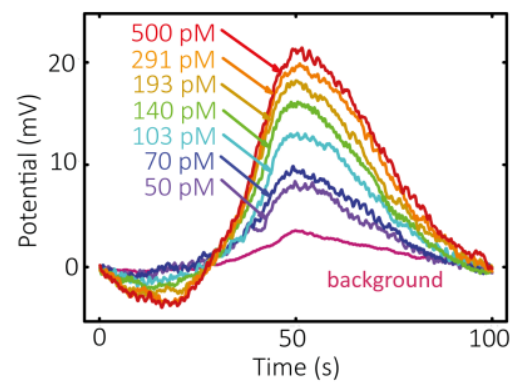

(b)

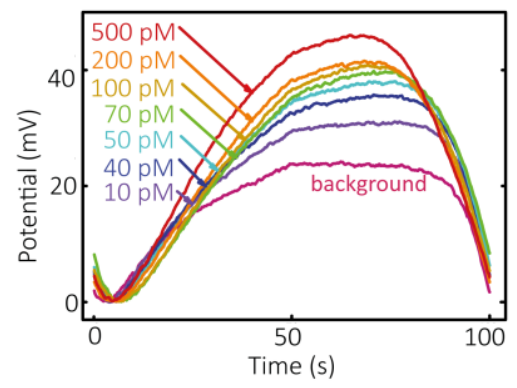

(c)

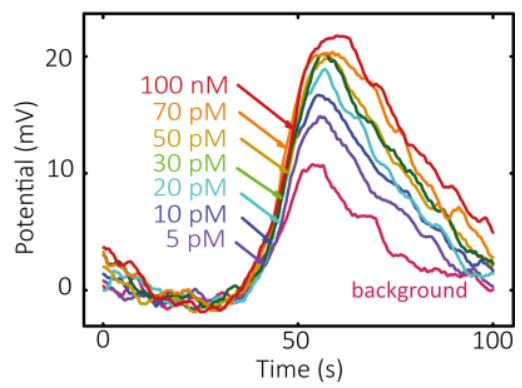

(d)

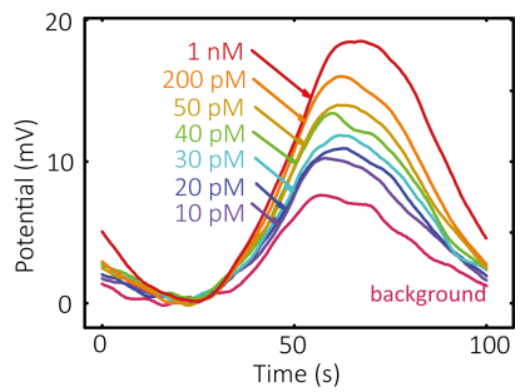

Figure 4.5. Potential of the floating electrode, $E_{f}$, at low concentrations (from $5 \mathrm{pM}$ to $1 \mathrm{nM} \mathrm{Fe}(\mathrm{MeOH})_{2}$ in $\left.0.1 \mathrm{M} \mathrm{KCl}\right)$. These measurements were done in Type 2 devices. The bottom electrode ( $E_{\mathrm{d}}$, not shown) was driven $(0 \mathrm{~V}-0.5 \mathrm{~V}-0 \mathrm{~V}$ ) while the top electrode potential, $E_{f}$, was measured. The pink curve shows the background which was measured in the supporting electrolyte of high purity. The curves are offset corrected for clarity. Panels (a)-(d) show the results obtained on four similar devices in separate measurements. 
beginning the measurement to ensure that it was smaller than the expected signal from the analyte. For example, in Figure 4.5a, when $E_{d}$ was driven between $0 \mathrm{~V}$ to 0.5 $V$ in the supporting electrolyte only, a background signal was observed which was stable over time and was reasonably low ( $V_{p p}=3.5 \mathrm{mV}$, pink curve). Thereafter redox active molecules were introduced into the nanogap. The concentration of the redox species in the solution was increased in steps and the corresponding floating potential sweeps were measured. Measured traces for four different devices are shown in Figure 4.5. In each case the potential at the lowest concentration (5 to 50 pM) is clearly distinguishable when compared to the background signal. As the concentration of redox species was increased, the response of the potential at the floating electrode gradually grew due to redox cycling.

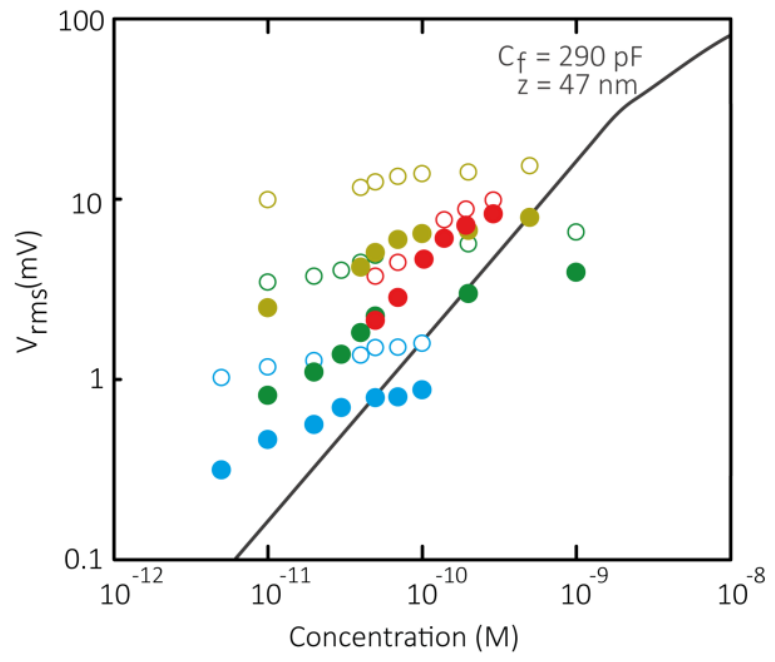

Figure 4.6. Root-mean-square voltage response, $V_{\text {rms, }}$, from the background-subtracted measured potential response at low concentrations ( $5 \mathrm{pM}$ to $1 \mathrm{nM}$ ). Red, blue, green and yellow colors correspond to the measurements of Figure 4.5 a, b, c and d, respectively. The empty and solid circles represent $V_{\text {rms }}$ computed before and after background subtraction, respectively. Simulations (shown as grey line) were performed using a capacitance of the floating electrode of $C_{f}=290 \mathrm{pF}$.

The relationship between $V_{\text {rms }}$ and the concentration is plotted in Figure 4.6 along with the simulated results (grey line). Red, yellow, blue and green colors represent the obtained results from the data shown in Figure 4.5 a,b,c and d, respectively. The change in the potential of the floating electrode ncreases monotonically with 
increasing analyte concentration. This trend is visible at concentrations as low as 5 $\mathrm{pM}$, which corresponds to an average number of molecules in the device $\langle N\rangle=0.03$. Although $V_{\text {rms }}$ increases with increasing concentration, a linear dependence can however not be observed. We believe that at such ultra-low concentrations the background signal interferes with the analyte signal and therefore that the simple background subtraction procedure is no longer accurate. Thus, while each measurement exhibits a monotonic increase in signal with increasing concentrations, the absolute concentration cannot be determined. This implies that, while measuring ultra-low concentrations is possible, trace level contaminants compete with the analyte; accounting for this quantitatively would require a priori accurate knowledge of the contaminant composition.

\subsection{Conclusion and outlook}

We have introduced a redox-cycling based potentiometric method to detect ultralow concentrations. At relatively high concentrations we achieve good agreement with theory, while at ultra-low concentrations (below the single-molecule limit) there is a monotonic increase in signal with increasing concentration, allowing us to distinguish between minor differences in analyte concentration.

Here we employed a triangular-wave applied potential to best demonstrate the working principle. However, for real-time applications, the signal obtained from the response to a step in the driving electrode potential would be more practical. The signal levels reported here are modest compared to what is ultimately possible by reducing the capacitance of the floating electrode by a few orders of magnitude. For example, for a nanometer sized disc electrode (radius of $100 \mathrm{~nm}$ ), the double layer capacitance would be of order $10 \mathrm{fF}$; even one fA of redox-cycling current could then generate a response in the order of $100 \mathrm{mV}$ in one second. Achieving this extreme limit would however require integration of the readout electronics on the same chip as the nanofluidic device to minimize the capacitance of the wires to the electrode.

\subsection{References}

1. Oja, S.M.; Fan, Y.S.; Armstrong, C.M.; Defnet, P.; Zhang, B., Nanoscale Electrochemistry Revisited. Anal Chem 2016, 88, 414-430.

2. Mathwig, K.; Aartsma, T.J.; Canters, G.W.; Lemay, S.G., Nanoscale Methods for Single-Molecule Electrochemistry. Annu Rev Anal Chem 2014, 7, 383-404. 
3. Oja, S.M.; Wood, M.; Zhang, B., Nanoscale Electrochemistry. Analytical Chemistry 2013, 85, 473-486.

4. Mao, B.W., Special issue - Electrochemistry at nanoscale - Structuring, characterization and theories - Preface. Electrochim Acta 2005, 50, 2987-2987.

5. Schuster, R.; Kirchner, V.; Xia, X.H.; Bittner, A.M.; Ertl, G., Nanoscale electrochemistry. Phys Rev Lett 1998, 80, 5599-5602.

6. Wang, Y.; Xu, H.; Zhang, J.M.; Li, G., Electrochemical sensors for clinic analysis. Sensors-Basel 2008, 8, 2043-2081.

7. Yogeswaran, U.; Chen, S.M., A review on the electrochemical sensors and biosensors composed of nanowires as sensing material. Sensors-Basel 2008, 8, 290-313.

8. Bandodkar, A.J.; Wang, J., Non-invasive wearable electrochemical sensors: a review. Trends Biotechnol 2014, 32, 363-371.

9. Stradiotto, N.R.; Yamanaka, H.; Zanoni, M.V.B., Electrochemical sensors: A powerful tool in analytical chemistry. J Brazil Chem Soc 2003, 14, 159-173.

10. Pohanka, M.; Skladai, P., Electrochemical biosensors - principles and applications. J Appl Biomed 2008, 6, 57-64.

11. Aizawa, M., Principles and Applications of Electrochemical and Optical Biosensors. Anal Chim Acta 1991, 250, 249-256.

12. Wang, J., et al., DNA electrochemical biosensors for environmental monitoring. A review. Anal Chim Acta 1997, 347, 1-8.

13. Alvarez-Martos, I.; Ferapontova, E.E., Electrochemical Label-Free Aptasensor for Specific Analysis of Dopamine in Serum in the Presence of Structurally Related Neurotransmitters. Anal Chem 2016, 88, 3608-3616.

14. Bucher, E.S.; Wightman, R.M., Electrochemical Analysis of Neurotransmitters. Annual Review of Analytical Chemistry, Vol 8 2015, 8, 239-261.

15. Parrot, S.; Neuzeret, P.C.; Denoroy, L., A rapid and sensitive method for the analysis of brain monoamine neurotransmitters using ultra-fast liquid chromatography coupled to electrochemical detection. J Chromatogr B 2011, 879, 3871-3878.

16. Zeng, Y.; Chen, H.; Pang, D.W.; Wang, Z.L.; Cheng, J.K., A novel microchip-based capillary electrophoresis system with electrochemical detection and its application to the analysis of neurotransmitters. Chem J Chinese U 2002, 23, 567569.

17. Xia, N.; Liu, L.; Wu, R.J.; Liu, H.P.; Li, S.J.; Hao, Y.Q., Ascorbic acid-triggered electrochemical-chemical-chemical redox cycling for design of enzyme-amplified electrochemical biosensors on self-assembled monolayer-covered gold electrodes. J Electroanal Chem 2014, 731, 78-83.

18. Sajid, M.; Nazal, M.K.; Mansha, M.; Alsharaa, A.; Jillani, S.M.S.; Basheer, C., Chemically modified electrodes for electrochemical detection of dopamine in the presence of uric acid and ascorbic acid: A review. Trac-Trend Anal Chem 2016, 76, 15-29. 
19. Neugebauer, S.; Stoica, L.; Guschin, D.; Schuhmann, W., Redox-amplified biosensors based on selective modification of nanopore electrode structures with enzymes entrapped within electrodeposition paints. Microchim Acta 2008, 163, 33-40.

20. Munro, A.W.; Taylor, P.; Walkinshaw, M.D., Structures of redox enzymes. Curr Opin Biotech 2000, 11, 369-376.

21. Metzner, H., Redox Reactions in Biological-Systems. Electricity and Magnetism in Biology and Medicine 1993, 91-94.

22. Xiao, Y.; Lubin, A.A.; Baker, B.R.; Plaxco, K.W.; Heeger, A.J., Single-step electronic detection of femtomolar DNA by target-induced strand displacement in an electrode-bound duplex. P Natl Acad Sci USA 2006, 103, 16677-16680.

23. Castano-Alvarez, M.; Fernandez-Abedul, M.; Costa-Garcia, A., Electroactive intercalators for DNA analysis on microchip electrophoresis. Electrophoresis 2007, 28, 4679-4689.

24. Kostecka, P.; Havran, L.; Bittova, M.; Pivonkova, H.; Fojta, M., Sensing mispaired thymines in DNA heteroduplexes using an electroactive osmium marker: towards electrochemical SNP probing. Anal Bioanal Chem 2011, 400, 197-204.

25. Nasirizadeh, N.; Zare, H.R.; Pournaghi-Azar, M.H.; Hejazi, M.S., Introduction of hematoxylin as an electroactive label for DNA biosensors and its employment in detection of target DNA sequence and single-base mismatch in human papilloma virus corresponding to oligonucleotide. Biosens Bioelectron 2011, 26, 26382644.

26. Millan, K.M.; Mikkelsen, S.R., Sequence-Selective Biosensor for DNA-Based on Electroactive Hybridization Indicators. Anal Chem 1993, 65, 2317-2323.

27. Ghafar-Zadeh, E., Wireless Integrated Biosensors for Point-of-Care Diagnostic Applications. Sensors-Basel 2015, 15, 3236-3261.

28. Farahi, R.H.; Ferrell, T.L.; Guiseppi-Elie, A.; Hansen, P., Integrated electronics platforms for wireless implantable biosensors. 2007 leee/Nih Life Science Systems and Applications Workshop 2007, 27-30.

29. Du, Y.; Chen, C.G.; Zhou, M.; Dong, S.J.; Wang, E.K., Microfluidic Electrochemical Aptameric Assay Integrated On-Chip: A Potentially Convenient Sensing Platform for the Amplified and Multiplex Analysis of Small Molecules. Anal Chem 2011, 83, 1523-1529.

30. Zhu, X.S.; Ahn, C.H., On-chip electrochemical analysis system using nanoelectrodes and bioelectronic CMOS chip. leee Sensors Journal 2006, 6, 1280-1286.

31. Kruppa, P.; Frey, A.; Kuehne, I.; Schienle, M.; Persike, N.; Kratzmueller, T.; Hartwich, G.; Schmitt-Landsiedel, D., A digital CMOS-based $24 \times 16$ sensor array platform for fully automatic electrochemical DNA detection. Biosens Bioelectron 2010, 26, 1414-1419.

32. Lee, K.H.; Lee, J.O.; Sohn, M.J.; Lee, B.; Choi, S.H.; Kim, S.K.; Yoon, J.B.; Cho, G.H., One-chip electronic detection of DNA hybridization using precision impedancebased CMOS array sensor. Biosens Bioelectron 2010, 26, 1373-1379. 
33. Stagni, C., et al., CMOS DNA sensor array with integratedA/D conversion based on label-free capacitance measurement. leee J Solid-St Circ 2006, 41, 2956-2964.

34. Delahay, P., A New Electroanalytical Method - Coulostatic or Charge-Step Method. Anal Chim Acta 1962, 26, 90-\&.

35. Bard, A.J.; Fan, F.R.F., Electrochemical detection of single molecules. Accounts Chem Res 1996, 29, 572-578.

36. Fan, F.R.F.; Bard, A.J., Electrochemical Detection of Single Molecules. Science $1995,267,871-874$.

37. Fan, F.R.F.; Kwak, J.; Bard, A.J., Single molecule electrochemistry. J Am Chem Soc 1996, 118, 9669-9675.

38. Kang, S.; Mathwig, K.; Lemay, S.G., Response time of nanofluidic electrochemical sensors. Lab on a Chip 2012, 12, 1262-1267.

39. Lemay, S.K.a.S.G., Nanoelectrochemical Methods. In Nanoelectrochemistry, CRC Press: 2015; pp 573-600.

40. Kang, S.; Nieuwenhuis, A.; Mathwig, K.; Mampallil, D.; Lemay, S.G., Electrochemical Single-Molecule Detection in Aqueous Solution Using SelfAligned Nanogap Transducers. Acs Nano 2013, 7, 10931-10937.

41. Zevenbergen, M.A.G.; Wolfrum, B.L.; Goluch, E.D.; Singh, P.S.; Lemay, S.G., Fast Electron-Transfer Kinetics Probed in Nanofluidic Channels. J Am Chem Soc 2009, $131,11471-11477$.

42. Zhu, X.S.; Choi, J.W.; Ahn, C.H., A new dynamic electrochemical transduction mechanism for interdigitated array microelectrodes. Lab Chip 2004, 4, 581-587.

43. Nann, A.; Pretsch, E., Potentiometric Detection of Anions Separated by Capillary Electrophoresis Using an Ion-Selective Microelectrode. J Chromatogr A 1994, 676, 437-442.

44. Bakker, E.; Pretsch, E., Advances in Potentiometry. Electroanal Chem 2012, 24, 1-73.

45. Bakker, E.; Pretsch, E., Nanoscale potentiometry. Trac-Trend Anal Chem 2008, 27, 612-618.

46. Bakker, E.; Pretsch, E., Modern Potentiometry. Angew Chem Int Edit 2007, 46, 5660-5668.

47. Malon, A.; Vigassy, T.; Bakker, E.; Pretsch, E., Potentiometry at trace levels in confined samples: Ion-selective electrodes with subfemtomole detection limits. J Am Chem Soc 2006, 128, 8154-8155.

48. Bakker, E.; Meruva, R.K.; Pretsch, E.; Meyerhoff, M.E., Selectivity of Polymer Membrane-Based Ion-Selective Electrodes - Self-Consistent Model Describing the Potentiometric Response in Mixed Ion Solutions of Different Charge. Anal Chem 1994, 66, 3021-3030.

49. Bobacka, J.; Ivaska, A.; Lewenstam, A., Potentiometric ion sensors. Chem Rev 2008, 108, 329-351.

50. Bard, A.J.; Faulkner, L.R., Electrochemical Methods: Fundamentals and Applications. Wiley: 


\section{Integrated glass microfluidics with electrochemical nanogap sensors}

We present a framework for the fabrication of a chip-based monolithic electrochemical sensor integrated with micro-flow. In the past, polydimethylsiloxane (PDMS) has been used for prototyping of electrochemical nanogap sensors. In the new generation of devices described here, SU- 8 aided adhesive bonding of silicon and glass wafers has instead been used to incorporate fluidic control. The fabrication process meets the requirements of wafer-scale production techniques and has a high throughput and reproducibility. Additionally, the devices are packaged as a platform which allows simple electrical and fluidic connections. In order to demonstrate one of the many utilities of these flow-incorporated nanogap sensors, dopamine hydrochloride, which is well known for fouling electrode surfaces, was measured voltametrically at various flow velocities. It is established that high flow rates within the nanogap provide resistance to fouling of the electrode surfaces, improving the sensitivity of the sensors. 


\subsection{Introduction}

Modern Lab-on-a-chip (LoC) platforms aim at creating plug-and-play systems that easily integrate the various functional elements (electronics, fluidics, sensing) together to create truly sample-in-answer-out systems that are both accurate and portable. ${ }^{1-9}$ Such systems have potential benefits in varied fields of application

(a)

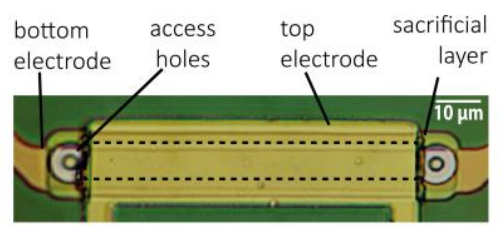

(b)

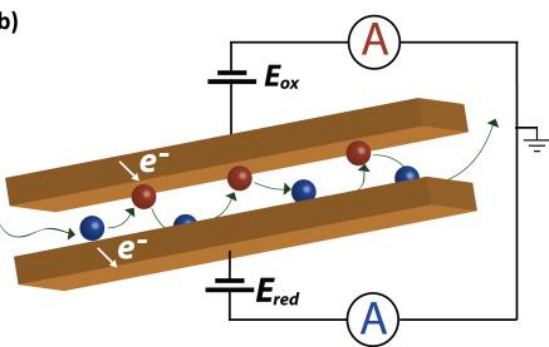

(c)

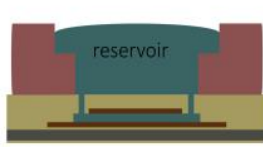

PDMS

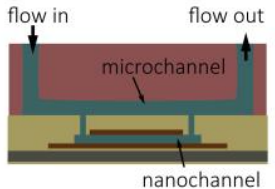

nanochannel

Figure 5.1. a) Optical microscopy image (top view) of a nanogap device consisting of two parallel platinum electrodes sandwiching a chromium layer. This sacrificial $\mathrm{Cr}$ layer is etched to create the nanogap device. b) Schematic diagram of the device showing the top and bottom electrodes biased at oxidizing and reducing potentials, respectively. Molecules entering the nanogap undergo successive oxidation and reduction, thereby giving rise to highly amplified faradaic currents. c) Schematics showing the sample solution in contact with the nanogap device using a PDMS reservoir without flow (left) and with incorporated microfluidics (right). The PDMS reservoir permits the entry of target molecules into the nanogap via diffusion, while the microfluidic channel in a parallel-flow configuration allows for directed advection of the test solution. 
ranging from point-of-care diagnostics to the detection of biohazards. While many prototypical examples have been successfully implemented, ${ }^{10-11}$ there is an everincreasing demand for scaling-down techniques to achieve faster, cheaper and more sensitive systems. ${ }^{4-8}$ In particular it is possible to integrate highly sensitive detection methods with integrated microelectronics for monolithic chip-based platforms. Microfluidics, however, does not follow the same fabrication and scaling rules as electronics and sensing techniques. The field has so far been dominated by soft lithography, 4-5, 8, 12 polydimethylsiloxane (PDMS) being one of the most popular and widely used material. While useful for early prototyping, such methods are difficult to integrate with many other state-of-the-art fabrication techniques. ${ }^{4-6,13}$ There has been a growing interest in fluidic implementations that can be directly realized during wafer-scale fabrication. A commonly used technique is to create fluidic channels directly on a wafer using methods such as anisotropic etching, bulk micromachining, deep ion reactive etching (DRIE) and others, ${ }^{4,14-18}$ and to bond the resulting structures to wafers bearing electronics or sensors in order to create monolithic chipbased fluidic platforms. Such methods are not only favorable for commercialization and large-scale production but also offer advantages such as greater mechanical strength even with high-aspect structures. In the past we have already reported nanogap sensors that count among the most sensitive electrochemical sensors built to date. ${ }^{19}$ Here we report a new generation of devices incorporating wafer-scale integrated glass microfluidics.

\subsection{Microfluidic integration}

Microfabrication. Figure 5.2 shows the process flow for the fabrication of the nanogap sensors incorporated with fluidics. A double-side polished (DSP) 4 inch Si wafer of $525 \mu \mathrm{m}$ thickness was used as starting material. $\mathrm{SiO}_{2}$ of $\sim 500 \mathrm{~nm}$ was thermally grown on both sides. In the first phase of fabrication, the back side of the wafer was processed to initiate the creation of the fluidic inlet and outlet. In step 1, a positive photoresist (OIR 907-12, Arch chemicals) of thickness $1.7 \mu \mathrm{m}$ was deposited and patterned by photolithography and then hard baked $\left(100^{\circ} \mathrm{C}\right)$ for ten minutes. This served as the mask for etching the oxide layer by DRIE using an Adixen SE instrument (Alcatel model AMS SE). Two markers, placed on the wafer edge diametrically opposite to each other, were also etched in the oxide later during this step. In step 2 , the Si was was etched through to a depth of $\sim 475 \mu \mathrm{m}$, the previously patterned silicon oxide effectively acting as mask. A DRIE process was chosen once 


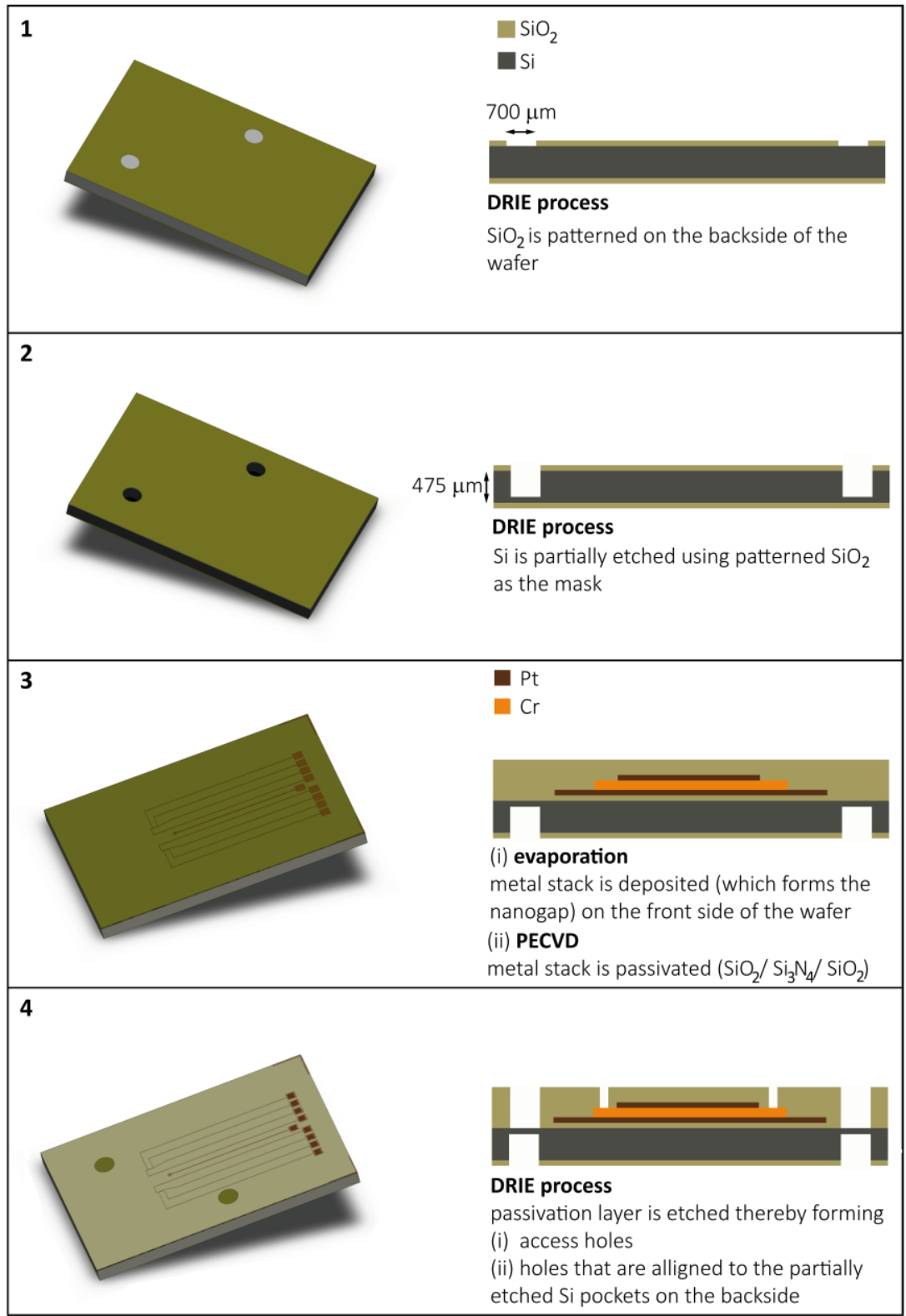

Figure 5.2. Schematic illustration (not drawn to scale) of the different stages of the fabrication process. The left and right columns show three-dimensional sketches and cross- sections of a complete device, respectively. The individual steps are described in detail in the main text.

again due to its directionality such that straight edges could be achieved. This step 


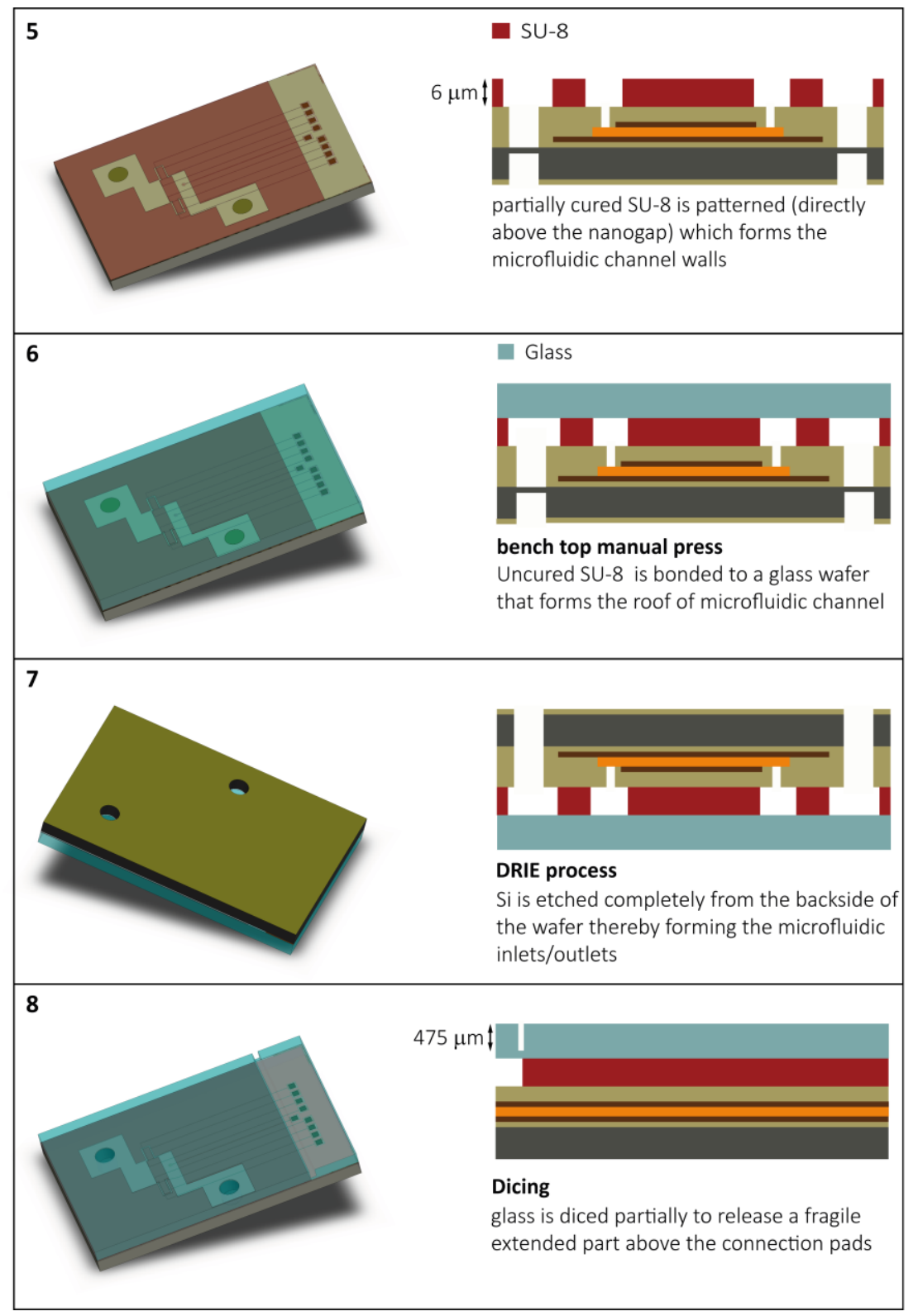

was performed on an Adixen DE instrument (Alcatel model AMS DE). This left $50 \mu \mathrm{m}$ of material intact. The Si wafer was not completely etched through because spin coating of resist on the other side of a wafer bearing holes would be impossible. This completed the first phase of the backside processing of the wafer. Thereafter, in step 
3, the wafer was flipped over and nanogap devices were fabricated on the front side following a previously reported process. ${ }^{21}$ In short a $20 \mathrm{~nm}$ thick Pt bottom electrode, a $60 \mathrm{~nm}$ thick $\mathrm{Cr}$ sacrificial layer and a $120 \mathrm{~nm}$ thick Pt top electrode were sequentially deposited by electron-beam evaporation and patterned using a lift-off process based on a positive photoresist (OIR 907-17, Arch chemicals). The wafer was carefully aligned to the markers on the backside of the wafer in order to ensure the alignment of the micro- and nanofluidic inlet/outlets. Afterwards, a passivation layer consisting of $120 \mathrm{~nm} / 360 \mathrm{~nm} / 120 \mathrm{~nm}$ thick PECVD SiO$/ 2 / \mathrm{SiN}_{\mathrm{SiO}}$ was deposited. In step 4, the passivation layer was patterned and etched in two regions: First, to form the inlet and outlet of the nanogaps by exposing the sacrificial chromium layer. Second, the passivation layer was also etched in the regions bearing the inlet/outlet patterns of the microchannel from the backside. Afterwards (step 5), SU-8 2005 was spin-coated on the top of the nanogap structures. The resulting thickness of the SU-8, $\sim 6 \mu \mathrm{m}$, defined the height of the microchannel. The resist was soft baked by ramping up the temperature to $95^{\circ} \mathrm{C}$ degrees for $30 \mathrm{~min}$ followed by cooling down to room temperature. Following exposure to UV light, the resist was developed; the patterned structures created the walls of the SU-8 micro-channels. The conventional post-bake step was intentionally not performed, which rendered SU-8 only partially cured. Subsequently, this patterned Si wafer was then irreversibly bonded ${ }^{24}$ to a 4 inch Borofloat glass wafer $(525 \mu \mathrm{m})$ at an elevated temperature and pressure $\left(180^{\circ} \mathrm{C}, 1.5\right.$ tons) for $1 \mathrm{hr}$ (step 6). This step was done within a few hours of patterning SU-8 while it was still soft. Finally, in step 7, the leftover silicon was etched away from the backside using the same DRIE process as in step 2 but with a different etcher (SPTS model Pegasus) that allows a stack of wafers (Si/SU-8/glass) to be processed. Additionally, the electrical connection pads needed to be exposed for electrical connections. To achieve this, the SU-8 was patterned in such a way that it did not cover the parts of the wafer that held the electrical contact pads. It was thus possible, in step 8, to cut through the glass wafer partially (down to $475 \mu \mathrm{m}$ ) around the connection pads without disturbing the remaining stack. Finally, the entire wafer was diced into individual chips. The final chip included a fragile glass part extending until the connection pads which was removed with tweezers before using the chip.

We chose this approach over commonly used methods such as anodic bonding because the latter require the application of high temperatures and voltages. This is incompatible with our nanogap sensors since $\mathrm{Cr}$ and $\mathrm{Pt}$ interdiffuse at elevated temperatures. 
(a)

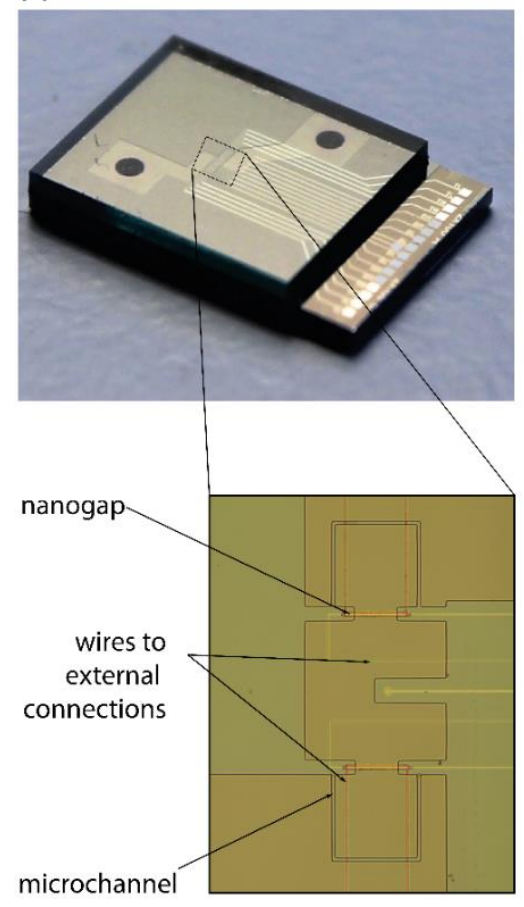

(b)

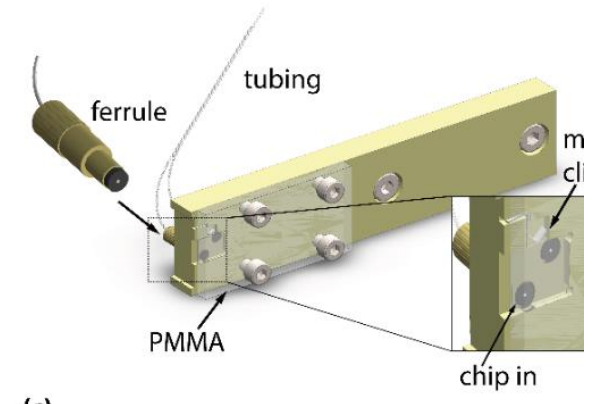

(c)

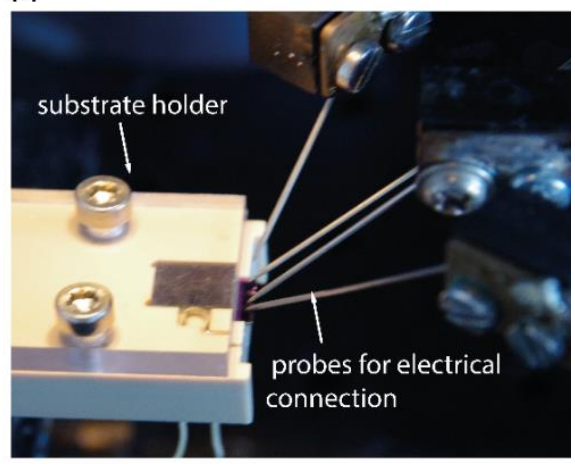

Figure 5.3. a) Photograph of the microfluidic integrated nanogap sensor and zoom in of the device. b) Assembly of the fluidic device for measurements. Fluid is introduced in and out of the chip via the backside of the holder. A metallic clip is required for the tight fitting of the chip in the groove. c) Device held in position during a measurement and connected with needles making contact to the pads on the chip.

Device assembly. The final device integrated with microfluidics is shown in Figure 5.3a. The microfluidic connections were made via the $700 \mu \mathrm{m}$ holes on the backside of the chip. The chip was mounted on a substrate holder as shown in Figure 5.3b. The chip was fitted inside a cavity high enough to accommodate a thickness of ca. 1.05 $\mathrm{mm}$ (Si/ SU-8/glass thicknesses of 525/6/525 $\mu \mathrm{m}$ ). The backside of the chip was pressed against a gasket and ferrule (Idex microfluidics for nanoport assemblies, catalogue no. F-123 $\mathrm{H}$ and $\mathrm{N} 123-03$, respectively) that were screwed into the substrate holder for leak-proof connections. Due to wearing down of the blades during the process of dicing, the lateral dimensions had an error of up to $\sim 50 \mu \mathrm{m}$. For this reason, the diameter of the holes in the chip were about twice the size of the opening of the gasket (360 $\mu \mathrm{m}$ diameter). Thus, any variations between chips could be accommodated for. Finally, a metallic clip was used as a spring to maintain the 


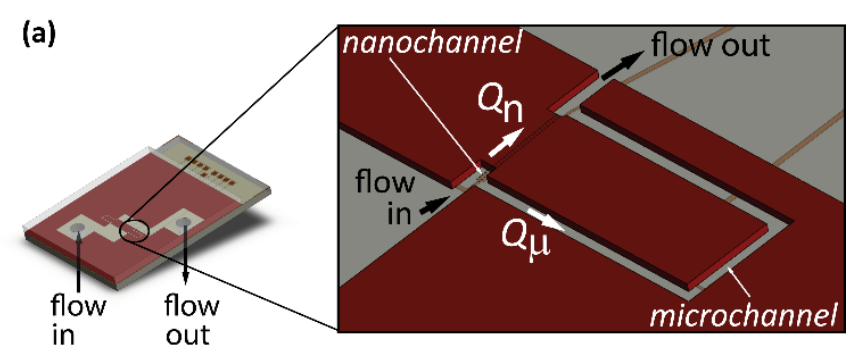

(b)

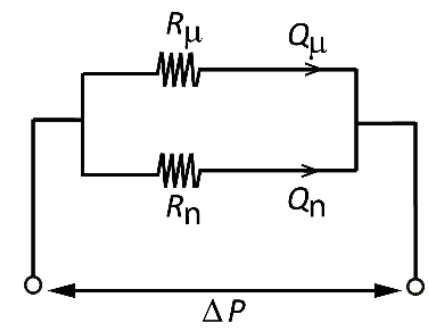

Figure 5.4. a) Schematic of the fluid flow in the chip. b) Equivalent circuit for the fluidic configuration where $R$ is the channel resistances while $Q$ is the flow rate observed at the channel. $\Delta P$ is the pressure difference applied between the inlet and outlet.

position of the chip such that holes in the chip and the holder remained aligned during assembly. A polymethylmethacrylate (PMMA) holder held the chip and fluidic connections in place. Figure 5.3c shows the device held in the substrate during a measurement.

Fluidic connection. We chose a syringe pump (Pump 11 Pico Plus Elite, Harvard Apparatus) to drive the solution and controlled flow rates within the assembled system. Fluid entering the device encounters two parallel channels, the SU-8 microchannel and the nanogap, and is split between these two pathways as shown in Figure 5.4. For a Poiseuille flow in this configuration, the flow rates in the two channels is given by the ratio of their hydraulic resistances, ${ }^{23,25} R$, such that

$$
\frac{Q_{\mu}}{Q_{n}}=\frac{R_{n}}{R_{\mu}} \approx \frac{\left(1-\frac{0.63 h_{\mu}}{w_{\mu}}\right)}{\left(1-\frac{0.63 h_{n}}{w_{n}}\right)} \frac{h_{\mu}^{3} w_{\mu} L_{n}}{h_{n}^{3} w_{n} L_{\mu}}
$$


Here $w, h$, and $L$ represent the width, height and length of the channels, respectively. The dimensions of the nanochannel are defined by the volume initially occupied by the sacrificial $\mathrm{Cr}$ layer. For a nanogap of length $100 \mu \mathrm{m}$ and cross sectional area of 5 $\mu \mathrm{m} \times 65 \mathrm{~nm}$, the resistance has a value $R_{\mathrm{n}} \approx 9 \times 10^{20} \mathrm{~N} \mathrm{~s} / \mathrm{m}$. Thus, for a microchannel, that is $2000 \mu \mathrm{m}$ long and with a cross-sectional area of $35 \mu \mathrm{m}^{2}$, a ratio as high as 18,000:1 was achieved. In practice, two such devices were present on a single chip and four parallel fluidic channels existed. Thus, the flow rate at the nanochannel was reduced by a factor of approximately 36,000 compared to the flow rate provided by an external syringe pump.

\subsection{Experimental results}

Test of wafer adhesion. The system was assembled and put to a leakage test. For this purpose, saturated solution of fluorescein sodium salt (Sigma Aldrich; cat. no- F6377) was passed through the device at an applied inlet pressure of 2.5 bar for about 2 hours and was illuminated using a light of wavelength of $460 \mathrm{~nm}$. Figure $5.5 \mathrm{a}$ shows the resulting optical image of a device at the conclusion of such a test. No fluorescence is observed outside the channel areas, indicating that the SU-8 walls provide a good seal with the top glass wafer.

Voltammetry test. Reversible adsorption of analyte molecules and its irreversible counterpart, electrode fouling, are commonly observed during electrochemical analysis, particularly with biological samples. This affects the overall sensitivity, detection limit, reproducibility and response time of electrochemical sensors. ${ }^{26}$ Fouling mechanisms vary with the nature of the analyte and its interactions with the electrode surfaces and therefore different strategies may be used to prevent or reduce fouling. Adsorption, whether reversible or irreversible, is of particular concern in micro/nanofluidic devices due to their inherently high surface-to-volume.

As a first test of our microfluidic-enabled devices, we performed cyclic voltammetry measurements using ferrocene dimethanol $\left(\mathrm{Fc}(\mathrm{MeOH})_{2}\right.$, Sigma-Aldrich, cat. no372625 , diffusion coefficient $D=6.7 \times 10^{-10} \mathrm{~m}^{2} \mathrm{~s}^{-1}$ ) as redox species as its response is well characterized for nanogap devices. The shape of the voltammogram is known to be influenced by reversible adsorption, the main manifestation being a hysteretic response even at relatively slow scan rates. A nanogap of dimension $100 \mu \mathrm{m} \times 3 \mu \mathrm{m}$ $\times 65 \pm 5 \mathrm{~nm}$ was used for the experiment. Prior to the measurements, chromium etchant (BASF, Chromium Etch Selectipur) was filled through the inlets in order to 
etch the sacrificial layer and create the nanochannel. The electrodes were cleaned before conducting the measurements by cycling their potential in $5 \mathrm{mM} \mathrm{H}_{2} \mathrm{SO}_{4}$. Figure $5.5 \mathrm{~b}$ shows cyclic voltammograms obtained at various flow rates for $1 \mathrm{mM}$ $\mathrm{Fc}(\mathrm{MeOH})_{2}$ in $0.1 \mathrm{M} \mathrm{KCl}$ aqueous solution in a device with a nanochannel length of $100 \mu \mathrm{m}$. As previously reported for such long devices, the voltammogram exhibits

(a)

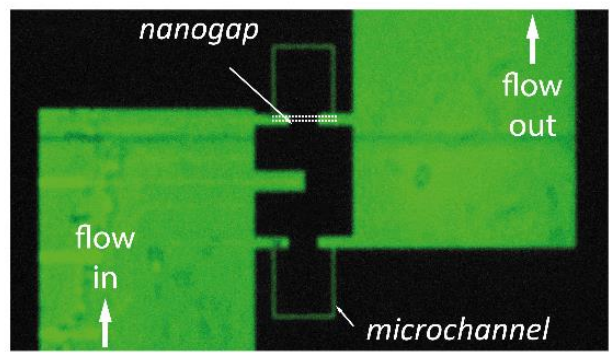

(b)

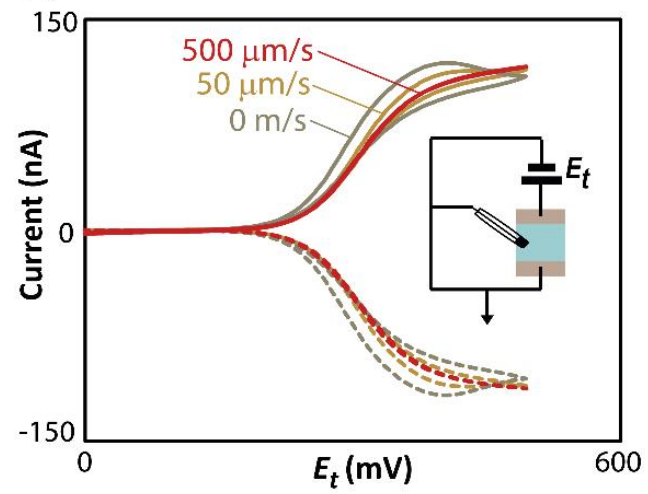

Figure 5.5. a) Optical image of the device at a flow velocity of $500 \mu \mathrm{m} / \mathrm{s}$ to verify the leak-free working of the device. Fluorescent molecules (fluorescein) were used as marker and imaged using a light source with a wavelength of $460 \mathrm{~nm}$. b) Cyclic voltammograms at different flow velocities within the nanogap (sweep rate $10 \mathrm{mV} / \mathrm{s}$ ). The measurements were done using $1 \mathrm{mM} \mathrm{Fc}(\mathrm{MeOH})_{2}$ in $0.1 \mathrm{M} \mathrm{KCl}$ aqueous solution. The solid and dashed lines represent the currents at the top and bottom electrodes, respectively, when the top electrode is swept and the bottom electrode is maintained at $0 \mathrm{~V}$ with respect to $\mathrm{Ag} / \mathrm{AgCl}$. The hysteresis, caused by adsorption of ferrocene on the Pt electrodes, decreases with increasing flow rate and essentially disappears at high flow rates. 
pronounced hysteresis with no flow ${ }^{21}$ or low flow rates. The magnitude of this hysteresis reduces significantly with increasing flow rate, however, and essentially disappears at fluid speeds above $500 \mu \mathrm{m} / \mathrm{s}$, reflecting the fast replenishment of the solution in the nanochannel and the corresponding maintenance of near-steadystate conditions in the nanogap device. This demonstrates explicitly that advective flow can enhance the response of redox-cycling based nanofluidic detectors.

Dopamine. As a more challenging analyte we turn to dopamine, a notable neurotransmitter implicated in brain functions that include pleasure reward, memory and behavior. While several electrochemical methods of detection have been developed, accurate determination of trace amounts of dopamine still represents an experimental challenge. This is due to two main reasons. Firstly, in the brain dopamine is released simultaneously with interfering compounds such as ascorbic acid. These can be found at concentrations a few orders of magnitude higher than that of dopamine and exhibit similar oxidation potentials, making it difficult to separate the dopamine signal from these interferents unless a means is found to provide high selectivity. Secondly, dopamine is a notorious fouling agent, and forms highly reactive intermediates that result in free radical polymerization and ultimately in the formation of melanin. This precipitates unto the electrode surface and creates an insulating layer, inhibiting further surface-dependent redox chemistry ${ }^{26-27}$ unless steps are taken to mitigate this via the choice of electrode material or the introduction of surfactants. Dopamine is a chemically reversible electroactive species, and therefore nanogap sensors provide a high level of signal amplification. It has previously been shown that this can be employed to selectively detect catechol, catechol amines, and dopamine, the former also against a high background of ascorbic acid. ${ }^{28}$ The cyclic voltammograms were, however, characterized by $\mathrm{pH}$ dependent, irreversible ${ }^{28}$ or highly hysteretic, non-standard ${ }^{29}$ shapes. The microscopic origin of these non-standard responses has not been elucidated.

To test whether fluid advection can be employed to mitigate these non-idealities, we recorded cyclic voltammograms at various flow velocities, as shown in Figure 5.6. A schematic of the measurement setup is given in Figure 5.6a. The bottom electrode was left at -200 mV while the top electrode was swept between - $200 \mathrm{mV}$ and 600 $\mathrm{mV}$. A slow scan rate of $10 \mathrm{mV} / \mathrm{s}$ was used to maintain steady-state currents. Dopamine hydrochloride (SigmaAldrich, cat. no- H8502) was prepared with a concentration of $100 \mathrm{nM}$ in $10 \mathrm{mM}$ phosphate buffer to a pH of 7.4 (SigmaAldrich, cat. no-P4417) as supporting electrolyte. Figure 5.6b shows cyclic voltammograms 
without dopamine where the simultaneously measured currents through each of the two working electrodes are plotted. The current at the electrode being cycled (in this case the top electrode, black curve) includes a large capacitive contribution which causes a pronounced hysteresis. This capacitance results in part from the electrical double layer in regions where the electrode is exposed to the solution as well as from the capacitance of the connecting wires, which have a relatively large area and are only protected from the solution by a thin passivating layer of $\mathrm{SiN} / \mathrm{SiO}_{2} / \mathrm{SiN}$. For this reason we concentrate our analysis below on the current measured at the electrode held at the fixed potential (Figure 5.6b, inset, red curve). Although a small ( 33 pA) wave is observed in this case, this is much smaller than the redox cycling current. The

(a)

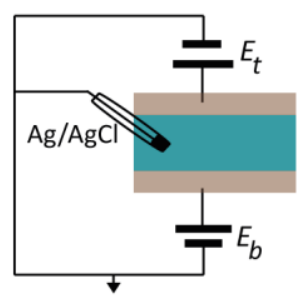

(c)

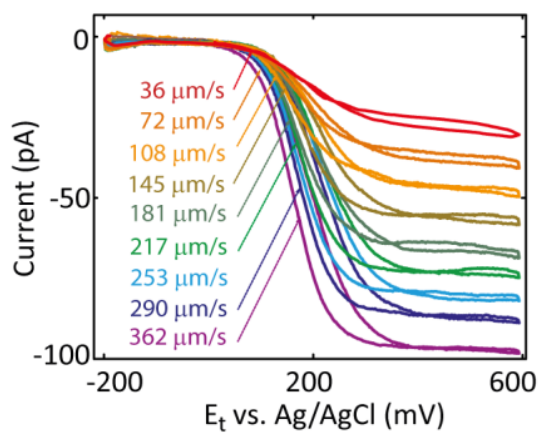

(b)

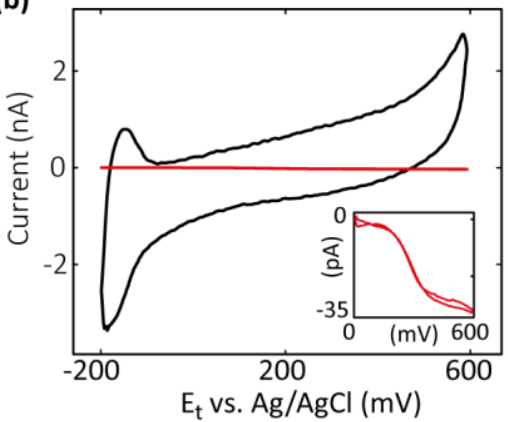

(d)

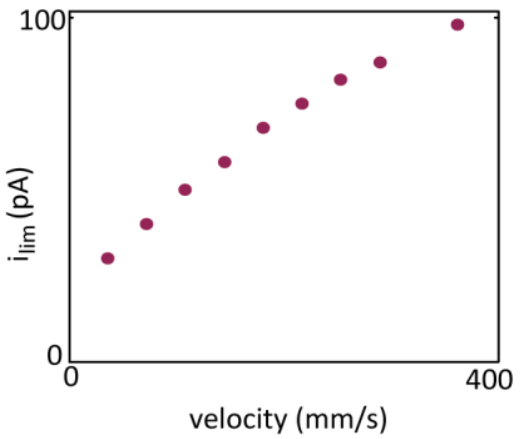

Figure 5.6. a) Experimental configuration in which the potential of the top electrode, $E_{t}$, was scanned between $-0.2 \mathrm{~V}$ and $0.6 \mathrm{~V}$ while the potential of the bottom electrode, $E_{b}$, was maintained at $-0.2 \mathrm{~V}$ (scan rate $10 \mathrm{mV} / \mathrm{s}$ ). b) Current measured at the top (black line) and bottom (red line) electrode in PBS upon scanning $E_{t} . c$ ) Redox cycling current measured at the bottom electrode with $100 \mathrm{nM}$ dopamine in PBS while scanning the top electrode at various flow velocities. d) Limiting current versus flow velocity. 
features observed near $-0.2 \mathrm{~V}$ in both voltammograms instead arise from proton reduction and hydrogen oxidation and are not discussed further.

Figure 5.6c shows the redox cycling current in the presence of $100 \mathrm{nM}$ dopamine for different flow velocities $v$. In each case a sigmoidal curve with a well-defined but slightly hysteretic limiting current $i_{\text {lim }}$ is observed. Here a well-defined wave is observed which we attribute to the dopamine electrochemistry and the half wave potential is observed at $E_{1 / 2}=170 \mathrm{mV}$. Interestingly, the value of $i_{\text {lim }}$ increases monotonically with increasing flow velocity.

The diffusion-limited current for redox cycling in a nanogap geometry is given by $i_{\text {lim }}=n F D A c / z$, where $n$ is the number of electrons transferred, $F$ is the Faraday constant, ${ }^{29} D=6.7 \times 10^{-10} \mathrm{~m}^{2} \mathrm{~s}^{-1}$ and $c=200 \mathrm{nM}$ are the diffusion coefficient and concentration of the redox species, respectively, and $z=65 \pm 5 \mathrm{~nm}$ is the distance between the two electrodes. The value of $i_{\text {lim }}$ is independent of flow rate so long as the residence time for a molecule advecting through the device, $L / v$, is much longer than the transverse diffusion time, $z^{2} / D$; this condition is satisfied until $v$ approaches the experimentally unreachable value of $15 \mathrm{~m} / \mathrm{s}$ and is thus expected to hold for all the results presented here.

The above discussion suggests an expected limiting current $i_{\mathrm{lim}}=120 \mathrm{pA}$ for all fluid velocities investigated. At a low flow speed of $36 \mu \mathrm{m} / \mathrm{s}$, however, only $25 \%$ of the ideal signal could be observed. On the other hand, by increasing the flow rates up to $362 \mu \mathrm{m} / \mathrm{s}$, it was possible to detect $80 \%$ of the signal. The response of dopamine in redox cycling is complex as it depends on surface adsorption and/or fouling, as well as possible local changes in $\mathrm{pH}$ due to proton gradients being created during redox cycling and complex electron transfer kinetics. The present results show that this complexity is at least in part mitigated by rapid advection of the sample through the detection volume, allowing the recovery of near-ideal signal levels.

\subsection{Conclusion and outlook}

We introduced nanogap devices with integrated microfluidic channels which were produced on the wafer scale. Fluidic access from the back side of the wafer combined with electronic access on the front side greatly simplifies interfacing to external instrumentation. This approach further offers a fast method for exchanging analyte solutions and minimizes the risk of contamination. The sensors were successfully tested at pressures of up to 2.5 bar. Cyclic voltammetry experiments were carried 
out with a neurotransmitter well known for fouling electrode surfaces, dopamine hydrochloride. It was shown that an increase in the flow velocities within the nanogap detector leads to a corresponding increase in the limiting current, highlighting the applicability of fluidics-enabled nanogap detectors for the detection and study of complex electroactive species such as dopamine.

\section{$5.5 \quad$ References}

1. Wu, S.Y.; Lin, Q.; Yuen, Y.; Tai, Y.C., MEMS flow sensors for nano-fluidic applications. Sensor Actuat a-Phys 2001, 89, 152-158.

2. Mijatovic, D.; Eijkel, J.C.T.; van den Berg, A., Technologies for nanofluidic systems: top-down vs. bottom-up - a review. Lab Chip 2005, 5, 492-500.

3. Ducree, J.; Glatzel, I.; Brenner, T.; Zengerle, R., Coriolis-induced flow control for micro- and nanofluidic lab-on-a-disk technologies. Micronano Integration 2004, 147-153.

4. Temiz, Y.; Lovchik, R.D.; Kaigala, G.V.; Delamarche, E., Lab-on-a-chip devices: How to close and plug the lab? Microelectron Eng 2015, 132, 156-175.

5. Rios, A.; Zougagh, M.; Avila, M., Miniaturization through lab-on-a-chip: Utopia or reality for routine laboratories? A review. Anal Chim Acta 2012, 740, 1-11.

6. Trietsch, S.J.; Hankemeier, T.; van der Linden, H.J., Lab-on-a-chip technologies for massive parallel data generation in the life sciences: A review. Chemometr Intell Lab 2011, 108, 64-75.

7. Gebauer, A.; Schmidt, S.; Hoffmann, W., Status and perspective of lab-on-a-chip systems for common diseases: a systematic review from 2003 to 2013. Pers Med 2016, 13, 71-91.

8. Abgrall, P.; Gue, A.M., Lab-on-chip technologies: making a microfluidic network and coupling it into a complete microsystem - a review. J Micromech Microeng 2007, 17, R15-R49.

9. van den Berg, A.; Craighead, H.G.; Yang, P.D., From microfluidic applications to nanofluidic phenomena. Chem Soc Rev 2010, 39, 899-900.

10. Gomez, F.A., Paper microfluidics in bioanalysis. Bioanalysis 2014, 6, 2911-2914.

11. Dungchai, W.; Chailapakul, O.; Henry, C.S., Electrochemical Detection for PaperBased Microfluidics. Anal Chem 2009, 81, 5821-5826.

12. Duffy, D.C.; McDonald, J.C.; Schueller, O.J.A.; Whitesides, G.M., Rapid prototyping of microfluidic systems in poly(dimethylsiloxane). Anal Chem 1998, 70, 4974-4984.

13. Iliescu, C.; Taylor, H.; Avram, M.; Miao, J.M.; Franssila, S., A practical guide for the fabrication of microfluidic devices using glass and silicon. Biomicrofluidics 2012, 6 .

14. Wiemer, M.; Jia, C.P.; Toepper, M.; Hauck, K., Wafer bonding with BCB and SU-8 for MEMS packaging. ESTC 2006: 1st Electronics Systemintegration Technology Conference, Vols 1 and 2, Proceedings 2006, 1401-1405. 
15. Hwang, T.J.; Popa, D.; Sin, J.; Stephanou, H.E.; Leonard, E.M., BCB wafer bonding for microfluidics. Micromachining and Microfabrication Process Technology Ix 2004, 5342, 182-191.

16. Hwang, T.J.; Popa, D.O.; Kang, B.H.; Lu, J.Q.; Stephanou, H.E., BCB wafer bonding compatible with bulk micro machining. Advances in Electronic Packaging 2003, Vol 1 2003, 93-99.

17. Lee, C.; Yang, E.H.; Myung, N.V.; George, T., A nanochannel fabrication technique without nanolithography. Nano Lett 2003, 3, 1339-1340.

18. Jin, J.; Wang, X.D.; Li, X.; Li, X.J.; Di, S., Nano/microchannel fabrication based on SU-8 using sacrificial resist etching method. Micro Nano Lett 2012, 7, 1320-1323.

19. Zevenbergen, M.A.G.; Wolfrum, B.L.; Goluch, E.D.; Singh, P.S.; Lemay, S.G., Fast Electron-Transfer Kinetics Probed in Nanofluidic Channels. J Am Chem Soc 2009, 131, 11471-11477.

20. Kang, S.; Nieuwenhuis, A.; Mathwig, K.; Mampallil, D.; Lemay, S.G., Electrochemical Single-Molecule Detection in Aqueous Solution Using SelfAligned Nanogap Transducers. Acs Nano 2013, 7, 10931-10937.

21. Kang, S.; Mathwig, K.; Lemay, S.G., Response time of nanofluidic electrochemical sensors. Lab on a Chip 2012, 12, 1262-1267.

22. Katelhon, E.; Krause, K.J.; Singh, P.S.; Lemay, S.G.; Wolfrum, B., Noise Characteristics of Nanoscaled Redox-Cycling Sensors: Investigations Based on Random Walks. J Am Chem Soc 2013, 135, 8874-8881.

23. Mathwig, K.; Mampallil, D.; Kang, S.; Lemay, S.G., Electrical Cross-Correlation Spectroscopy: Measuring Picoliter-per-Minute Flows in Nanochannels. Phys Rev Lett 2012, 109.

24. Lima, R.S.; Leao, P.A.G.C.; Monteiro, A.M.; Piazzetta, M.H.D.; Gobbi, A.L.; Mazo, L.H.; Carrilho, E., Glass/SU-8 microchip for electrokinetic applications. Electrophoresis 2013, 34, 2996-3002.

25. Hansen, J.S.; Dyre, J.C.; Daivis, P.J.; Todd, B.D.; Bruus, H., Nanoflow hydrodynamics. Phys Rev E 2011, 84.

26. Hanssen, B.L.; Siraj, S.; Wong, D.K.Y., Recent strategies to minimise fouling in electrochemical detection systems. Rev Anal Chem 2016, 35, 1-28.

27. Harreither, W.; Trouillon, R.; Poulin, P.; Neri, W.; Ewing, A.G.; Safina, G., Carbon Nanotube Fiber Microelectrodes Show a Higher Resistance to Dopamine Fouling. Anal Chem 2013, 85, 7447-7453.

28. Wolfrum, B.; Zevenbergen, M.; Lemay, S., Nanofluidic redox cycling amplification for the selective detection of catechol. Anal Chem 2008, 80, 972-977.

29. Katelhon, E.; Hofmann, B.; Lemay, S.G.; Zevenbergen, M.A.G.; Offenhausser, A.; Wolfrum, B., Nanocavity Redox Cycling Sensors for the Detection of Dopamine Fluctuations in Microfluidic Gradients. Anal Chem 2010, 82, 8502-8509.

51. Ilinoiu, E.C.; Manea, F.; Serra, P.A.; Pode, R., Simultaneous/Selective Detection of Dopamine and Ascorbic Acid at Synthetic Zeolite-Modified/Graphite-Epoxy Applications. 



\section{Electron transfer mediated by surface-tethered redox groups in nanogaps}

Electrochemistry provides a powerful sensor transduction and amplification mechanism that is highly suited for use in integrated, massively parallelized assays. Here we demonstrate the cyclic voltammetric detection of flexible, linear poly(ethylene glycol) polymers that have been functionalized with redox-active ferrocene (Fc) moieties and surface-tethered inside a nanofluidic device consisting of two microscale electrodes separated by a gap of $<100 \mathrm{~nm}$. Diffusion of the surfacebound polymer chains in the aqueous electrolyte allows the redox groups to repeatedly shuttle electrons from one electrode to the other, resulting in a greatly amplified steady-state electrical current. Variation of the polymer length provides control over the current, as the activity per Fc moiety appears to depend on the extent to which the polymer layers of the opposing electrodes can interpenetrate each other and thus exchange electrons. These results outline the design rules for sensing devices that are based on changing the polymer length, flexibility and/or diffusivity by binding an analyte to the polymer chain. Such a nanofluidic enabled configuration provides an amplified and highly sensitive alternative to other electrochemical detection mechanisms.

The contents of this chapter have been submitted as Tom Steentjes*, Sahana Sarkar*, Pascal Jonkheijm, Serge G. Lemay and Jurriaan Huskens, Electron transfer mediated by surface-tethered redox groups in nanofluidic devices (*equal contribution). 


\subsection{Introduction}

Biosensing plays an increasingly important role, in particular in the development of personalized medicine. ${ }^{1}$ This growing importance also puts new demands on the performance of such devices, which ranges from higher sensitivities and selectivities, to the use of smaller sample volumes, the use of body fluids, an easy readout and signal processing, and the option to use disposable devices. Electrochemical devices have particular advantages that meet several of these requirements. ${ }^{2}$ The combination with electrochemistry opens pathways for a multitude of benefits for biosensing strategies for their use in personalized medicine. ${ }^{3-5} \mathrm{~A}$ key opportunity offered by electrochemistry is an enhanced sensitivity based on signal amplification, a single molecular recognition event.

Prime examples of sensitive electrochemical detection are redox-activated DNA (EDNA) biosensors, which rely on conformational changes that modulate the motion and the distance of the redox moiety to an electrode, resulting in a change in signal. ${ }^{6-}$ ${ }^{8}$ By default, the nature of the linker and the surface density of the redox-labeled polymer chains play a pivotal role in the optimization of the sensitivity of such a sensor. Long linear molecular chains such as synthetic polymers ${ }^{9-11}$ and DNA strands end-capped with an electrochemically active label ${ }^{12-14}$ have been shown to give faradaic currents dictated by the diffusional motion of the redox moiety. This is in contrast with surface immobilized systems where the redox center is located at a fixed distance from the surface and the electron transfer rate depends exponentially on the distance to the surface. ${ }^{15-17}$

A further increase in sensitivity can be achieved by the use of electrochemical mediators which replenish the oxidized surface layer. ${ }^{18}$ Reactivation of the diffusing species in the presence of a second electrode, such as in scanning electrochemical microscopy (SECM) and nanofluidic devices, ${ }^{19}$ providing an opposing reducing or oxidizing potential, show a significant amplification of the faradaic current due to redox cycling, and this concept has been exploited for biodetection with increased sensitivity. ${ }^{20-21}$ Alternatively, if the second electrode is sufficiently close to the surface to be in contact with the electrochemically active layer, the electrode can take the role of the mediator by replenishing the oxidized/reduced species directly. Such a strategy has been employed using a conductive atomic force microscopy (AFM) tip positioned inside poly(ethylene glycol) $(P E G)^{22}$ and $D^{23} A^{23}$ layers which directly 
measures the redox cycling current provided by the bounded diffusion of the electrode-attached redox labels.

Nanodevices with nanometric electrode spacings, comparable to the molecular lengths of the surface-attached polymer chains, provide an excellent basis for electrochemical devices in which electron shuttling can occur in the absence of a mediator. This approach further promises the well-controlled and reproducible architecture of a closed solid-state device as well as integration with microfluidics, sample handling, and signal processing. ${ }^{24}$ Several device architectures have been demonstrated that lend themselves to miniaturization down to the nanometer scale, including interdigitated electrode arrays, ${ }^{25-27}$ recessed ring electrodes ${ }^{28}$ and thinlayer cells. ${ }^{19,}$, $9-30$ As of yet, however, no such system has been reported in a device configuration where surface attached redox couples are directly replenished due to the effect of the second electrode without the need of a mediator.

Herein we report nanofluidic devices with two opposing microscale electrodes spaced by a sub-100 nm gap and their functionalization with electrochemically active end-capped polymers in order to study their ability to transfer electrons between the electrodes across the nanogap in the absence of a mediator. We compare the effects of different polymer length and of the length distribution, as well as the effect of changes in surface density on the measured current.

\subsection{Methods}

Materials. Reagents and solvents were purchased from Sigma-Aldrich, high-purity water (MilliQ) was used (Millipore, $\mathrm{R}=18.2 \mathrm{M} \Omega$ ). All bis-NHS-functionalized PEGs were purchased from Nanocs and have a reported dispersity of 1.08 .

Device fabrication. A 4-inch Si wafer was isolated with $500 \mathrm{~nm}$ thick thermally grown $\mathrm{SiO}_{2}$. A Pt bottom electrode, a $\mathrm{Cr}$ sacrificial layer and a $\mathrm{Pt}$ top electrode were then consecutively deposited by electron-beam evaporation of $20 \mathrm{~nm}, 60 \mathrm{~nm}$ and $100 \mathrm{~nm}$ of metal, respectively, and patterned using a lift-off process based on a positive photoresist (OIR 907-17, Arch Chemicals). Thereafter, a passivation layer consisting of $90 \mathrm{~nm} / 325 \mathrm{~nm} / 90 \mathrm{~nm}$ thick PECVD SiO$/ 2 / \mathrm{Si}_{3} \mathrm{~N}_{4} / \mathrm{SiO}_{2}$ was deposited. Access holes were then etched through the passivation layer in by reactive ion etching, reaching the $\mathrm{Cr}$ sacrificial layer. Lastly, the sacrificial layer was etched by placing a drop of chromium etchant (BASF, Chromium Etch Selectipur) at the inlets of the 
nanochannel. Additional details of the fabrication process have been described elsewhere. ${ }^{36-37}$

Electrochemical experiments in the nanodevice. The electrodes were cleaned prior to the measurements by cycling their potential in $0.5 \mathrm{M} \mathrm{H}_{2} \mathrm{SO}_{4}$ between -0.15 and $1.2 \mathrm{~V}$ at $50 \mathrm{mV} / \mathrm{s}$ until a stable voltammogram was obtained. The devices were rinsed with milliQ water and a polydimethylsiloxane (PDMS) reservoir was positioned above the device which and was filled with a $2 \mathrm{mM}$ solution of cystamine and left overnight. Subsequently, the devices were rinsed with a copious amount of water and filled with a $0.2 \mathrm{mM}$ Fc-PEG-NHS solution in water for $3 \mathrm{~h}$ or the incubation times indicated in Figure 6.5, after which the device was immersed in a beaker with milliQ water which was vigorously stirred for at least 5 min (control measurements have shown that this

a)

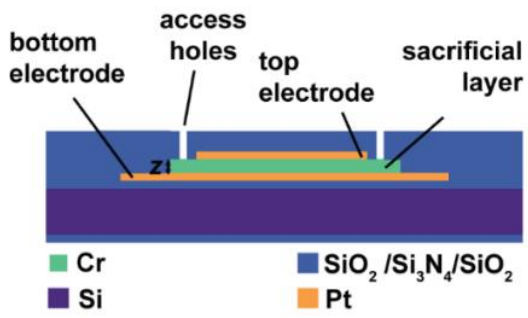

b)

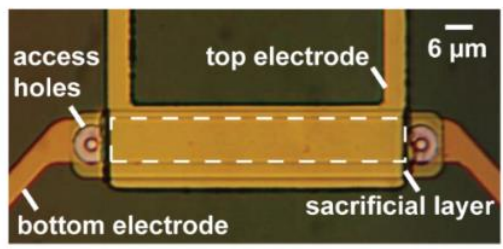

c)

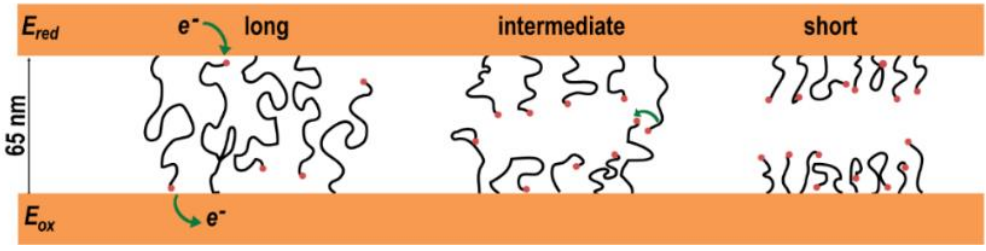

Figure 6.1. (a) Schematic diagram of the cross-section of the nanodevice prior to sacrificial layer etching (not drawn to scale), where $z=65 \mathrm{~nm}$ represents the height of the nanogap. (b) Optical microscopy image of the top view of a Type II device before the sacrificial layer was etched. The dotted white rectangle represents the active area. (c) The nanogap electrodes were functionalized with one of three different ferrocene end-capped PEG chains. A "long" PEG (here: PEG ${ }_{10 k}$ ) provides sufficient length to bridge the gap between the two electrodes so it can cycle electrons between the two electrodes. The "intermediate" PEG (here: $\mathrm{PEG}_{5 k}$ ) is too short to span the full gap but is long enough for the ferrocene groups of the opposing electrodes to exchange electrons. A "short" PEG (here: PEG ${ }_{3 k}$ ) is too short to reach even half way and therefore a negligible current is expected. 
is sufficient time to remove all physisorbed PEG chains from the channel, see Appendix C, Figure C1). Before measurements, the devices were filled with a $1 \mathrm{M}$ $\mathrm{NaClO}_{4}$ supporting electrolyte solution, and a standard $\mathrm{Ag} / \mathrm{AgCl}$ electrode (BASi, MF 2079, RE-5B) inserted in the PDMS reservoir was used as the reference electrode. No counter electrode was used as the current through the reference is minimal in this configuration. Two transimpedance amplifiers (Femto, DDPCA-300) were used to apply potentials to the two working electrodes with respect to the reference electrode and monitor the currents through these electrodes.

\subsection{Results and discussion}

The nanogap devices used here (Figure 6.1a) consist of two planar rectangular platinum electrodes that are separated by a distance of $65 \mathrm{~nm}$. To fabricate the nanofluidic channel, a sacrificial chromium layer was sandwiched between two platinum electrodes followed by wet etching of the chromium to open up the nanochannel. Two access holes acted as the (diffusive) inlets to the nanochannel. The active region for redox cycling is defined by the overlapping area of the two electrodes, as indicated in Figure 6.1b. Two different types of nanofluidic devices were used (Type I and Type II), the devices differing primarily in the dimensions of the areas of the active region ( $30 \mu \mathrm{m}^{2}$ for Type I and $300 \mu \mathrm{m}^{2}$ for Type II). In practice both device types yielded equivalent results, as described further below.

The platinum electrodes were modified with cystamine, resulting in a monolayer with exposed amine groups. On this monolayer, bifunctional PEG chains were grafted via a reactive succinimide group on one end of the chain. The other end bore a redoxactive ferrocene label which was used for electrochemical detection. Three lengths of PEG, "long", "intermediate" and "short", were used in this study to probe the possible occurrence of three different electrochemical regimes, as shown in Figure 6.1c. The "long" PEG $10 \mathrm{k}\left(M_{n}=10 \pm 2.8 \mathrm{kDa}\right.$; single standard deviation, polydispersity index PDI $=1.08$ ) with an average, fully extended chain length $L$ of $79 \mathrm{~nm}$ is longer than the spacing between the electrodes, making it possible for the ferrocene endgroups to move between the two electrodes through bounded diffusion supplied by the flexible polymer backbone and shuttle electrons. The "intermediate" PEG ${ }_{5 k}\left(M_{n}=\right.$ $5.0 \pm 1.4 \mathrm{kDa}$ ) with $L=40 \mathrm{~nm}$ is too short to reach the opposing electrode, but is still long enough to reach halfway and meet the ferrocene head groups tethered to the other electrode, whereas the "short" PEG $3 \mathrm{k}\left(M_{n}=3.4 \pm 0.96 \mathrm{kDa}\right)$ with $L=27 \mathrm{~nm}$ is in principle too short for both the above mentioned scenarios. 
The nanogap electrodes were initially functionalized with PEG for at least $3 \mathrm{~h}$ in order to obtain maximum surface densities. To make sure that all physisorbed PEG molecules were removed from the electrodes, the device was held in a beaker with milliQ water and vigorously stirred for at least $10 \mathrm{~min}$. Without this procedure, the redox signal decreased during flow, while the rinsing procedure resulted in signals that were stable for the duration of the experiments. Additionally, control experiments using mercaptoethanol instead of cystamine - thus prohibiting the attachment of the Fc-modified PEG - showed complete removal of redox activity upon applying the same rinsing method (Appendix C, Figure C1), and thus confirmed the suitability of this method.

In order to measure the surface densities at the electrodes, the top and bottom electrodes were connected together and cycled as one electrode as shown in Figure 6.2a. ${ }^{31}$ The results are illustrated in Figure 6.2b, which shows a cyclic voltammogram measured on a Type I device grafted with PEG $_{10 k}$. The recorded voltammogram is characteristic for surface-attached species, with a peak separation less than $59 \mathrm{mV}$ for all scan rates employed, a formal potential $\left(E_{0}^{\prime}\right)$ of $0.3 \mathrm{~V} v \mathrm{~s}$. $\mathrm{Ag} / \mathrm{AgCl}$ and a peak current $(/ p)$ which increases linearly with the scan rate (Figure $6.2 \mathrm{c}$ ). ${ }^{32}$ For surfaceattached species, the charge under the peak is proportional to the total number of

a)

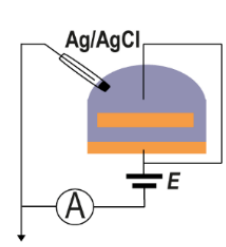

b)

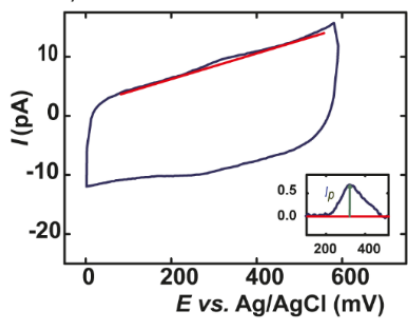

c)

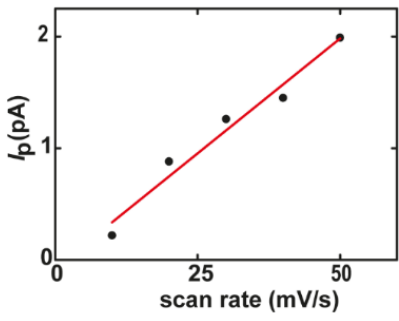

Figure 6.2. (a) Schematic diagram of the measurement configuration when both electrodes are cycled together as one electrode. (b) Cyclic voltammogram of ferrocene end-capped $\mathrm{PEG}_{10 \mathrm{k}}$ functionalized electrodes measured in a Type I device at both electrodes simultaneously in a $1 \mathrm{M} \mathrm{NaClO}_{4}$ solution (scan rate $20 \mathrm{mV} \mathrm{s}^{-1}$ ). The inset shows the baseline-corrected anodic peak, the area of which was integrated to extract

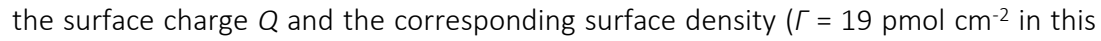
case). (c) Plot of the peak current vs. scan rate, indicating a linear increase of the peak current with the scan rate. 
electrons transferred and can be used to calculate the surface density $\Gamma$ with $\Gamma=$ $Q / n F\left(A_{\text {top }}+A_{\text {bottom }}\right)$. Here $Q$ is the measured charge (as shown in Figure 6.2b), $n$ the number of electrons transferred per ferrocene moiety $(n=1), F$ the Faraday constant, and $A_{\text {top }}$ and $A_{\text {bottom }}$ are the geometric surface areas of the two electrodes exposed to

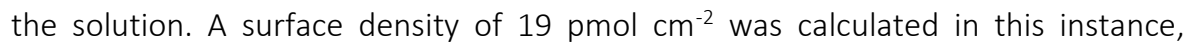
corresponding to an average spacing of $3 \mathrm{~nm}$ between the polymer chains. This value is smaller than the calculated Flory radius $\left(R_{\mathrm{f}}=9.1 \mathrm{~nm}\right)$ of the polymer coils (the value of $R_{f}$ for good solvents can be calculated from $R_{f}=a N^{3 / 5}$, where $a=0.35 \mathrm{~nm}$ is the monomer length ${ }^{33}$ and $N=227$ is the degree of polymerization). Therefore it can be concluded that the polymer was attached to the surface in a loose brush conformation, extending upwards from the electrode surface. ${ }^{22,34}$

Subsequently, the two electrodes were addressed separately: a reducing potential $(0 \mathrm{~V})$ was applied to the bottom electrode, while the potential of the top electrode was cycled between 0 and $0.6 \mathrm{~V}$ (Figure 6.3a). When the potential of the sweeping electrode passed $E_{0}{ }^{\prime}$, oxidized ferrocenium species attached to the PEG molecules could regain an electron, either by diffusion to the reducing electrode or by electron transfer from a Fc group attached to this electrode; the opposite being true for reduced species contacting the oxidizing electrode, thus giving rise to a redox cycling current. The redox cycling currents measured at the two electrodes are expected to have the same magnitude but opposite signs. The resulting cyclic voltammograms for
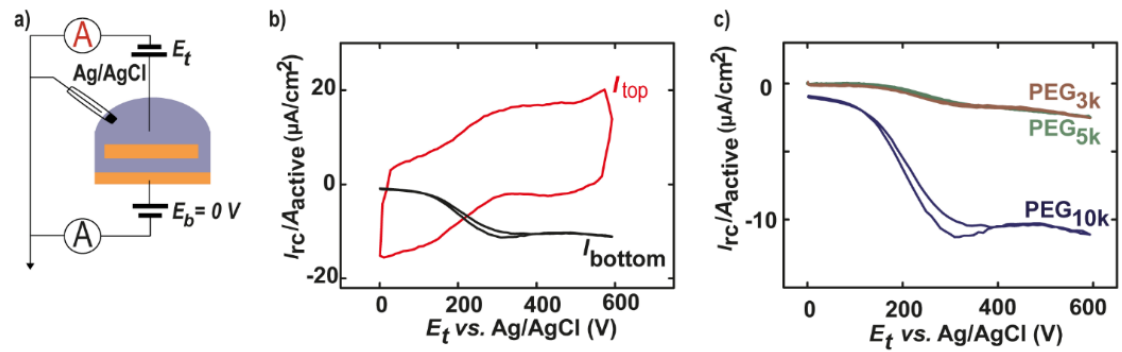

Figure 6.3. (a) Schematic diagram of the measurement configuration showing that the top electrode is cycled (red) while the bottom electrode is fixed at $0 \mathrm{~V}$ (black). (b) Simultaneously recorded voltammograms for $\mathrm{PEG}_{10 \mathrm{k}}$ (Type II device; $\Gamma=8.8 \mathrm{pmol} \mathrm{cm}{ }^{-2}$ ). (c) Comparison of the redox cycling current obtained from $\mathrm{PEG}_{10 \mathrm{k}}$ (data from panel b),

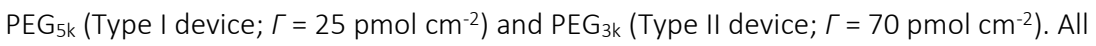
measurements were performed at a scan rate of $50 \mathrm{mV} \mathrm{s}^{-1}$ and the currents were normalized by the area $A_{\text {active }}$ to facilitate comparison. 


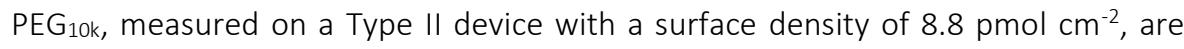
shown in Figure 6.3b, where the simultaneously measured currents through each of the two working electrodes are plotted. The current at the electrode being cycled includes a large capacitive contribution which is marked by a pronounced hysteresis (Figure 6.3b, red curve). This capacitance results in part from the electrical double layer in regions where the electrode is exposed to the solution as well as from the capacitance of the connecting wires, which have a relatively large area and are only protected from the solution by a thin passivating layer of $\mathrm{SiN} / \mathrm{SiO}_{2} / \mathrm{SiN}$. For this reason we concentrate our analysis below on the current measured at the electrode held at a fixed potential, which nicely isolates the redox cycling component.

Additionally, in order to probe the different electrochemical regimes depicted in Figure 6.1c, a Type I device was functionalized with PEG Fk $_{k}$ and a type II device with a tenfold larger active area was used for $\mathrm{PEG}_{3 \mathrm{k}}$. Figure $6.3 \mathrm{c}$ shows the obtained redox cycling currents normalized to the area of the active region, Aactive, for the three different devices. The steady state redox cycling current obtained for $P E G_{10 k}$ is nearly an order of magnitude higher than that obtained for PEG5k. This could be expected since the PEG5k chains are not long enough to reach the opposite electrode. The fact that a non-zero current is still recorded with $\mathrm{PEG}_{5 \mathrm{k}}$ indicates that electron exchange takes place between the two electrodes in the solution zone where the ferrocene ends of both molecular layers can meet. Only the ferrocene on the sweeping electrode is expected to oxidize, but since the ferrocene moieties on both electrodes can extend half-way through the nanogap, the ferrocenes attached to the opposing reducing electrode can donate electrons and act as a mediator. Since the amount of ferrocene moieties attached to the electrodes is known ( $\Gamma=25 \mathrm{pmol} \mathrm{cm}{ }^{-2}$ in this case), a concentration of $7.4 \mathrm{mM}$ ferrocene can be calculated to be present inside the active region. This is a concentration comparable to mediators used in SECM for measurements on monolayers. ${ }^{35} \mathrm{PEG}_{3 \mathrm{k}}$ is even shorter and was not expected to be able to exchange electrons as the average, fully extended length, $L=27 \mathrm{~nm}$, does not allow the ferrocene labels attached to the electrode to reach halfway across the channel. However, at the present PDI of 1.08, corresponding to a standard deviation of $0.96 \mathrm{kDa}$ (or a total of $4.4 \mathrm{kDa}$ ) the length of a significant fraction of the polymer chains can extend beyond $34 \mathrm{~nm}$, which exceeds half the gap separation. This makes it plausible that a fraction of polymer chains is capable of participating in electron transfer. Combined with the higher coverage for $\mathrm{PEG}_{3 \mathrm{k}}\left(70 \mathrm{pmol} \mathrm{cm}{ }^{-2}\right)$, this explains the observed similar current values obtained for $P E G_{5 k}$ and $P E G_{3 k}$. 
The experiments of Figures 6.2 and 6.3 were repeated in several additional devices for all three chain lengths and the results are summarized in Figure 6.4. The measured surface densities decreased with increasing polymer length (Figure 6.4a), as expected since the increased Flory radius leads to steric hindrance. Some scatter is observed in the data, which we attribute primarily to surface roughness: the area values are based on the geometric surface area and averaged over both electrodes, whereas the surface roughness is not the same for both electrodes (see Appendix C, Figure $\mathrm{C} 2$ ) and can vary between devices. The corresponding redox cycling currents were recorded simultaneously and were similar to the examples shown in Figure 6.3. To get a clear picture of the effect of the linker length, the redox cycling currents were normalized to the surface density according to $v=1 \mathrm{rc} /\left(e N_{\text {active }}\right.$ ) (with e the charge of an electron and $N_{\text {active }}$ the amount of PEG-Fc molecules in the active area) - yielding the rate of electron transfer per available ferrocene group - as presented in Figure $6.4 \mathrm{~b}$ (real currents are reported in the Appendix C, Figure C3). This analysis shows that a single $P E G_{10 k}$ chain functionalized with a ferrocene end-group is able to transfer on average 6 electrons to the opposing electrode per second, whereas the electron transfer rate drops to $1 \mathrm{~s}^{-1}$ per ferrocene for $\mathrm{PEG}_{5 \mathrm{k}}$, reflecting the decrease in polymer length and the more limited ability to reach the other electrode. It also becomes clear that, although the measured redox cycling currents for $\mathrm{PEG}_{3 k}$ are not significantly lower than for $\mathrm{PEG}_{5 k}$, the rate of electrons transferred per ferrocene available is only $0.3 \mathrm{~s}^{-1}$ for $P E G_{3 k}$, and thus the measured redox cycling currents for $\mathrm{PEG}_{3 \mathrm{k}}$ compared

a)

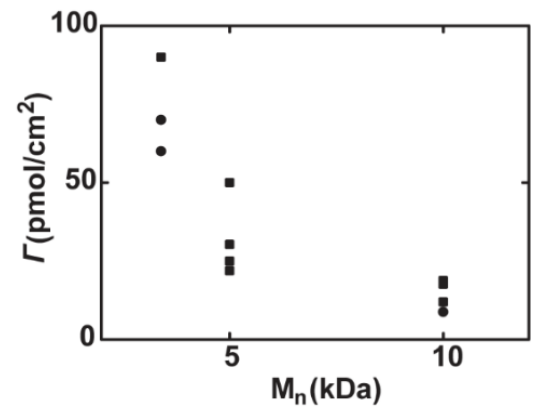

b)

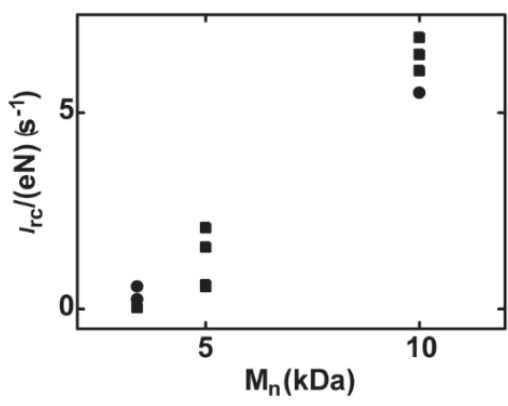

Figure 6.4. (a) The effect of linker length on the obtained maximum surface density after $3 \mathrm{~h}$ functionalization, and (b) the corresponding redox cycling currents, presented as the rate of electrons transferred per PEG chain. The measurements were performed on both types of devices, Type I = $\mathbf{\square}$, Type $I=\bullet$ 
to $\mathrm{PEG}_{5 k}$ are the result of a higher surface density while a smaller fraction of the polymer chains is long enough to contribute to electron transfer.

When considering the mechanism by which the electrons are transferred, it was anticipated that the polymer length of $P E G_{10 k}$ is long enough to enable the attached ferrocene moiety to travel to the opposing electrode, whereas the chain length for $P G_{5 k}$ can only provide for electron transfer between the ferrocene moieties itself inside the channel. Not taking into account the dispersity of the polymers, the length of $40 \mathrm{~nm}$ of PEG5k gives rise to a $15 \mathrm{~nm}$ exchange zone inside the $65 \mathrm{~nm}$ channel where the polymer chains from both electrodes can overlap and the ferrocene moieties can exchange electrons. For the $\mathrm{PEG}_{10 \mathrm{k}}$ chains to reach the opposing electrodes they have to stretch outwards and penetrate through the polymer layer resulting in an exchange zone of $65 \mathrm{~nm}$ (the entire channel). Next to larger exchange zone by a factor 4 , the concentration of ferrocene inside the channel is on average a factor 2 lower for $\mathrm{PEG}_{10 \mathrm{k}}$ than for $\mathrm{PEG}_{5 \mathrm{k}}$ due to the lower surface densities, which corresponds to an overall expected higher activity of a factor 2 for PEG $10 \mathrm{k}$. Additionally, for $\mathrm{PEG}_{10 \mathrm{k}}$ there is no need to stretch fully to reach an opposing PEG chain. Taking all these factors into account, we attribute the observed 5-fold higher activity of $P E_{10 k}$ compared to $P E G_{5 k}$ to electron transfer dominated by exchange between the diffusing and interpenetrating ferrocene moieties in both cases, rather than direct transfer to the opposing electrode. We expect the same mechanism to hold for $\mathrm{PEG}_{3 \mathrm{k}}$ as well, when taking the dispersity into account. In this case, only a fraction of the molecules is sufficiently long to allow some interpenetration of the chain ends from opposing electrodes which, combined with the higher surface density, leads to the observed 3 times lower activity compared to PEG 5 .

When taking the polymer chain length dispersities into account for all polymers, the interpenetration and exchange zones do not have sharply defined boundaries, but the exchange extends outside of the given borders with decreasing probabilities. Since proper electron transfer rate calculations would have to take into account the polymer length distributions, probability distributions of the distance between the Fc end group and the electrode, collision frequencies, polymer surface densities, diffusion rates, and $\mathrm{Fc}-\mathrm{Fc}$ and Fc-electrode electron transfer rates, we did not attempt this here. From the almost linear behavior observed for the electron transfer per Fc moiety for the polymers studied here (Figure 6.4b) and the arguments given above, we conclude that the interpenetration and Fc-Fc exchange model (as depicted for the "intermediate" case in Figure 6.1c) holds for all polymer lengths studied here. 
The conformation of polymers grafted to the surface depends on the surface density, with the polymers existing in a mushroom conformation at low surface densities, where the polymers are isolated from their neighboring chains, and becoming more brush-like with increasing surface density as the surface concentration passes a critical surface density. ${ }^{34}$ In order to study a possible effect of the PEG density and conformation regime on the redox cycling currents, Type I devices were functionalized with different surface densities of $P E G_{10 k}$, by changing the time the molecules were allowed to react with the cystamine monolayer. The resulting redox cycling currents were plotted vs. the incubation time, as shown in Figure 6.5. The surface densities, especially for short reaction times, were too low to give clearly distinguishable peaks in the cyclic voltammograms and no reliable surface densities could be recorded. Therefore the redox cycling currents were compared with surface densities determined from experiments on macro electrodes. As can be seen in Figure 6.5a, the obtained surface densities on the macro electrodes reach a plateau around $40 \mathrm{pmol} \mathrm{cm}$, which is more than a factor 2 higher than the values obtained inside the nanogap devices (Figure 6.4a). Therefore it is expected that the surface densities inside the nanogap devices are still in the linear regime (left side of Figure 6.5a) for all incubation times used, probably due to slower mass transport compared to the macro electrodes. Indeed, it can be seen (Figure 6.5b) that the recorded redox cycling currents increase linearly with the incubation time. It can thus be concluded that the increase in current with increasing reaction times is mostly the result of the increase of ferrocene density and not of a change in polymer conformation. Due to the bulky nature of the $\mathrm{PEG}_{10 \mathrm{k}}$ molecule, all used reaction times result in polymers present in a loose brush conformation (the maximum surface density for the

a)

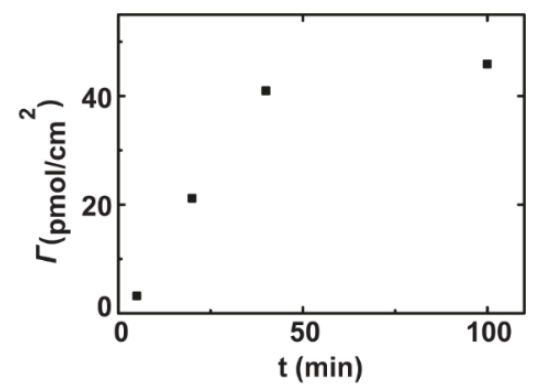

b)

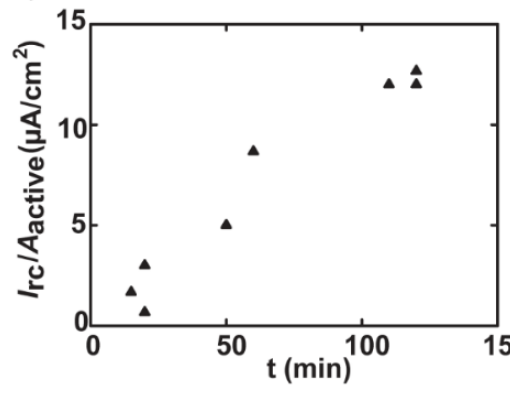

Figure 6.5. (a) Surface densities on macro electrodes, and (b) redox cycling currents in nanogap devices for $\mathrm{PEG}_{10 \mathrm{k}}$ as a function of the incubation time. 
mushroom configuration for $\mathrm{PEG}_{10 \mathrm{k}}$ is calculated as $\left.2 \mathrm{pmol} \mathrm{cm}{ }^{-2}\right),{ }^{34}$ and no dramatic change of behavior could be observed.

Capacitive charging. A different manifestation of the polymer-mediated charge transport between the electrodes based on capacitive charging was also observed. In systems where the spacing between two electrodes is at the macro/micro scale, it is possible to determine the coverage at the individual electrodes by sweeping each electrode independently. However, within nanogaps, owing to the small separation, the electrodes cannot be swept independently, as has been addressed at length in Chapter 4. In short, electroactive species present within the nanogap are capable of redox cycling as long as there exists a potential difference between the two electrodes. Thus, if one of the electrodes is biased and the other is left floating, ferrocenium species attached to the PEG molecules are capable of bridging and thus undergo redox cycling as long as there exists a potential difference between the two electrodes (Figure 6.6a). This redox cycling continues until the two electrodes reach the same potential. Consequently, the potential of the floating electrode changes and this process can be monitored. In order to demonstrate this, we conducted experiments by sweeping only one electrode at a time while monitoring the potential at the floating electrode. These measurements were conducted in Type II devices and

(a)

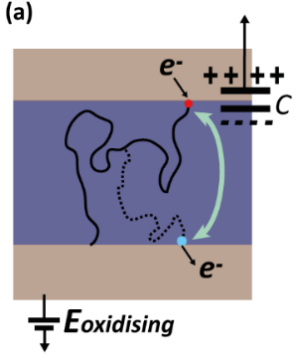

(b)

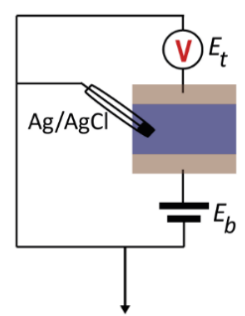

(c)

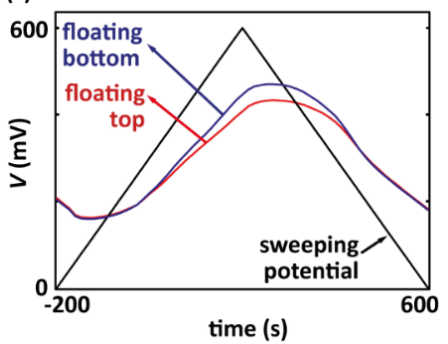

Figure 6.6. (a) Schematic diagram illustrating the capacitive charging effect: a redox moiety end-tethered on a longer polymer and attached on the surface of an electrode shuttles charge to the second, unbiased floating electrode. Double layer capacitance at the floating electrode, $C$, accumulates charge over time as the redox moiety undergoes redox cycling, thereby developing a potential. b) Experimental setup where the bottom electrode is swept and the potential of the floating top electrode is monitored. c) The triangular black curve shows the sweeping potential in each case while the red and blue curves show the potential observed when the top and bottom electrodes are allowed to float, respectively. 
PEG 10k, capable of reaching both electrodes, was chosen to facilitate redox cycling. Figure $6.6 \mathrm{~b}$ shows the equivalent circuit diagram of the experimental setup where the bottom electrode was swept while the potential of the floating top electrode was measured using an electrometer (Keithley model 617). The two cases, one in which the top electrode was swept and the bottom electrode potential was monitored (shown in blue, $V_{p p}=302 \mathrm{mV}$ ), and vice versa (shown in red, $V_{p p}=278 \mathrm{mV}$ ), are shown in Figure 6.6c. In both cases the sweeping electrode is swept at a potential range between 0 to $600 \mathrm{mV}$ (represented by the black curve). As expected, significant tracking of the potential of the controlled electrode by the floating electrode is observed in both cases.

For comparison, the surface coverage determined by cycling the top and bottom electrodes together $\left(\Gamma=Q / n F\left(A_{\text {top }}+A_{\text {bottom }}\right)\right)$ and was found to be about $\sim 8 \mathrm{pmol} / \mathrm{cm}^{2}$. However, on sweeping the electrodes individually as described in this section, the surface coverage that would have been found ignoring the electrode coupling would have been $39 \mathrm{pmol} / \mathrm{cm}^{2}$ and $25 \mathrm{pmol} / \mathrm{cm}^{2}$ at the top and bottom electrodes, respectively. This motivates why it was chosen to sweep both electrodes simultaneously to evaluate surface coverage instead of sweeping the electrodes individually. On the other hand, it provides an additional illustration of how tethered redox moieties can effectuate coupling between electrodes with a nanoscale spacing.

\subsection{Conclusion}

We have shown that surface-tethered PEG polymer chains with redox-active end groups allow the transfer of electrons between the electrodes in nanogap devices in the absence of a freely-diffusing electrochemical mediator. The primary condition that needs to be met is that the polymer chains of the opposing electrodes can interpenetrate so that oxidized and reduced ferrocene moieties can exchange electrons. The activity per Fc unit, as defined by the average number of electrons transferred per Fc moiety, is strongly dependent on the chain length, but not on the chain density in the loose brush density range studied here. The chain length effect is interpreted primarily by the increasing thickness of the channel zone in which exchange can occur and the increasing fraction of the polymers that can reach this exchange zone. The overall measured cycling currents are additionally dependent on the surface density, which is lower for longer polymers and thus counteracts to some extent the enhanced activities observed for the longer PEGs. All polymer lengths 
studied here were found to obey this same interpenetration and electron exchange model.

The findings of this study can contribute to an enhanced understanding and improved performance of biosensing devices that use redox cycling in surface-tethered receptor devices as the main amplification mechanism, such as in DNA hairpin-like detection schemes. An increase in current, and with that an increase in sensitivity, can be expected if the height of the nanochannel is more finely tuned towards the length of the linker. At the same time, the selectivity between bound and unbound receptors can be improved when the polymer length distribution is more narrow and when the extended chain length is close to half of the channel width, so that small changes in polymer stiffness and length have large effects on the probabilities of chain interpenetration and electron exchange.

\section{$6.5 \quad$ References}

1. Hamburg, M.A.; Collins, F.S., The Path to Personalized Medicine. N. Engl. J. Med. 2010, 363, 301-304.

2. Wang, J., Electrochemical biosensors: Towards point-of-care cancer diagnostics. Biosens. Bioelectron. 2006, 21, 1887-1892.

3. Kirsch, J.; Siltanen, C.; Zhou, Q.; Revzin, A.; Simonian, A., Biosensor technology: Recent advances in threat agent detection and medicine. Chem. Soc. Rev. 2013, 42, 8733-8768.

4. Perumal, V.; Hashim, U., Advances in biosensors: Principle, architecture and applications. J. Appl. Biomed. 2014, 12, 1-15.

5. Rackus, D.G.; Shamsi, M.H.; Wheeler, A.R., Electrochemistry, biosensors and microfluidics: a convergence of fields. Chem. Soc. Rev. 2015, 44, 5320-5340.

6. Fan, C.; Plaxco, K.W.; Heeger, A.J., Electrochemical interrogation of conformational changes as a reagentless method for the sequence-specific detection of DNA. Proc. Natl. Acad. Sci. U. S. A. 2003, 100, 9134-9137.

7. Xiao, Y.; Qu, X.; Plaxco, K.W.; Heeger, A.J., Label-free electrochemical detection of DNA in blood serum via target-induced resolution of an electrode-bound DNA pseudoknot. J. Am. Chem. Soc. 2007, 129, 11896-11897.

8. Xiao, Y.; Lou, X.; Uzawa, T.; Plakos, K.J.I.; Plaxco, K.W.; Soh, H.T., An Electrochemical Sensor for Single Nucleotide Polymorphism Detection in Serum Based on a Triple-Stem DNA Probe. J. Am. Chem. Soc. 2009, 131, 15311-15316.

9. Anne, A.; Demaille, C.; Moiroux, J., Terminal attachment of polyethylene glycol (PEG) chains to a gold electrode surface. Cyclic voltammetry applied to the quantitative characterization of the flexibility of the attached PEG chains and of their penetration by mobile PEG chains. Macromolecules 2002, 35, 5578-5586. 
10. Anne, A.; Moiroux, J., Quantitative characterization of the flexibility of poly(ethylene glycol) chains attached to a glassy carbon electrode. Macromolecules 1999, 32, 5829-5835.

11. Blauch, D.N.; Saveant, J.M., Dynamics of electron hopping in assemblies of redox centers. Percolation and diffusion. J. Am. Chem. Soc. 1992, 114, 3323-3332.

12. Anne, A.; Bouchardon, A.; Moiroux, J., 3'-ferrocene-labeled oligonucleotide chains end-tethered to gold electrode surfaces: Novel model systems for exploring flexibility of short DNA using cyclic voltammetry. J. Am. Chem. Soc. 2003, 125, 1112-1113.

13. Huang, K.C.; White, R.J., Random walk on a leash: A simple single-molecule diffusion model for surface-tethered redox molecules with flexible linkers. J. Am. Chem. Soc. 2013, 135, 12808-12817.

14. Hüsken, N.; Gębala, M.; La Mantia, F.; Schuhmann, W.; Metzler-Nolte, N., Mechanistic studies of Fc-PNA(-DNA) surface dynamics based on the kinetics of electron-transfer processes. Chem. - Eur. J. 2011, 17, 9678-9690.

15. Smalley, J.F.; Feldberg, S.W.; Chidsey, C.E.D.; Linford, M.R.; Newton, M.D.; Liu, Y.-P., The Kinetics of Electron Transfer Through Ferrocene-Terminated Alkanethiol Monolayers on Gold. J. Phys. Chem. 1995, 99, 13141-13149.

16. Weber, K.; Hockett, L.; Creager, S., Long-Range Electronic Coupling between Ferrocene and Gold in Alkanethiolate-based Monolayers on Electrodes. J. Phys. Chem. B 1997, 101, 8286-8291.

17. Finklea, H.O.; Hanshew, D.D., Electron-transfer kinetics in organized thiol monolayers with attached pentaammine(pyridine)ruthenium redox centers. $J$. Am. Chem. Soc. 1992, 114, 3173-3181.

18. Merchant, S.A.; Meredith, M.T.; Tran, T.O.; Brunski, D.B.; Johnson, M.B.; Glatzhofer, D.T.; Schmidtke, D.W., Effect of Mediator Spacing on Electrochemical and Enzymatic Response of Ferrocene Redox Polymers. J. Phys. Chem. C 2010, $114,11627-11634$.

19. Zevenbergen, M.A.G.; Krapf, D.; Zuiddam, M.R.; Lemay, S.G., Mesoscopic concentration fluctuations in a fluidic nanocavity detected by redox cycling. Nano Lett. 2007, 7, 384-388.

20. Zhao, C.; Wittstock, G., An SECM Detection Scheme with Improved Sensitivity and Lateral Resolution: Detection of Galactosidase Activity with Signal Amplification by Glucose Dehydrogenase. Angew. Chem. Int. Ed. 2004, 43, 41704172.

21. Rassaei, L.; Mathwig, K.; Kang, S.; Heering, H.A.; Lemay, S.G., Integrated biodetection in a nanofluidic device. ACS Nano 2014, 8, 8278-8284.

22. Abbou, J.; Anne, A.; Demaille, C., Probing the structure and dynamics of endgrafted flexible polymer chain layers by combined atomic force-electrochemical microscopy. Cyclic voltammetry within nanometer-thick macromolecular poly(ethylene glycol) layers. J. Am. Chem. Soc. 2004, 126, 10095-10108. 
23. Wang, K.; Goyer, C.; Anne, A.; Demaille, C., Exploring the motional dynamics of end-grafted DNA oligonucleotides by in situ electrochemical atomic force microscopy. J. Phys. Chem. B 2007, 111, 6051-6058.

24. Rassaei, L.; Singh, P.S.; Lemay, S.G., Lithography-Based Nanoelectrochemistry. Anal. Chem. 2011, 83, 3974-3980.

25. Dam, V.A.T.; Olthuis, W.; van den Berg, A., Redox cycling with facing interdigitated array electrodes as a method for selective detection of redox species. Analyst 2007, 132, 365-370.

26. Goluch, E.D.; Wolfrum, B.; Singh, P.S.; Zevenbergen, M.A.G.; Lemay, S.G., Redox cycling in nanofluidic channels using interdigitated electrodes. Anal Bioanal Chem 2009, 394, 447-456.

27. Samarao, A.K.; Rust, M.J.; Ahn, C.H. In Rapid fabrication of a nano interdigitated array electrode and its amperometric characterization as an electrochemical sensor, Proc. IEEE Sens., 2007; pp 644-647.

28. Ma, C.; Contento, N.M.; Gibson, L.R.; Bohn, P.W., Redox cycling in nanoscalerecessed ring-disk electrode arrays for enhanced electrochemical sensitivity. ACS Nano 2013, 7, 5483-5490.

29. Kätelhön, E.; Hofmann, B.; Lemay, S.G.; Zevenbergen, M.A.G.; Offenhäusser, A.; Wolfrum, B., Nanocavity redox cycling sensors for the detection of dopamine fluctuations in microfluidic gradients. Anal. Chem. 2010, 82, 8502-8509.

30. Xiong, J.; Chen, Q.; Edwards, M.A.; White, H.S., Ion Transport within High Electric Fields in Nanogap Electrochemical Cells. ACS Nano 2015, 9, 8520-8529.

31. Oleinick, A.I.; Battistel, D.; Daniele, S.; Svir, I.; Amatore, C., Simple and Clear Evidence for Positive Feedback Limitation by Bipolar Behavior during Scanning Electrochemical Microscopy of Unbiased Conductors. Anal. Chem. 2011, 83, 4887-4893.

32. Bard, A.J.; Faulkner, L.R., Electrochemical Methods: Fundamentals and Applications. 2 ed.; John Wiley \& Sons: New York, 2001.

33. Allen, C., et al., Controlling the physical behavior and biological performance of liposome formulations through use of surface grafted poly(ethylene glycol). Biosci. Rep. 2002, 22, 225-250.

34. De Gennes, P.G., Conformations of polymers attached to an interface. Macromolecules 1980, 13, 1069-1075.

35. Liu, B.; Bard, A.J.; Mirkin, M.V.; Creager, S.E., Electron Transfer at Self-Assembled Monolayers Measured by Scanning Electrochemical Microscopy. J. Am. Chem. Soc. 2004, 126, 1485-1492. 


\section{Appendix A}

\section{A.1 Fabrication of nanogap electrodes}

The method for microfabricating the nanogap devices is summarized here. A platinum electrode, $20 \mathrm{~nm}$ thick, bottom electrode was deposited using electron beam evaporation using a Blazers BAK 600 system and patterned by photolithography and a lift off process using Arch Chemical OIR 907-17 as photoresist. Thereafter, a chromium layer, $60 \pm 5 \mathrm{~nm}$, was deposited using the same technique as the sacrificial layer. A $100 \mathrm{~nm}$ platinum top electrode was also subsequently deposited and patterned using the same procedure. The whole structure was buried in a passivation layer of $750 \mathrm{~nm}$ silicon nitride employing a Film PECVD Oxford 80 system. Using reactive ion etching (Etch RIE Plasma Therm 790), two access holes, $2 \times 2 \mu \mathrm{m}$, were then etched through the passivation layer to allow the inflow of solution into the nanochannel. The top and cross sectional view of the device is sketched in Figure A1.

\section{A.2 Characterization of solution after exposure to device}

$1 \mathrm{mM}$ solution of Ferrocenedimethanol $\left(\mathrm{Fc}(\mathrm{MeOH})_{2}\right.$, Sigma-Aldrich, Catalog no. 372625 , diffusion coefficient $D=6.7 \times 10^{-10} \mathrm{~m}^{2} / \mathrm{s}$ ), nominally in the reduced form, was prepared in Milli-Q water with $0.1 \mathrm{M} \mathrm{KCl}$ added as supporting electrolyte. Cyclic 


\section{top view}

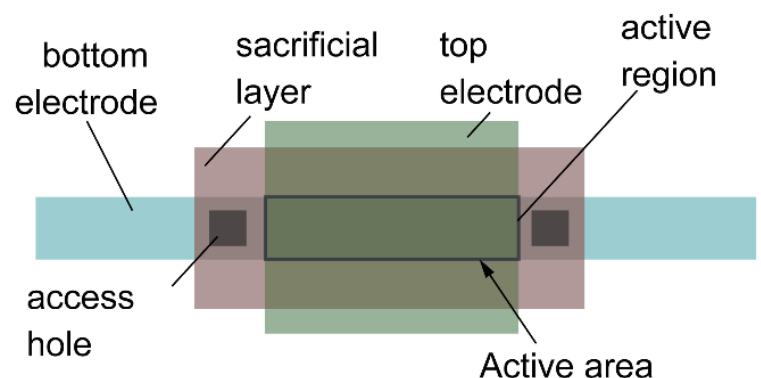

cross sectional view

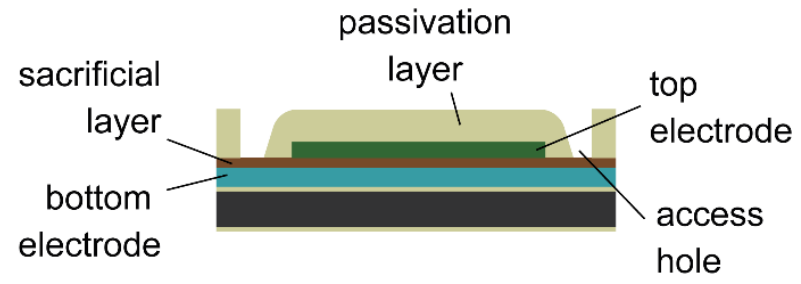

Figure A1. Top and cross sectional view of the device. The overlapping area of the top and bottom electrode represents the active area. This is where redox cycling occurs.

voltammetry was performed on this solution using a $10 \mu \mathrm{m}$ diameter platinum ultramicroelectrode (BASi, MF-2005), as shown in Figure A2 (blue curve). The sigmoidal oxidation wave confirmed that the $\mathrm{Fc}(\mathrm{MeOH})_{2}$ was predominantly in the reduced form. A small amount of reducing current was also observed, presumably due to some contamination and consistent with other reports in the literature. ${ }^{1}$ The crossing potential $\left(V_{r p}\right)$ was found to be $0.17 \mathrm{~V}$.

A small volume of solution (ca. $80 \mu \mathrm{l}$ ) was then introduced in a Polydimethylsiloxane (PDMS) reservoir in contact with a nanogap sensor, as per standard measurement protocol. Prior to this, both the device and the reservoir had been exposed to a chromium etchant solution (BASF, Selectipur) which was used to etch the sacrificial layer of the nanochannel. Sulphuric acid had similarly been used to clean the electrodes. It is likely that residues from both the etchant and the acid were absorbed within the PDMS. After conducting the measurements with the nanogap device, the 
solution was extracted out of the reservoir and cyclic voltammetry was performed again using the ultramicroelectrode, as shown in Figure A2 (red curve). The voltammogram exhibits a much more pronounced reduction wave, leading to an increase in the value of the $V_{r p}$ to $0.21 \mathrm{~V}$. This indicates that redox species in the solution became oxidized when introduced in the reservoir due to contamination, consistent with the measurements performed with the nanogap device.

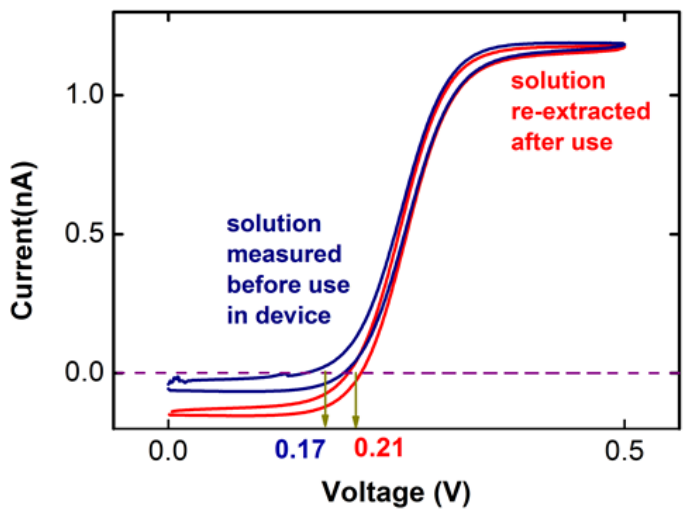

Figure A2. Cyclic voltammograms for $1 \mathrm{mM} \mathrm{Fc}(\mathrm{MeOH})_{2}$ in $0.1 \mathrm{M} \mathrm{KCl}$ before (blue curve) and after (red curve) being introduced in the nanodevice (scan rate $10 \mathrm{mV} / \mathrm{s}$ ).

\section{A.3 References}

1. J. W. Xiong and H. S. White, Journal of Electroanalytical Chemistry, 2013, 688, 354-359. 



\section{Appendix B}

\section{B.1 Experimental system}

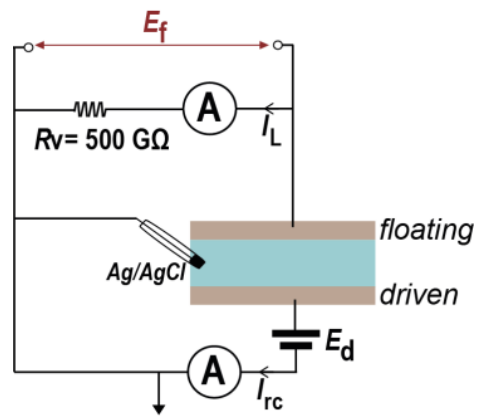

Figure B1. Schematic of experimental setup used. The floating electrode was connected to a high resistance $\left(R_{\mathrm{v}}=500 \mathrm{G} \Omega\right)$ and the current through it, $i$, was measured. The potential at the floating electrode, $E_{\mathrm{f}}\left(=R_{\mathrm{v}} i_{l}\right)$ was monitored while the bottom electrode, $E_{d}$, was driven to known potentials.

A conventional voltmeter has a high input impedance and therefore draws minimal current to measure the potential across two points. In practice, however, the value of this impedance is difficult to ascertain during a measurement as it may depend on 
parameters such as temperature or humidity level. In order to define a wellcontrolled environment suitable for quantitative analysis, we instead connected the floating electrode to a high-valued resistor $\left(R_{v}=500 \mathrm{G} \Omega\right)$. The leakage current through this resistor, $i$, was measured in order to monitor the floating potential, $E_{f}$ as shown in Figure B1.

\section{B.2 Estimation of the electrode capacitance}

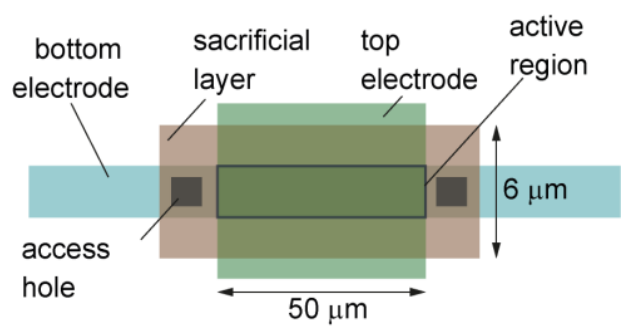

Figure B2. Schematic of the top view of the devices used (Type 1 and 2). The overlapping region between the top and bottom electrode shows the active region where redox cycling occurs and is estimated to be $150 \mu \mathrm{m}^{2}$. The area of the top and bottom electrode is the area that is exposed after the removal of the sacrificial layer which is estimated to be $300 \mu \mathrm{m}^{2}$ and $170 \mu \mathrm{m}^{2}$.

In theory, the ionic double layer capacitance, $C_{d}$, typically ranges from $10-40 \mu \mathrm{F} / \mathrm{cm}^{1}$ The area of the top floating electrode is $300 \mu \mathrm{m}^{2}$ (shown in Figure B2) and this yields a double layer capacitance, $C_{d, t}$, yields in the range of $15-60 \mathrm{pF}$. There is an additional capacitance, $C_{c}$, due to the conducting cables that are used for the measurement and contribute to about $100 \mathrm{pF} / \mathrm{m}$ (based on the type of cable used, 316/U). Furthermore, there exists a passivation capacitance, $C_{p}$, between the bulk solution and the electrodes across the protective passivation layer. This layer consists of $\mathrm{SiO}_{2} / \mathrm{SiN} / \mathrm{SiO}_{2}$ with thicknesses $90 \mathrm{~nm} / 325 \mathrm{~nm} / 90 \mathrm{~nm}$ and was deposited by PECVD. Based on the relative permittivity ( $\varepsilon=5$ and 7 for $\mathrm{SiO}_{2}$ and $\mathrm{SiN}$ respectively) and assuming that about $1300 \mu^{2}$ area of the electrodes was exposed to the bulk solution, the passivation capacitance, $C_{p}$, can be estimated to be $0.1 \mathrm{pF}$. However, in practice, the passivation layer and its capacitance can vary significantly from the expected values owing to the non-uniformities in the layer due to the method of deposition used in the microfabrication technique. 
The overall capacitance can be measured experimentally as well by monitoring the current at the driving electrode. At high enough concentrations, the driving potential is spontaneously tracked by the floating electrode. The capacitance can then be calculated from the measured current at the driving electrode when the driving electrode is cycled as shown in Figure B1. Capacitance was $\left(i_{p p} / 2 *\right.$ scan rate) found to be $590 \mathrm{pF}$. Thus the potential built up due to the double layer capacitance at top and bottom electrodes was $490 \mathrm{pF}$. However, the ratio of the area of the top electrode to that of the bottom electrode is 1.7 and therefore, the double layer capacitance, $C_{d}$, at the top electrode was $290 \mathrm{pF}$ and this value has been used for the simulations at the various concentrations.

a)

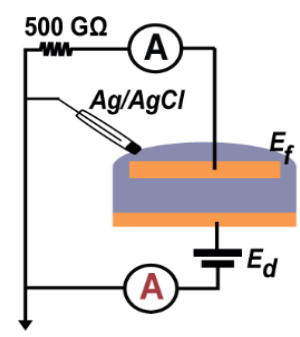

b)

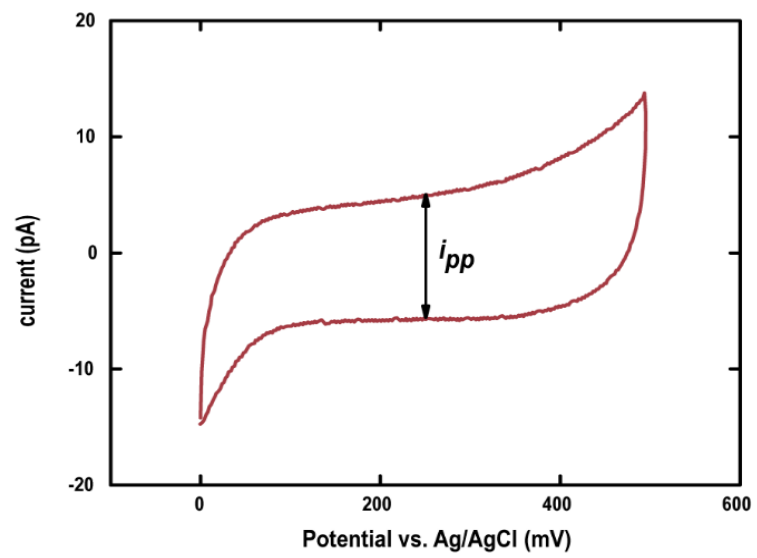

Figure B3. a) The experimental setup where the bottom electrode, $E_{d}$, was driven while the top electrode was left floating. b) Cyclic voltammogram of $250 \mu \mathrm{M}$ of $\mathrm{Fc}(\mathrm{MeOH})_{2}$ in $0.1 \mathrm{M}$ $\mathrm{KCl}$ aqueous solution at the driving electrode. Scan rate used was $10 \mathrm{mV} / \mathrm{s}$. Capacitance was calculated $\left(i_{p p} / 2\right.$ * scan rate) and was found to be $590 \mathrm{pF}$.

\section{B.4 Determination of root-mean-square potential}

To calculate the root-mean-square variation of the potential of the floating electrode, the average value of the blue curve was first subtracted and the root-mean-square value of the resulting curve, $V_{\text {rms }}$, was computed. Thus, any offset that might have been present in the raw data did not affect the calculation of the $V_{\text {rms }}$. 


\section{B.5 References}

1. Bard, A.J.; Faulkner, L.R., Electrochemical Methods: Fundamentals and Applications. Wiley: New York, USA, 2000. 


\section{Appendix C}

\section{C.1 Removal of physisorbed material}

After functionalization, the nanodevice was immersed in a beaker with MilliQ water which was vigorously stirred for at least $5 \mathrm{~min}$ in order to rinse all physisorbed

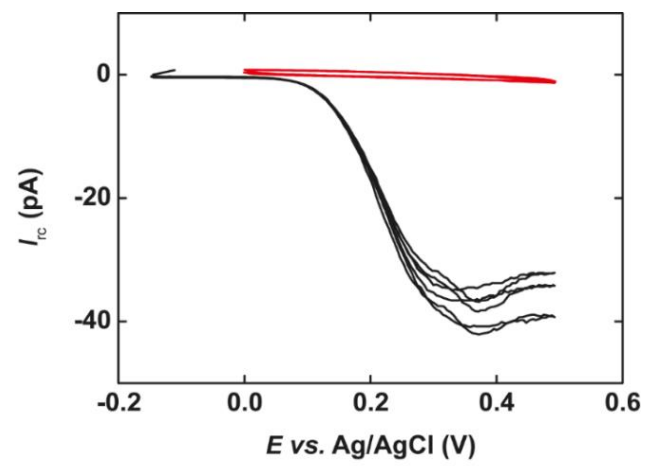

Figure C1. Redox cycling currents of a nanodevice before (black) and after (red) rinsing in water, after functionalization with $\mathrm{PEG}_{10 \mathrm{k}}$ and 2-mercaptoethanol instead of cystamine. Measurements were performed at a scan rate of $10 \mathrm{mV} / \mathrm{s}$. 
polymer chains from the device. To test whether this was sufficient, devices were treated with PEG10k in the same manner but with 2-mercaptoethanol instead of cystamine. Figure $\mathrm{C} 1$ shows the redox cycling current of a nanodevice after treatment with $\mathrm{PEG}_{10 \mathrm{k}}$ before (black) and after (red) rinsing in MilliQ water in the latter case. Here the current decreased to a purely capacitive signal, showing that polymers not specifically bound to the electrode surfaces were successfully eliminated from the device.

\section{C.2 Assessment of electrode surface roughness}

Devices were functionalized overnight with $2 \mathrm{mM}$ 11-(ferrocenyl)-undecanethiol in ethanol. Due to the high surface densities and suppression of the capacity by the long alkyl chains, surface densities could be determined for the top and bottom electrodes separately. The surface densities of the top and bottom electrodes were calculated as $\Gamma=1.9$ and $0.59 \mathrm{nmol} / \mathrm{cm}^{2}$, respectively. Given that the surface density of a full ferrocene layer is $0.45 \mathrm{nmol} / \mathrm{cm}^{2},{ }^{1}$ and assuming that both the electrodes are similarly functionalized, the surface densities thus determined imply a higher surface roughness for the top electrode than the bottom (4.2 and 1.3, respectively, for this particular device).

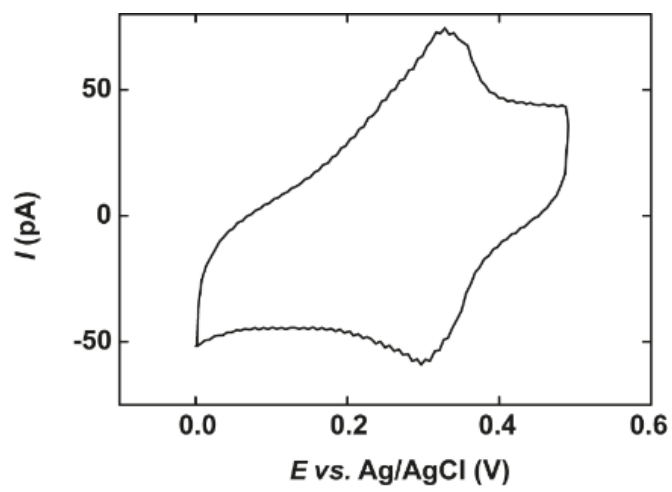

Figure C2. Cyclic voltammogram of 11-(ferrocenyl)undecanethiol functionalized electrodes measured at both electrodes simultaneously in a $1 \mathrm{M} \mathrm{NaClO}_{4}$ solution (scan rate $30 \mathrm{mV} / \mathrm{s}$ ). 


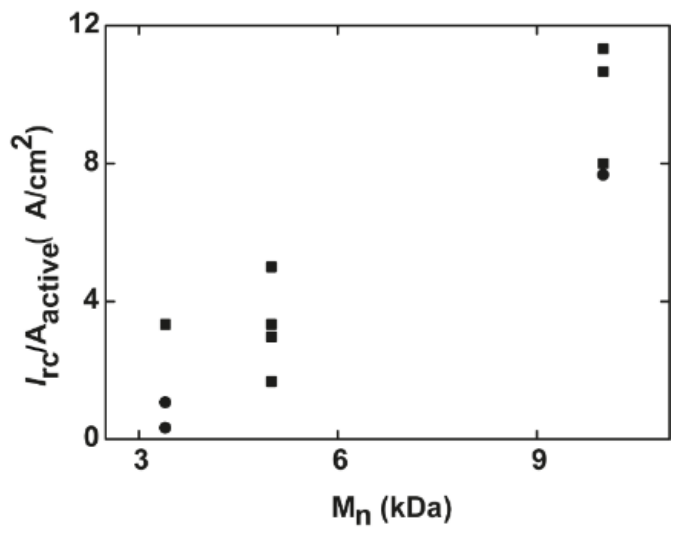

Figure C3. Redox cycling currents normalized to the active surface area. The measurements were performed on both types of devices, Type I = $\mathbf{\square}$, Type $\|=\bullet$

\section{C.3 Synthesis}

2-Ferrocene-ethylamine. 2-Ferrocene-ethylamine was synthesized by reduction of ferrocenylacetonitrile by $\mathrm{LiAlH}_{4}$ as described in literature, ${ }^{2}$ with some modifications. An amount of $0.25 \mathrm{~g}(6.5 \mathrm{mmol}) \mathrm{LiAlH}_{4}$ and $0.6 \mathrm{~g}(4.5 \mathrm{mmol}) \mathrm{AlCl}_{3}$ were carefully added to $10 \mathrm{~mL}$ dry THF while stirring in an ice bath. Ferrocene acetonitrile $(0.5 \mathrm{~g}$, $2.25 \mathrm{mmol}$ ) was dissolved in $5 \mathrm{~mL}$ dry THF and subsequently added to the cooled mixture and refluxed overnight under an argon atmosphere. After cooling, water was added drop wise to decompose the excess $\mathrm{LiAlH}_{4} .0 .25 \mathrm{~mL}$ of concentrated $\mathrm{NaOH}$ was added to destroy the formed $\mathrm{AlCl}_{3} / 2$-ferrocene-ethylamine complex. The aqueous phase was extracted three times with diethylether. The combined organic phases were dried with $\mathrm{MgSO} 4$ and filtered, and the solvent was removed by rotary evaporation. The product was purified by column chromatography with dichloromethane as the eluent. After drying in vacuum S5 a brown solid was obtained (0.13 g; 26\%). $1 \mathrm{H} \mathrm{NMR} \mathrm{(300} \mathrm{MHz,} \mathrm{CDCl} 3$ ): $\delta(p p m) 4.2(\mathrm{~m}, 9 \mathrm{H}, \mathrm{Fc}), 2.82$ (t, 2H, $\mathrm{CH}_{2}-\mathrm{Fc}$ ), $2.48\left(\mathrm{t}, 2 \mathrm{H}, \mathrm{CH}_{2}-\mathrm{N}\right)$.

NHS-PEG $3 k-F c$, NHS-PEG $5 k-F c$, and NHS-PEG ${ }_{10 k}-F c$. The NHS-PEG $-F c$ compounds were synthesized as described in literature. ${ }^{3}$ 2-Ferrocene-ethylamine $(6.9 \mathrm{mg}, 0.03 \mathrm{mmol})$ and $0.024 \mathrm{mmol}$ of a bis-NHS-functionalized PEG (PEG $3 \mathrm{k}^{-}(\mathrm{NHS})_{2}: 82 \mathrm{mg}$; $\mathrm{PEG}_{5 \mathrm{k}}-(\mathrm{NHS})_{2}$ : $120 \mathrm{mg}$; $\left.\mathrm{PEG}_{10 \mathrm{k}}-(\mathrm{NHS})_{2}: 250 \mathrm{mg}\right)$ were dissolved in $1 \mathrm{~mL}$ chloroform and $2 \mu \mathrm{L}$ triethylamine and stirred over night at room temperature under an argon 
atmosphere. The solvents were removed by rotary evaporation and the product was purified by size exclusion chromatography (Biobeads SX-1) with DCM as eluent. After drying in vacuo a brown solid was obtained.

Macro electrode functionalization. The Fc-PEG surface densities at different incubation times were measured on macro electrodes $(2 \mathrm{~mm}$ diameter gold disc electrodes; $\mathrm{CH}$ Instruments). Before modification the electrodes were polished using $50 \mathrm{~nm}$ alumina particles ( $\mathrm{CH}$ Instruments), followed by extensive rinsing with ethanol and 5 min of ultrasonic treatment in ethanol and $5 \mathrm{~min}$ in milliQ water. Subsequently the electrodes were cleaned electrochemically in $0.5 \mathrm{M} \mathrm{H}_{2} \mathrm{SO}_{4}$ by applying an oxidizing potential of $2 \mathrm{~V}$ for $5 \mathrm{~s}$ followed by a reducing potential of $-0.35 \mathrm{~V}$ for $10 \mathrm{~s}$. Then the electrode potential was scanned from $-0.25 \mathrm{~V}$ to $1.55 \mathrm{~V}$ and back for 40 cycles at a scan rate of $100 \mathrm{mV} / \mathrm{s}$. After cleaning the electrodes were rinsed with milliQ water and ethanol and dried under a flow of $\mathrm{N}_{2}$ and placed immediately in a 2 $\mathrm{mM}$ cystamine solution and left overnight. The surface densities of PEG were varied by changing the incubation time with a $0.2 \mathrm{mM}$ PEG solution and determined from by cyclic voltammograms measured at scan rates between 50 and $100 \mathrm{mV} / \mathrm{s}$, using a $\mathrm{CH}$ instruments bipotentiostat 760D, with $1 \mathrm{M} \mathrm{NaClO}_{4}$ as electrolyte vs. a $\mathrm{Ag} / \mathrm{AgCl}$ reference electrode and a platinum counter electrode.

\section{C.4 References}

1. Auletta, T.; van Veggel, F.C.J.M.; Reinhoudt, D.N., Self-assembled monolayers on gold of ferrocene-terminated Thiols and hydroxyalkanethiols. Langmuir 2002, 18, 1288-1293.

2. Seiwert, B.; Karst, U., Analysis of cysteine-containing proteins using precolumn derivatization with $\mathrm{N}$-(2-ferroceneethyl)maleimide and liquid chromatography/electrochemistry/mass spectrometry. Anal Bioanal Chem 2007, 388, 1633-1642.

3. Anne, A.; Moiroux, J., Quantitative characterization of the flexibility of poly(ethylene glycol) chains attached to a glassy carbon electrode. Macromolecules 1999, 32, 5829-5835. 


\section{Samenvatting}

In dit proefschrift worden de onderzoeksresultaten gepresenteerd welke uiteindelijk tot een platform moeten leiden bestaand uit een enkele chip ter bepaling van DNA sequenties via elektrische detectie. We hebben daartoe voor elektrochemische sensoren gekozen welke bijzonder geschikt zijn voor miniaturisatie en tegelijkertijd geïntegreerd kunnen worden met micro-elektronica. De sensor in dit specifieke geval is gebaseerd op cyclische redox reacties (redox cycling) welke plaatsvinden tussen twee elektrodes tientallen $\mathrm{nm}$ van elkaar gescheiden (nanokanaal). Doordat deze geminiaturiseerde sensoren elektrisch van aard zijn, kunnen ze goed toegepast worden in grootschalige microfabricage technieken. Ze kunnen gemakkelijk geïntegreerd worden met micro elektronica waarmee een kosten effectief bioelektronisch platform gerealiseerd kan worden met toepassingen van klinische observaties tot milieu analyses. Het detecteren van een enkel molecuul met specifieke eigenschappen welke als label gebruikt kunnen worden in DNA sequentie analyse is gedemonstreerd. In dit proefschrift zullen de verschillende ingrediënten voor zo'n geïntegreerd systeem besproken worden.

Het proefschrift start met een introductie van DNA sequentie analyse in hoofdstuk 1. Uitdagingen en nadelen van huidige methoden worden besproken. Daarna zullen eigenschappen van derde generatie technieken en een schematisch overzicht van het voorgestelde elektrochemische detectie platform gegeven worden. Ook zullen verschillende ingrediënten besproken worden welke tot de realisatie van het platform moeten leiden.

In hoofdstuk 2 worden concepten geïntroduceerd welke relevant zijn voor wetenschappers voor het opzetten van geminiaturiseerde elektrochemische platformen. Het verkleinen van zulke sensoren tot $\mathrm{l} \mathrm{m} / \mathrm{nm}$ afmetingen leidt vaak tot afwijkingen van het ideaal beeld (non idealities), maar kunnen tegelijkertijd bijdragen aan nieuwe of verbeterde detectiemethoden. De complexiteit van deze non idealities 
is hoog en vaak zijn ze moeilijk te identificeren. We bespreken de meest voorkomende problemen en de mogelijke oplossingen.

In hoofdstuk 3 verkennen we langs experimentele en theoretische weg nanokanaal sensoren waarbij de referentie electrode ontbreekt. De verschuiving van het vloeistofpotentiaal veroorzaakt door afwezigheid van de referentie electrode kan wanneer goed geïnterpreteerd verwerkt worden in de metingen.

Voor real-time DNA sequentie analyse is het gewenst dat het uitlees mechanisme zich op de chip zelf bevindt. In hoofdstuk 4 wordt een potentiometrische detectiemethode gebaseerd op redox cycling geïntroduceerd voor de detectie van sub-nM concentraties. De metal-oxide-semiconductor (CMOS) technologie, de basis voor een groot deel van de hedendaagse elektronica, is bijzonder geschikt deze methode op een eenvoudige manier te realiseren. Deze detectiemethode levert signalen die gemakkelijk verwerkt kunnen worden. Dit zou kunnen bijdragen aan de vereenvoudiging van de uitleescircuits.

Tot zover hebben we verschillende detectiemethoden onderzocht welke gebaseerd zijn op non idealities die ontstaan door het naar beneden schalen van sensor afmetingen. In hoofdstuk 5 concentreren we ons op de integratie van controle op de vloeistofstromen bij de fabricage van nanokanaal sensoren. De integratie bied een snelle methode voor het uitwisselen van vloeistof samples en minimaliseert tegelijkertijd de kans op verontreiniging. Er zijn vele toepassingen voor dit soort nanokanaal sensoren met geïntegreerde controle over de vloeistofstromen. Als demonstratie kiezen we voor dopamine hydrochloride (een middel waarvan bekend is dat het de electroden vervuilt) bij verschillende stroomsnelheden. De metingen bevestigen dat bij hogere stroomsnelheden de gevoeligheid van de nanosensoren toeneemt door de lagere vervuilingsgraad aan de electrode oppervlakten.

Ten slotte hebben we in hoofdstuk 6 laten zien dat PEG polymeer ketens (met redox actieve eindgroepen) verbonden met het oppervlak van een van de electroden elektronen kunnen uitwisselen wanneer ze aan redox cycling onderhevig zijn. Dit fenomeen hangt af van de ketenlengte die het mogelijk maakt dat het redox actieve deel heen en weer kan bewegen tussen de elektrodes. Echter, er is vastgesteld dat de gemeten stroomsterktes afhankelijk zijn van de pakkingsdichtheid van polymeerketens aan het electrode oppervlak. 


\section{Summary}

In this thesis, the results obtained from a research project aimed at ultimately realizing an all-electrical DNA sequencing platform onto a single chip are presented. To this end, we chose electrochemical sensors that are well suited for miniaturization as well as integration with microelectronics. In particular, our device involves redox cycling within a nanospaced twin electrode system (or nanogap). By virtue of being electrical in nature, such miniaturized sensors are well suited for large-scale microfabrication techniques. This allows them to be well integrated with microelectronics that can be used as a cost effective bio-electronic platform and can be utilized for a variety of applications from clinical examination to environmental analysis. Redox cycling at nanogaps have already demonstrated their ability to resolve single molecules with specific signatures that can be used as labels during DNA sequencing. In this thesis, we explore the individual ingredients for such an integrated system.

In the opening part of the thesis, Chapter 1, a brief introduction to DNA sequencing techniques is presented. The key challenges and disadvantages of present-day methods are discussed. Thereafter, the features of the third-generation techniques are introduced along with an overall scheme of the proposed electrochemical detection platform. The various ingredients for realizing this platform are also discussed.

In Chapter 2, the thesis goes on to introduce several concepts that are relevant to researchers while building a miniaturized electrochemical platform. This is especially relevant as scaling down such sensors to micro/nano domains often gives rise to non-idealities that are complex and quite challenging to identify. We address the most commonly encountered problems, and their possible alternate solutions.

In Chapter 3, we experimentally and theoretically explore detection within the nanogap sensors in the absence of the reference electrode. The shift in the solution 
potential caused by the absence of reference, when understood quantitatively, can be applied for accurate measurements.

In order to achieve real-time DNA sequencing, on-chip implementation of the readout mechanism is desirable. In Chapter 4, an alternate redox-cycling based potentiometric detection method is introduced to detect sub-nanomolar concentrations. The proposed method is easily realizable using complementary metal-oxide-semiconductor (CMOS) technology, that forms the basis for the vast majority of modern digital electronics. Such a detection scheme, if employed, would be able to deliver practical values of detection signals which could simplify the circuitry for read-out mechanism.

Up to this point, we explored the various detection schemes based on the nonidealities that arise due to scaling down of the device. In Chapter 5, we discuss the fabrication of such nanogap sensors focusing on the integration of fluidic control that not only offers a fast method for exchanging sample solution within the sensor but also minimizes the risk of contamination. To demonstrate one of the many utilities of these flow-incorporated nanogap sensors, we chose dopamine hydrochloride (a wellknown fouling agent) at various flow velocities. It is established that high flow rates within the nanogaps improve the sensitivity of these sensors by providing resistance to fouling of the electrode surfaces.

Finally, in Chapter 6, we have shown that surface-tethered PEG polymer chains with redox-active end groups allow the transfer of electrons between the two electrodes in nanogap devices as they undergo redox cycling. This phenomenon is dependent on the chain length of the polymer that allows the redox-active site to shuttle between the two electrodes facilitating electron transfer. It is observed, however, that the measured cycling currents are dependent on the surface density of the attached chains on the electrodes, which is lower for longer polymers. 


\section{Publications}

\section{Published}

1. Klaus Mathwig, Hamid R. Zefarani, J. Mathhäus Speck, Sahana Sarkar, Heinrich Lang, Serge G. Lemay, Liza Rassaei, Oliver G. Schmidt, Potential-Dependent Stochastic Amperometry of Multiferrocenylthiophenes in an Electrochemical Nanogap Transducer (J. Phys. Chem. C, DOI: 10.1021/acs.jpcc.6b07320).

2. Sahana Sarkar, Stanley C. S. Lai and Serge G. Lemay, Unconventional electrochemistry in micro-/nano fluidic systems, Micromachines, 2016, 7(5), 81.

3. Sahana Sarkar, Ab F. Nieuwenhuis, Shuo Kang, Klaus Mathwig and Serge G. Lemay, Integrated Microfluidics of Electrochemical Nanogap Sensors, Proceedings of the 19th International Conference on Miniaturized Systems for Chemistry and Life Science, Gyeongju, Korea, Oct. 25 - 29 (2015) 1522.

4. Sahana Sarkar, Klaus Mathwig, Shuo Kang, Ab F. Nieuwenhuis and Serge G. Lemay, Electrochemical Nanofluidic Assays in the Absence of Reference Electrode, Proceedings of the 18th International Conference on Miniaturized Systems for Chemistry and Life Science, San Antonio, USA, Oct. 26 - 30 (2014) 2122.

5. Sahana Sarkar, Klaus Mathwig, Shuo Kang, Ab F. Nieuwenhuis and Serge G. Lemay, Redox Cycling without Reference Electrodes, Analyst, 2014, 139, 60526057.

\section{$\underline{\text { Submitted }}$}

6. Tom Steentjes*, Sahana Sarkar*, Pascal Jonkheijm, Serge G. Lemay and Jurriaan Huskens, Electron transfer mediated by surface-tethered redox groups in nanofluidic devices (currently under review, * equal contribution). 
$\underline{\text { In preperation }}$

7. Sahana Sarkar, Ab F. Nieuwenhuis and Serge G. Lemay, Potentiometry at nanogaps at ultra-low concentration detection.

8. Sahana Sarkar and Serge G. Lemay, Integrated Microfluidics of Electrochemical Nanogap Sensors. 


\section{Acknowledgements}

On looking back, at the end of this journey that I had started alone, I find, instead, that I have been joined by many others, in one way or another. I wish to express my warmest gratitude to everyone who not only supported and encouraged me but also commented, criticized and questioned my decisions, both personally and academically, and have left a mark on this work.

Foremost, I would like to thank my advisor, Prof. Serge G. Lemay, for his excellent supervision, undisputed scientific strength and contribution to this thesis. Serge, thank you for giving me the opportunity to work under your guidance in this crucial phase of my education. I was a novice when I joined the group but you patiently taught me and encouraged me, especially in the initial phase of my Ph.D. I appreciate the freedom you gave me at work while steering me towards the right direction whenever needed. You always had time to listen and advice on roadblocks - major and minor- scientific and about managing life and people. Your positivity, ability to inspire and communicate with people with such ease are the most striking aspects of your personality, that I greatly admire and wish to inculcate in myself. I am really thankful for your supervision and am truly indebted to you.

I would also like to thank my previous advisors, Prof. J. Korvink and Prof. H. Reinecke, who gave me the opportunity to work and explore the field of MEMS and have continued to support and encourage me.

Prof. Jurriaan Huskens, your input and your suggestions regarding the final experiments were instrumental for the completion of Chapter 6, of this research work, for which I am very thankful to you.

Working in the group of Nanoioncs was quite a memorable experience both at work and outside. I would like to acknowledge some of the former members of our group who helped me in the initial days. Klaus, you supported me and got me started with my first experiments. Despite the fact, that you had physically left the group, email discussions regarding work continued. You have also given me sound advice on my professional future steps and I would like to thank you for your input. Shuo, I joined 
the group at a time when you were completing your thesis. Despite your busy schedule, you spent a lot of time training me in the clean room. Thank you. Dileep, even though we didn't work directly, you always lent a helping hand, while I had doubts. Liza, I met you only recently and you left me very encouraged and energetic after our conversations.

James, we have had some very exciting conversations/debates in these past years, which were quite stimulating and mostly fun to have. Stanley, your time in the group was both memorable and exciting. I have spent a lot of time scribbling on your board mostly about electrochemistry and occasionally about missing ingredients for the next borrel. Together, the both of you have guided me at work and given me valuable input in seminars, group discussions and book clubs that I really appreciate.

$A b$, you have been an absolute pleasure to work with. Whether it was a question about Dutch translations, or the setup, I have run into your office several times and you always had an answer with a smile on your face. I also had the pleasure of working with you and I am glad that you pushed the potentiometric method, which seemed quite impossible at the beginning and without your backing Chapter 4 of this thesis work would not have been possible.

Ingrid, you are the lifeline of our group and you gladly helped me with administrative questions, even before I had joined the group. Thank you.

Cecilia, Jin, Jos, Zinaida, Judith, Minmin, Monchai, and Chris together we covered most of the world in these four offices. The environment you all created was not only pleasant to work in but we also covered a multitude of other activities. Our regular coffee time/ lunch-table/fussball discussions ( and debates) were not only limited to work but covered myriads of topics- short and long- serious and silly. My time in the group was extremely memorable. Amongst the conferences, regular borrels, fussball, paintball, laser tag, curling, escape room, tree-to-tree climbing, international dinners, trips, and many others, my favorite was the traditional Nanoionics Christmas dinner. A magnificent and yet intimate candle lit dinner, accompanied by loud laughter and conversations complemented with festive Christmas songs in the background wrapped together with a pinch of suspense - anticipating the winner of the "best song of the year" contest.

Moving on, my work involved a fair amount of fabrication and several processes were quite new to me. This required guidance from several members in the Mesa+ cleanroom, who were always ready to have a quick discussion about the machine, process or simply in terms of technical support. Samantha, Hans, Rene, Huib, Ton, Marion, Mark, Henk and Peter, thank you. Meint, you took a final look at my design and improved it with your comments, which was very helpful in the overall fabrication. 
I also want to acknowledge the group of BIOS, where I often ran to use an equipment to do my final step of fabrication. The people in charge of this were always friendly and happy to help. I want to especially thank the technical support in the group who offered their help on several occasions. Johan, you took out time from your busy schedule and corrected my first set of masks and gave me valuable feedback both inside and outside the clean-room that I greatly appreciate. Jan, you also had several tips for me regarding the fluidic components.

Sip, you realized the substrate holder and made several changes to it (until I was absolutely happy with it) and had a final design ready within days.

Tom, we collaborated and did several measurements together and it was a pleasure to work with you. Apart from work, I also enjoyed discussing music with you.

I also had the privilege to guide and work together with Bram and Wouter, two very inquisitive and smart students, who put in a lot of effort into the preliminary measurements. I would like to thank you for your commitment and acknowledge your involvement in the project which ended up in Chapter 4 of my thesis.

The past four years weren't all about work and I was lucky to have distraction in the form of friends and family. Himanshu and Anirban, what started as a few neighbors cooking together quickly turned into a close-knit sky-box family. We have spent some fun and memorable times together. But above all, I could always count on you two for good home-cooked food, sometimes even accommodating my odd hours of measurement. You both have proceeded and taken the next step in your careers and I wish you both a happy and successful future.

The next pit stop consists of the group of theoreticians. Ahuja, you were one of my first and closest friends here. Some of my most memorable days here in Twente were spent with you. Together we have covered all forms of matter, you have made me derive equations on bare walls, in air, in water, and on snow (except with a pen on paper!). Kriti, I have spent a substantial amount of my time outside work with you. Together we have built up some beautiful memories (and some silly ones too) and I cherish each and every one of them. Metri, your regular "Pravachans" on Krishnamurthy to monkeys were equally fun and intriguing, especially after a couple of drinks. Finally, Csaba! Most of my days ended with long and pointless (occasionally morbid) arguments with you about Hitchens to duck shaped horses (or something ridiculous of that order). Your absolute randomness made me forget my worries and filled most of my days with uninhibited laughter. You were caring in your own unique way and you helped me keep my calm during my final days of writing this thesis. Thank you for being weirdly wonderful! 
I am also grateful to the AADHAAR fraternity, your sincere and earnest attempt to bring about a change is very inspiring. I enjoyed every bit of the meetings, marathons, event nights, videos and most importantly home-cooked potluck dinners. Starting with the heads of this family, Promodji and Vishakhaji, your wonderful hospitality not only during the meetings but especially during the festivals, far from home, were greatly appreciated. Thank you. Mitava, Poorvi, Harshita, Naveen, Shantanu, Manas, Kartikeya, Deva and Vishal, it was a pleasure to meet you and share such lovely experiences with you all.

Riza and occasionally Jasper and Sourish, I really enjoyed playing music with you all. Singing with you on the weekends would help me slow down and unwind for the beginning of the next week.

I also want to thank Subhajit, who was not only a part of my initial days here but also encouraged me and gave me the clarity I needed to take the step forward to start here. Thank you.

I want to address some of my close friends, who despite the distance, were a part of this journey. Shweta, Steffi, Ritika, Divya, Ritesh, and Bhavesh, either during my holidays or on long skype/phone calls, time was well spent with you all and it helped me unfold in many different ways. Apu mashi, Nobho Mesho and Ahana you were my family here in Europe. Thank you for your love and support over the past few years. My Family, the De s and Sarkar s, I greatly appreciate your constant encouragement and crazy yet entertaining conversations. Dadu and Thama, Kakima \& Kaka, I really appreciate your support and continual inspiration.

There were times when all I needed to hear was "you can do it, my boy!" Last but not the least, $\mathrm{Ma}$ and Baba, your constant support and encouragement each and every day provided me stability and gave me the confidence to keep pushing ahead both personally and professionally. Thank you for your love and support throughout my life. 
"All forms that perish other forms supply' (By turns we catch the vital breath and die) Like bubbles on the sea of matter borne' They rise' they break' and to that sea return."

Alexander Pope, An Essay on Man 



\section{About the author}

Sahana Sarkar was born on 3rd August 1987 at Purulia, West Bengal, India. She completed her Bachelor of Engineering in Instrumentation and Control Engineering in 2009 at the Manipal Academy of Higher Education (MAHE) in Manipal, Karnataka, India. In the summer of 2 nd year, at MAHE, she did a brief internship at the Gas Authority of India Ltd, (GAIL), at the Gas based petrochemical complex at Pata, UP, India. Thereafter, she did her final year project at the Yokogawa India Ltd, Bangalore, where she worked on the integrated control system for the Rajasthan Northern area Develpement (RNAD).

After graduation, she began her Masters in Microsystems engineering at IMTEK, University of Freiburg, Germany. In 2010, she worked towards the Development of liquid filled tunable lenses for retinal implants under the supervision of Prof. Jan Korvink. She continued here Masters thesis on the same project and modeled lens deflection analytically by using fundamental differential equations of non-linear plate deflection by applying the Zernike-Galerkin Method (ZGM). Thereafter, she worked under Prof. Holger Reinecke, designing a fast shutter mechanism based on magnetic actuation for a real time a 3D endoscopic system. After completion of her Masters degree from University of Freiburg, Germany, in 2012, she worked briefly at IHP Microelectronics, in Frankfurt am Oder, under Prof. Giovanni Capellini in the field of CMOS/BiCMOS compatible lasers, where she focused on modeled strain distribution on Germanium bridges sandwiched between SiN and Si.

Motivated to work in the field of nano-scaled fluidics, she began her PhD research work in the Dept. of Nano-lonics, University of Twente, Netherlands, where from November 2012, she started working in the field of Electrochemical Nanofluidic sensors, under the supervision of Prof. Serge Lemay. The results of her thesis results are presented in this Ph.D. dissertation. 


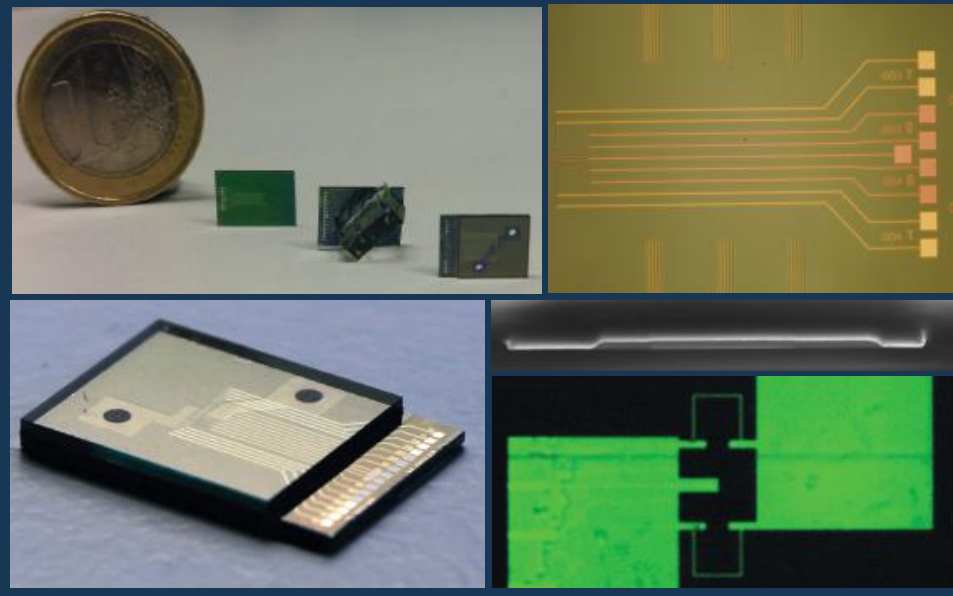

ISBN: 978-90-365-4241-8

DOI: $10.3990 / 1.9789036542418$ 